\title{
Methods to study organogenesis in decapod crustacean larvae Il: analysing cells and tissues
}

\author{
R. R. Melzer ${ }^{1,2,3}$, F. Spitzner ${ }^{4}$, Z. Šargač ${ }^{4}$, M. K. Hörnig ${ }^{4}$, J. Krieger ${ }^{4}$, C. Haug 2,3 , J.T. Haug ${ }^{2,3}$, T. Kirchhoff ${ }^{4}$, R. Meth ${ }^{4}$, \\ G. Torres ${ }^{5}$ and S. Harzsch ${ }^{4^{*}}$
}

\begin{abstract}
Cells and tissues form the bewildering diversity of crustacean larval organ systems which are necessary for these organisms to autonomously survive in the plankton. For the developmental biologist, decapod crustaceans provide the fascinating opportunity to analyse how the adult organism unfolds from organ Anlagen compressed into a miniature larva in the sub-millimetre range. This publication is the second part of our survey of methods to study organogenesis in decapod crustacean larvae. In a companion paper, we have already described the techniques for culturing larvae in the laboratory and dissecting and chemically fixing their tissues for histological analyses. Here, we review various classical and more modern imaging techniques suitable for analyses of eidonomy, anatomy, and morphogenetic changes within decapod larval development, and protocols including many tips and tricks for successful research are provided. The methods cover reflected-light-based methods, autofluorescence-based imaging, scanning electron microscopy, usage of specific fluorescence markers, classical histology (paraffin, semithin and ultrathin sectioning combined with light and electron microscopy), X-ray microscopy $(\mu \mathrm{CT})$, immunohistochemistry and usage of in vivo markers. For each method, we report our personal experience and give estimations of the method's research possibilities, the effort needed, costs and provide an outlook for future directions of research.
\end{abstract}

Keywords: EcoDevo, MorphoEvoDevo, Brachyura, Immunohistochemistry, Micro-computed tomography, Larval development, Electron microscopy, Organogenesis, Imaging techniques, Histology

\section{Background}

Whereas crustacean embryos are popular study objects of developmental biologists (recent reviews, e.g., [1-7]), analyses on organogenesis in crustacean larvae somewhat lag behind (see Table 1 in companion paper [8]) although, for many decades, brachyuran larvae have served as distinguished models in the field of Ecological Developmental Biology (reviews $[9,10])$. Larval development of decapod crustaceans embraces a wealth of fascinating ontogenetic

\footnotetext{
*Correspondence: steffen.harzsch@uni-greifswald.de

${ }^{4}$ Department of Cytology and Evolutionary Biology, Zoological Institute

and Museum, University of Greifswald, Greifswald, Germany

Full list of author information is available at the end of the article

We dedicate this review to Mireille and Guy Charmantier, two of the

pioneers in analysing organogenesis in decapod crustacean larvae
}

processes that are well worth analysing. Many decapod crustaceans display a complex life cycle which includes pelagic larvae and in many subgroups benthic juvenileadult stages so that these organisms undergo a distinct niche shift during the transition from larvae to the juvenile phase (reviews [9-14]). Larvae develop by successive moults through a species-dependent number of larval instars and, in the absence of parental care, are adapted to developing in the plankton concerning aspects such as movement, nutrition, and sensing (reviews [11, 12, 15]). Larvae also feature a rich behavioural repertoire which allows them to respond to variations in environmental key factors such as light, hydrostatic pressure, tidal currents, temperature, salinity, and food concentration (reviews [10, $16,17])$. In many decapod groups, after two metamorphic moults (reviews [18-21]), larvae eventually settle to the 
benthos where juvenile growth into adulthood, reproduction, embryonic development, and larval hatching occurs.

Cells and tissues form the bewildering diversity of larval organ systems which are necessary for autonomously surviving in the plankton ([11, 22-25]). As the larvae develop, their organs continue to grow in size and complexity. For the developmental biologist, decapod larvae provide the fascinating opportunity to observe a complete and complex organism on a single microscopic slide and the chance to analyse how the adult organism unfolds from organ Anlagen compressed into a miniature larva in the sub-millimetre range. Furthermore, several of the metamorphic processes are promising targets for future investigations by developmental biologists [22]. For example, the neuromuscular system of the pereiopods must be refined during the seemingly abrupt transition to functionality during the first metamorphic moult. Furthermore, as gas exchange switches from the branchiostegites in the Zoea to the gills in the Megalopa, the circulatory system must adapt to new routes of haemolymph flow. Contrary to holometabolous insects, for example, which in the pupal stage develop a cuticular armor to shield the developing organism against environmental impacts, such metamorphic transformations must occur "on the fly" in a planktonic larva that is constantly exposed to predation and must respond to abiotic environmental parameters.

This set of two papers sets out to summarise current techniques to analyse organogenesis in decapod crustacean larvae. In the companion paper [8], we have already described the techniques for culturing larvae in the laboratory and dissecting and chemically fixing their tissues for histological analyses. Here, we continue with describing state-of-the-art morphological and anatomical techniques that are established for various decapod larvae. We will begin our account by describing techniques to analyse the outer larval surface such as macrophotography, imaging of the cuticular autofluorescence and scanning electron microscopy. Such techniques are not only suited to analyse the ontogeny of the general larval morphology but also ontogenetic modification of specific organs such as sensory setae and the ommatidia of the compound eyes. We continue with techniques to study the anatomy of individual organs at the tissue and cellular levels such as classical histology, x-ray microscopy, immunohistochemistry and the use of cell proliferation markers.

\section{Methods to visualise the outer morphology Reflected-light-based methods: macro- and micro-photography Introduction}

Reflected-light-based methods in the context of macroand micro-photography (Fig. 1) allow the documentation of an animal's general outer morphology, but also very fine details on the outer surface. These methods demand a relatively small amount of equipment and involve low costs (Table 1), but provide high flexibility in the setup without any destructive manipulation of the specimen. Reflected-light-based methods are essential for studying the outer morphology of an animal, especially if true colour information is desirable. Providing a well-documented general impression of the animal is also useful if the outer morphology is not the focus of the study. This allows a non-expert reader to gain an overview of the general habitus in high quality. One main advantage is the high flexibility of the method as it can be applied using nearly all kinds of microscopes, but also macrophotographic setups. The limitation of magnification depends on the used optics. For generating consistently sharp high-resolution images, single images in $x^{-}, y^{-}$, and $\mathrm{z}$-axis can be combined with freely available or low-cost software.

\section{Description of the method}

Specimen preparation Fixed specimens (e.g., in ethanol) should be photographed directly in the fixative, otherwise delicate structures such as antennae or fine setae will agglutinate. These fluids of course should not contain particles that may obstruct the specimens. Small glass blocks can be used to adjust and fix the specimen in the desired orientation, and coverslips on top of the specimen (with glass blocks or plates as spacers to the bottom of the container and fixed with small weights such as screw nuts) will prevent movement. In principle, all kinds of containers such as Petri dishes or plastic containers filled with coloured silicone, e.g., in black or white are useful. If the specimen is very bright or dark, a black or white background can cause too low or too extreme contrasts. In these cases, it can be helpful to use a grey or blue background (the latter should be decolourised during image post-processing).

Setup Reflected-light-based imaging can be applied with nearly all types of microscopes or macro-setups. In all cases, light is one of the most important factors. Several light sources should be arranged around the specimen to avoid shadows, which may hide details or suggest false-positive details (e.g., [26]). To avoid reflections causing strong artefacts and for enhancing colour-contrast, cross-polarised light can be used. To that end, polarisation filters could be mounted in front of the light sources and in front of the objectives (e.g., [27]). If the polarisation filters are rotated about 90 degrees to each other, nearly all reflections should be obliterated.

Micro-photography A microscope equipped with a builtin camera and reflected light is useful (Fig. 1d), but not 

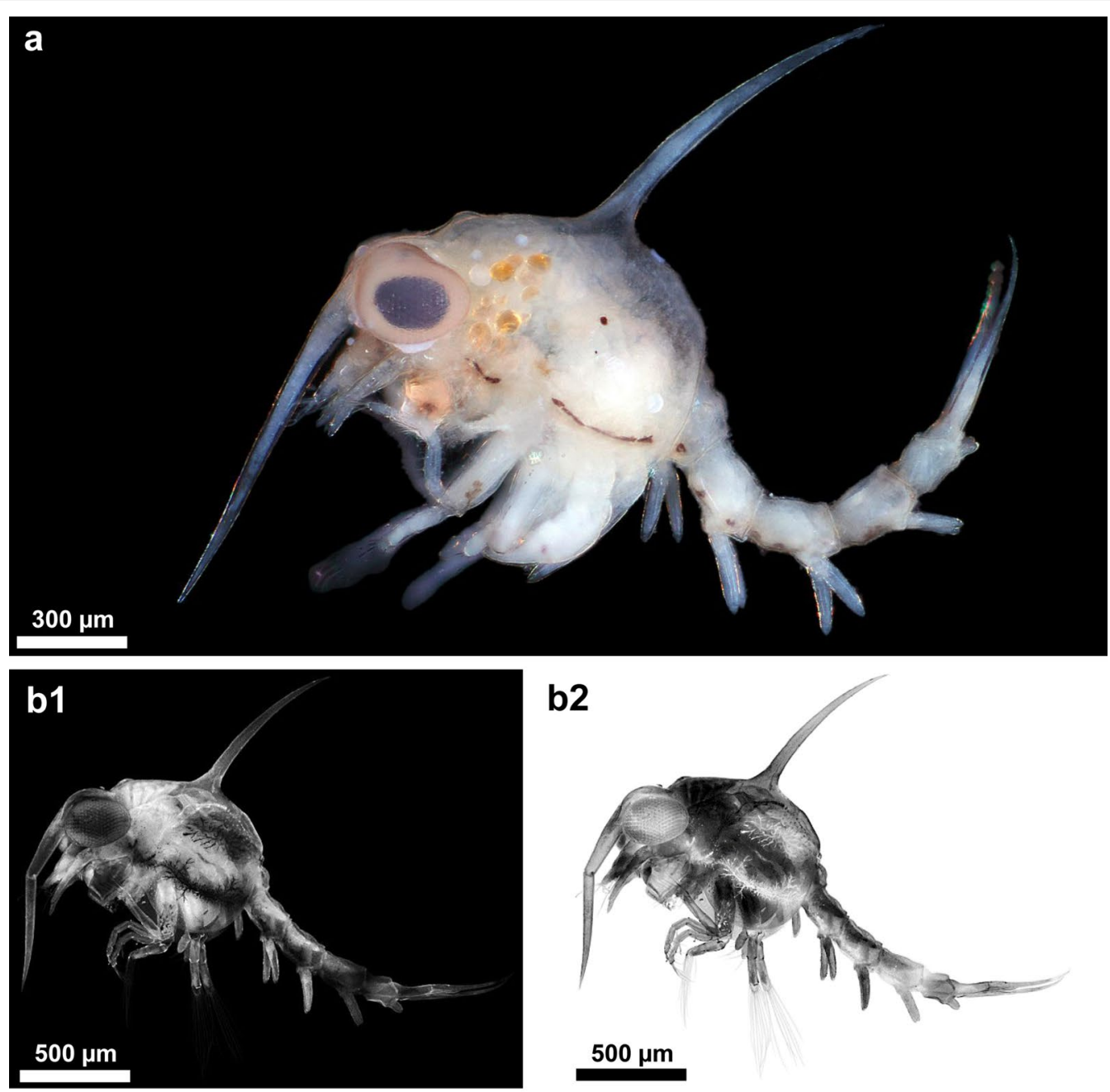

\section{$500 \mu \mathrm{m}$}
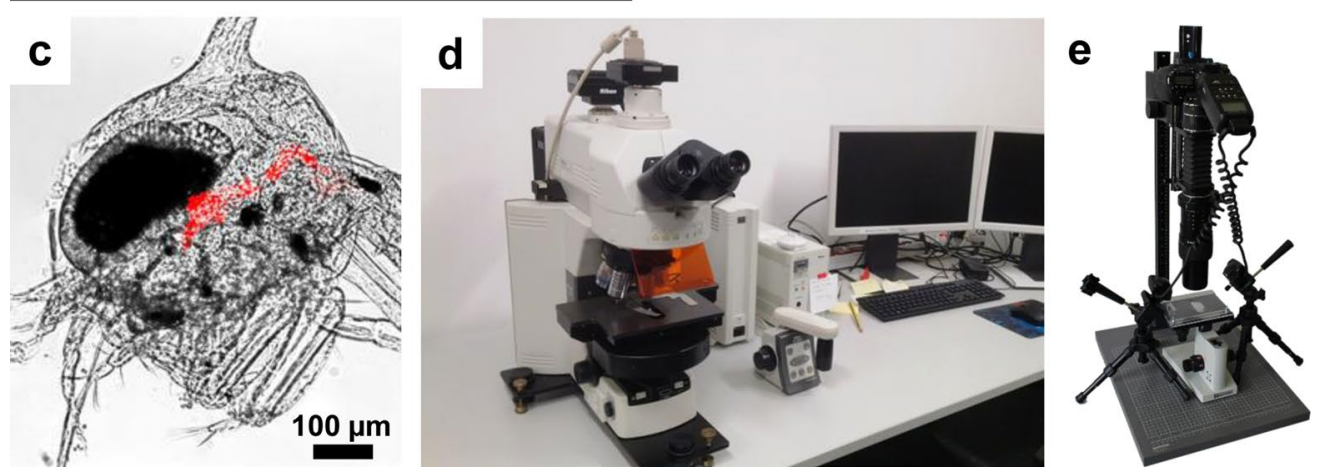

Fig. 1 Macro- and microphotography, and autofluorescence-based imaging of decapod larvae (see section "Reflected-light-based methods: macro- and micro-photography" and "Autofluorescence-based imaging"). a Macrophoto of a Carcinus maenas Zoea IV using the camera setup shown in panel e) (M. Hörnig, unpublished). b1, b2 Autofluorescence image of a Carcinus maenas Zoea IV mounted on a microscopic slide in ethanol and cover-slipped using modelling clay as spacers (F. Spitzner, unpublished). The image was taken using a Nikon Eclipse 90i upright microscope (excitation 359-371 nm; emission 379 nm; see panel $\mathbf{d}$ equipped with a digital camera. Image b2 was black-white inverted using image processing software. Note that more details of the setae on the exopods of the maxillipeds are visible. c Two-channel image of Zoea 1 of Xantho poressa combining brightfield image with chlorophyll autofluorescence in the intestine after feeding with diatoms (red) (modified from [33]). d Fully motorised upright fluorescent microscope Nikon Eclipse 90i (image S. Harzsch). e Macrophotographic setup (image M. Hörnig) 
Table 1 Summary of speed (from slow* to fast****) and costs (from rather cheap $€$ to rather expensive $€ € € €$ ) of the methods presented in this paper

\begin{tabular}{|c|c|c|c|c|}
\hline & Speed & $\begin{array}{l}\text { Costs of } \\
\text { reagents }\end{array}$ & $\begin{array}{l}\text { Costs of lab } \\
\text { instruments }\end{array}$ & $\begin{array}{l}\text { Costs of } \\
\text { imaging } \\
\text { equipment }\end{array}$ \\
\hline Macrophotography & $* * * *$ & $€$ & $€$ & $€$ \\
\hline $\begin{array}{l}\text { Autofluorescence } \\
\text { microscopy }\end{array}$ & $* * * *$ & $€$ & $€$ & $€ € / € € €$ \\
\hline SEM & $* *$ & $€ €$ & $€ €$ & $€ € € €$ \\
\hline Micro CT & $* * *$ & $€$ & $€ €$ & $€ € € €$ \\
\hline Paraplast histology & $* *$ & $€ €$ & $€ € €$ & $€ / € € €$ \\
\hline Semithin histology & $* *$ & $€ € €$ & $€ € €$ & $€ / € € €$ \\
\hline TEM & $*$ & $€ € €$ & $€ € € €$ & $€ € € €$ \\
\hline $\begin{array}{l}\text { Immunohistochem- } \\
\text { istry }\end{array}$ & $* *$ & $€ € € €$ & $€$ & $€ € / € € € €$ \\
\hline Proliferation markers & $* *$ & $€ € € €$ & $€$ & $€ € / € € € €$ \\
\hline Nuclear dyes & $* * * *$ & $€ €$ & $€$ & $€ € / € € € €$ \\
\hline
\end{tabular}

Some methods are much more time consuming than others: ${ }^{* * *}$ hours, ${ }^{* *}$ one day, ${ }^{* *}$ several days, ${ }^{*}$ several weeks. The costs of reagents summarises necessary chemicals: $€<10, € €<100$, €€€ $<500$, €€€€<1,000. The costs of lab instrumentation summarises standard equipment such as orbital shakers, ovens and microtomes: $€<500$, $€<5,000$, $€ €<10,000$, $€ € € €<20,000$. For the imaging equipment, some analysis is possible with rather simple microscopes, but more sophisticated instruments (such as a confocal laser scanning microscope) can be rather expensive: $€<2,000$, $€ €<20,000$, $€ €<100,000$, $€ € € €$ over 250,000.

necessary. For usual transmitted-light microscopes, several light sources, such as gooseneck lamps or even flashlights (making the system more mobile), each equipped with polarisation filters, can be arranged around the specimen for illumination. For capturing images, a simple ocular camera can be used (such as Scopetek DCM 510). It is unnecessary to use cameras with high resolution since this factor is mainly limited by the objective lenses and single images can be stitched together (see below).

Macro-photography There are several ways to build up a macro-setup. Here, we refer to a custom setup widely used by the authors (Fig. 1e). The specimen in Fig. 1a was photographed with a Canon 70D reflex camera equipped with a Canon MP-E $65 \mathrm{~mm} \mathrm{f} / 2.81-5 \times$ macro lens. To enhance the magnification beyond the maximum magnification of the objective, close-up rings are used (for example bridging a total of $86 \mathrm{~mm}$ distance in the case of Fig. 1a). Illumination is provided using a Canon MT24EX Macro Twin Lite. Flash heads are fixed to tripods and arranged on two opposite sides close to the specimen. Flashes and objective are equipped with polarisation filters for cross-polarised light. We use linear filter foils the brand of which does not matter, and we have even used polarization foils removed from old flatscreen monitors and this works well. The camera is mounted on a repro stand with an additional macro slider/focusing rail, with the lens pointing downwards. The additional macro slider allows a more precise adjustment of the camera position and can be useful to photograph a specimen from different angles, e.g., for generating stereo anaglyph images.

Composite images To enhance the field of view and generate consistently sharp images in high resolution, recordings of several single images in $z$ - and/or xy-axis can be taken and subsequently fused/stitched together ([28]; Table 2). For macro-setups, a table with fine drive in all axes (scanning stage) is highly recommended (e.g., dismounted from discarded microscopes). Z-image stacks can be fused to achieve consistently sharp images using free or low-cost software, such as ImageAnalyzer or Combine ZM/ZP. The fused images can subsequently be merged in $\mathrm{x}$ - and $\mathrm{y}$-axis, e.g., with Photoshop (Photomerge) or Microsoft ICE (Table 2).

\section{Additional comments}

Due to the high flexibility of a custom setup, it can be adapted for many approaches without any manipulation of the specimen and to variable specimen sizes. This is especially important for very rare collection material, as well as fossil specimens $([26,29-31])$. As in principle, not much equipment is needed (Table 1 ), it is relatively easy to build up transportable setups for, e.g., collection material that cannot be transported. Macro-setups can be tuned to magnifications in a range close to those of light microscopes (e.g., see Fig. 1a, photographed with a macro-setup) for specimens of even less than $1 \mathrm{~mm}$. Problems due to movements of specimens immersed in fluids result in strong artefacts in z-stacks. Hence, a proper mounting (without manipulation) of the specimen is necessary, but sometimes challenging. This holds also true for floating particles (dust) especially when working with high magnifications (here, filtering of the fixative can improve image quality). Even by using polarisation filters (and also diffusers), reflections cannot always be eliminated, which may cause strong artefacts, especially in fused z-stacks. This can be avoided by using fluorescence-based methods if the specimen exhibits autofluorescence or has been stained with a fluorescent dye $([27,32])$.

\section{Autofluorescence-based imaging Introduction}

The autofluorescence of the crustacean cuticle, without any staining, can be well used for documenting the outer morphology of a specimen (Figs. 1b1, b2, 2; [27, 32]). As compared to reflected-light-based methods, there are a number of differences, namely, that the contrast can be significantly enhanced, and that many fine details of the cuticle can be made visible (Fig. 2). In this aspect, 
Table 2 Selected examples for image processing software

\begin{tabular}{|c|c|c|c|}
\hline & Freely available & Operating system & Comments \\
\hline \multicolumn{4}{|l|}{ General image processing } \\
\hline Adobe Photoshop (Adobe Inc.) & & Microsoft Windows; macOS; Linux & \\
\hline CoreIDRAW (Corel Corporation) & & Microsoft Windows; macOS & \\
\hline FIJI/ImageJ & Yes & Microsoft Windows; macOS; Linux & $\begin{array}{l}\text { Open source, extremely variable in options, } \\
\text { various plugins available [153] }\end{array}$ \\
\hline GIMP & Yes & Microsoft Windows; macOS; Linux & Open source [157] \\
\hline \multicolumn{4}{|l|}{ Stacking (Z-Stacks) } \\
\hline Zerene Stacker (Zerene Systems LLC) & & Microsoft Windows; macOS; Linux & \\
\hline Image Analyzer (MeeSoft, Michael Vinter) & Yes & Microsoft Windows & MultiFocus plugin [158] \\
\hline Combine ZM/ZP (Alan Hadley) & Yes & Microsoft Windows & \\
\hline \multicolumn{4}{|l|}{ Stitching (X-/Y-AXIS) } \\
\hline Adobe Photoshop (Adobe INC.) (photomerge) & & Microsoft Windows; macOS; Linux & $\begin{array}{l}\text { Photomerge plugin "Interactive Layout" only } \\
\text { available up to CS5 }\end{array}$ \\
\hline Microsoft Image Composite Editor (ICE) & Yes & Microsoft Windows & \\
\hline \multicolumn{4}{|c|}{ 3D imaging/3D reconstruction ( $\mu \mathrm{CT}, \mathrm{CLSM}$, sectional image stacks) } \\
\hline $\begin{array}{l}\text { Amira (Zuse Institute Berlin; Thermo Fisher } \\
\text { Scientific) }\end{array}$ & & Microsoft Windows; macOS; Linux & \\
\hline Imaris (Bitplane) & & Microsoft Windows; macOS; & \\
\hline VGSTUDIO Max (Volume Graphics) & & Microsoft Windows; macOS; Linux & \\
\hline Drishti (Ajay Limaye) & Yes & Microsoft Windows; macOS; Linux & Open source [159] \\
\hline $\begin{array}{l}\text { SPIERS (Palaeoware; Mark Sutton, Russell Gar- } \\
\text { wood, Alan R.T. Spencer) }\end{array}$ & Yes & Microsoft Windows; macOS; Linux & Open source [160] \\
\hline $\begin{array}{l}\text { Microscopy Image Browser (MIB; Electron } \\
\text { Microscopy Unit, University of Helsinki) }\end{array}$ & Yes & Microsoft Windows; macOS; Linux & [161] \\
\hline Blender (Blender Foundation) & Yes & Microsoft Windows; macOS; Linux & $\begin{array}{l}\text { Open source; Postprocessing 3D Models, } \\
\text { Surface visualisation [162] }\end{array}$ \\
\hline Osirix (Pixmeo SARL) & Yes, in lite version & macOs & \\
\hline
\end{tabular}

it will allow access to morphological structures that are also well recognisable in scanning electron microscopic (SEM) images, but much faster and without the necessity of the work-intense, partly destructive methods. This allows, for example, to use this method for rare and historical specimens. Additionally, also internal structures can be made visible without staining the specimen, for example, muscles, parts of the nervous system, or the digestive system. Similar to reflected-light-based methods, composite imaging (Table 2) will be necessary to overcome limitations in depth of field and field of view. Often, the same principle setups as for reflected-light based methods can be used. This allows a combination of the methods into one image. In many cases, autofluorescence, for example, is not very good for documenting projecting setae. Therefore, the same structure can be documented in addition to transmitted-light methods for gathering details of the projecting setae (e.g., differential interference contrast (DIC), polarisation contrast, or darkfield; [32], their Fig. 4f).

Not only the crustacean cuticle shows autofluorescence, but also plant cells do. For example, chlorophyll autofluorescence has been used in analyses of feeding experiments. For about 15 to 20 years, it is known that early zoea stages, which for a long time were thought to be exclusively carnivorous, also feed on phytoplankton such as diatoms. Tanković et al. [33] have detected and visualised chlorophyll in the intestine of Zoeae with the fluorescence microscope and even quantified the number of diatoms. This is possible because early Zoeae are transparent. In their paper, the authors visualised and measured chlorophyll fluorescence in unfed (control) and fed Zoea 1 stages of Xantho poressa, and determined the number of diatoms ingested by the animals. That way, they also visualised the intestine of living larvae (Fig. 1c).

\section{Description of the method}

Setup As pointed out, most setups used for reflectedlight-based imaging can also be applied for autofluorescence-based methods, with nearly all types of microscopes or macrophotographic setups. The main important factor for achieving fluorescence is a reliable set of filters and a sufficient amount of light that is evenly distributed (as for reflected-light setups). For microscope setups, the 


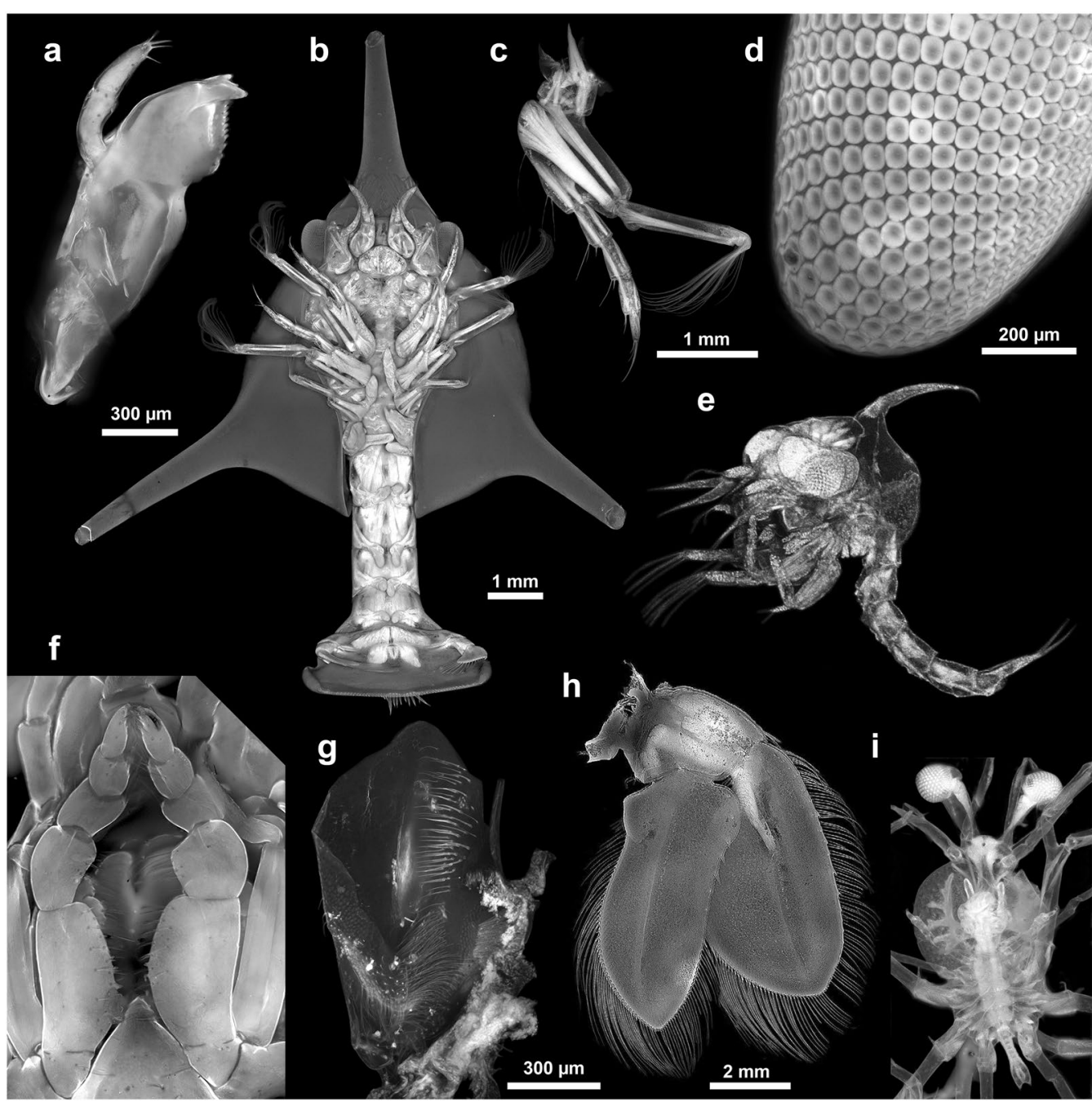

Fig. 2 Different examples of images of decapod crustacean larvae recorded with autofluorescence as described in section "Autofluorescence-based imaging" (images not to scale); epifluorescence images except for e). a Isolated mandible of a late larva of Homarus gammarus (modified from [163]). b Late Zoea of a false sand crab (Hippidae), ventral view (modified from [164]). c Maxilliped of late Zoea of a false sand crab (Hippidae; modified from [164]); note the well apparent muscles. $\mathbf{d}$ Compound eye of late Zoea of an anomalan crustacean (modified from [165]). e Zoea of an undetermined brachyuran crab, lateral view, confocal laser-scan microscopy (C. Haug, J.T. Haug, unpublished). f Mouthparts of a Megalopa of a brachyuran crab (C. Haug, J. T. Haug, unpublished). $\mathbf{g}$ Part of gizzard of an anomalan Zoea (modified from [165]). $\mathbf{h}$ Uropod of an Eryoneicus of a polychelidan lobster (modified from [30]). i Central body of Phyllosoma of an achelatan lobster; note the well apparent nervous system and midgut glands (C. Haug, J. T. Haug, unpublished)

simplest illumination is coaxial (built-in) fluorescence light. Yet, for microscopes that lack such a built-in option and for macro-setups, it is possible to simply use several gooseneck lamps or focusable torches as well as LEDs. The cheapest available filters are the coloured foils of anaglyph glasses. The most readily available ones will be red-cyan foils, but it will in principle work with any type of anaglyph glasses or in general complementary filters. The filter with the colour with the shorter wavelength (e.g., cyan) has to be placed in front of the light sources; the filter with the colour with the longer wavelength (e.g., red) has to be placed in front of the lens/objective. As complementary filters are used, the light directly reflected from the specimen will be blocked, only the newly emitted (fluorescence) light will pass the filter. Autofluorescence-based imaging requires longer exposure times due to the low intensity of the emitted light. Therefore, it is especially important that the specimen is properly fixed in place and will not move during the documentation. Sometimes it is necessary to document the specimen with different exposure times as some areas exhibit strong autofluorescence while others show rather weak autofluorescence. If only one exposure 
time is used, some areas will appear overexposed while others are barely visible. The images documented with different exposure times need to be combined during image processing. In addition, more complex setups with structured light can be used for recording images under autofluorescence. This includes the now more common confocal laser scanning microscopy (cLSM; [34]), but also microscopes with a spinning disc/Nipkow disc and potentially also other types such as light-sheet microscopes. The main advantage of all these setups for autofluorescence imaging is the reduction of scattered light.

Image processing The general processing of the recorded image stacks is the same as explained above for reflectedlight-based methods. However, the resulting composite images require some further post-processing (Table 2). Images documented with autofluorescence-based methods often have a colour tinge. This may either be the result of the colour of the filter in front of the lens/objective; or it may be the result of a colour artificially added via the software of the microscope. The latter is initially intended to show which dye has been used to stain the documented specimen, e.g., in histochemical studies. However, as the specimens are not stained for autofluorescence imaging, the colour is meaningless in this context. This is in general true for all cases in which only a single channel is used, which means if only the intensity of a single dye is shown. In these cases, the artificial colour makes the image unnecessarily dark, especially when red or blue is used. It is therefore either recommended to switch off the pseudocolour function of the microscope, or, if this is not possible, to later desaturate the image with image-processing software. The desaturated images, or the images originally documented as grey-scale images, need to be further processed to optimise their histograms, resulting in the use of the entire range.

If the images have been recorded with a true colour camera, the results might be very different. First, a single excitation wavelength can lead to several different emission wavelengths. This becomes especially apparent when you photograph under UV light ([27], their Fig. 4j; [32] their Fig. 4b-d). Furthermore, in many cases, distinct post-processing is necessary to remove the last remains of scattered light ([35]). In those cases, in which the specimen has been documented two times in the same position, but under different light settings or different exposure times, the two composite images can be further virtually combined, which provides comparative results to high dynamic range (HDR) recordings as used in modern cameras (e.g., [36]). When structured light is used for recording an image stack, a different set of programmes has to be used to process the stack. Most importantly, images will not be fused, but simply be projected into a single layer ([27]). Alternatively, such images can also be used for producing three-dimensional models or also simply stereo anaglyph images [27]. With the appropriate type of image processing, also non-structured light can be used for such approaches $([27,37])$.

\section{Additional comments}

Autofluorescence-based imaging has similar advantages and disadvantages as those mentioned above for reflected-light-based imaging (Table 1). Most critical is the light intensity and the deletion of scattered light to achieve the best possible contrast. Therefore, autofluorescence-based imaging under macro-photographic settings always demands a larger amount of light, while micro-photographic settings usually have a sufficient light intensity due to the built-in light sources. It is also necessary to provide a dark surrounding to reduce the scattered light, either by darkening the entire room or by an enclosed chamber around the microscope. In some cases, already a simple cardboard box might be helpful. As the exposure times are relatively long, no movement of the specimen should occur. Also, removing dust particles is crucial as these often show a larger fluorescence than the specimen and hence strongly disturb the image. Finally, especially the post-processing as described above is necessary for highly-contrasted and sharp images.

\section{Scanning electron microscopy (SEM) \\ Introduction}

Compared to light microscopy, scanning electron microscopy (SEM) has at least one essential benefit: very high magnification at excellent resolution (overview in [38]; Figs. 3, 4). This holds for both, studying surface morphology and inner organisation. Since the objects are opaque under the SEM, an inspection of internal features, however, is not possible without dissection ([39]) or freeze fraction ([40]). In addition to the high resolution, the SEM is outstanding in its tremendous depth of focus, which particularly at low magnifications is a great advantage because, compared to time-consuming 3D image processing, researchers get it almost for free. The high resolving power of SEM has been used in larval descriptions including very small and sophisticated species-specific features that can hardly be detected under the light microscope (e.g., [41-45]). SEM is also a method of choice for analyses of morphogenetic processes during larval development, for example, the development of the external morphology of larval mandibles, their shape, armature, and setation ([46]) or the morphogenetic changes from newly hatched and adult caprellids (skeleton shrimps; $[47,48]$ ). In this respect, correlative studies combining SEM with semithin histology or transmission electron microscopy (TEM) are very helpful, 

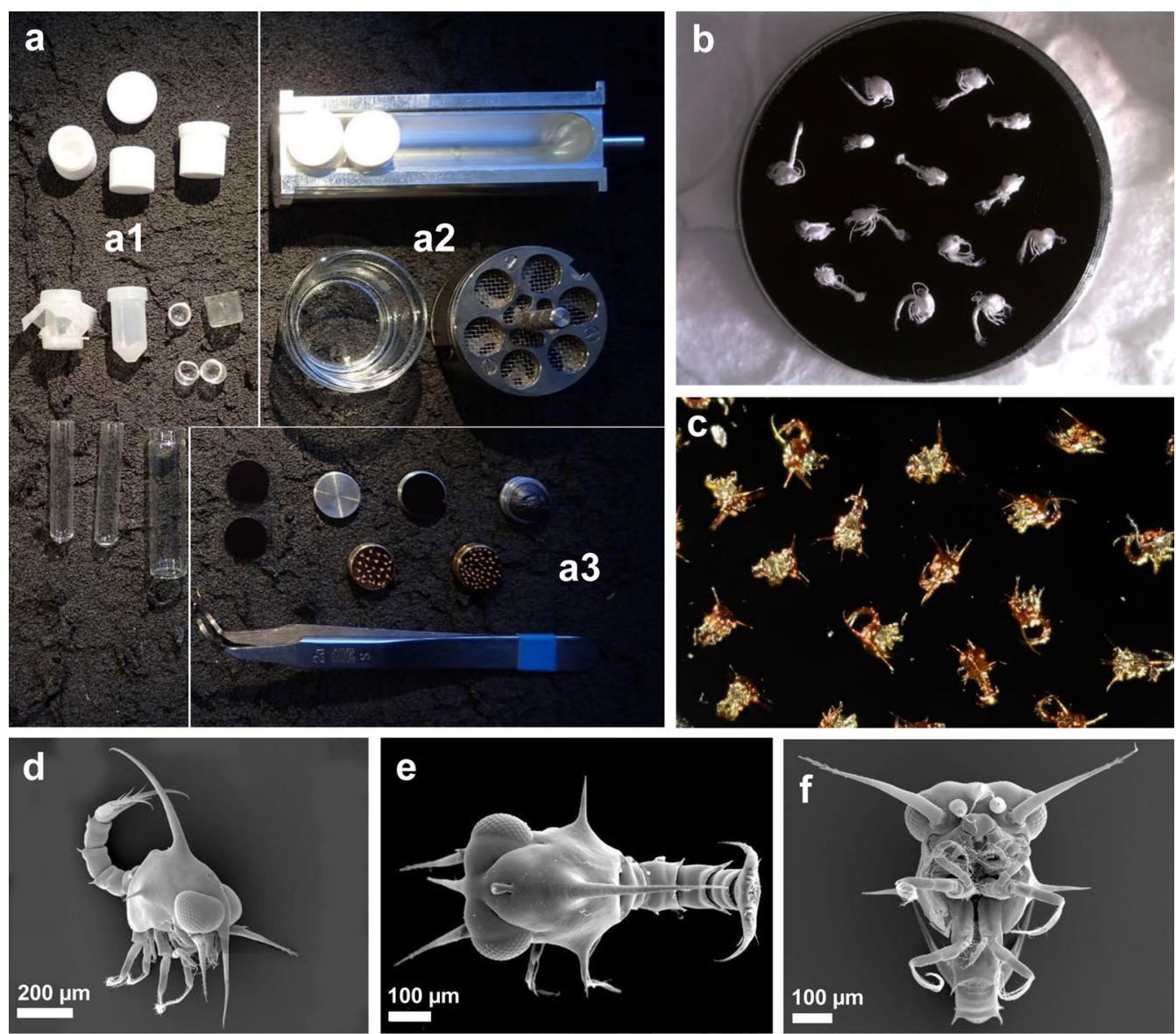

Fig. 3 Scanning electron microscopy (see section "Scanning electron microscopy (SEM)"). a Accessories for critical point and chemical drying (image R. Melzer). a1 Microporous capsules, self-made specimen holders (Eppendorf tubes with plankton mesh to ensure liquid exchange and prevent loss of larvae), small glass test tubes, and cut-off bases of test tubes to be inserted into microporous capsules. a2 Different types of holders and vials for chemical cleaning and drying. a3 Mounting of specimens: self-adhesive carbon stickers, empty SEM specimen stubs, specimen stub with carbon sticker, specimen stub with "Leit-C-plast", stub forceps, and two stubs with mounted and gold-sputtered specimens. b Higher magnification of specimen stub with cleaned, dried Zoeae of Porcellana platycheles before sputtering (image R. Meyer, R. Melzer). c "Zoea garden": Zoea 1 of Panopeus chilensis. d-f SEM pictures of Zoea 1 of Panopeus chilensis, viewed from anterolaterally (d), dorsally (e) and ventrally (f) (R. Meyer, R. Melzer, unpublished)

since thorough information about minute structures can hardly be obtained by using only one of these methods (see below). Other morphogenetic changes during larval development that can be studied with SEM are, e.g., stage-specific analyses of antennae, mouthparts, and limbs including also appendage Anlagen under the pleon (Figs. 3, 4). To use the invaluable power of the SEM to understand the three-dimensional morphology of the inner organs, the specimens to study can be dissected or deliberately broken in both, wet and dried state, to expose its inner organisation. This way, fixed, dissected and opened organs can be mounted on SEM stubs to study, e.g., gastric mills or other cuticular specialisations of the foregut and hindgut [49]. An excellent method to study soft tissues with the SEM is Tanaka-fraction [50,
51]. Here, freeze-fractured surfaces are processed with an osmium-DMSO-osmium method that allows for SEM-imaging of cracked surfaces and even intracellular structures. Scanning electron microscopy of this kind can provide important information in combined studies using various imaging techniques, whereby its resolving power is much higher than that of $\mu \mathrm{CT}$.

Many researchers will find it challenging to prepare larvae of a total length of only $500 \mu \mathrm{m}$ adequately for SEM studies. We, therefore, summarise here some handy hints and tricks on how to solve the task that we used successfully in the past decades. Optimal fixation procedures are essential (see companion paper [8]). For SEM studies, various preservation and preparation techniques must be applied to conserve the integrity of the form and surface 


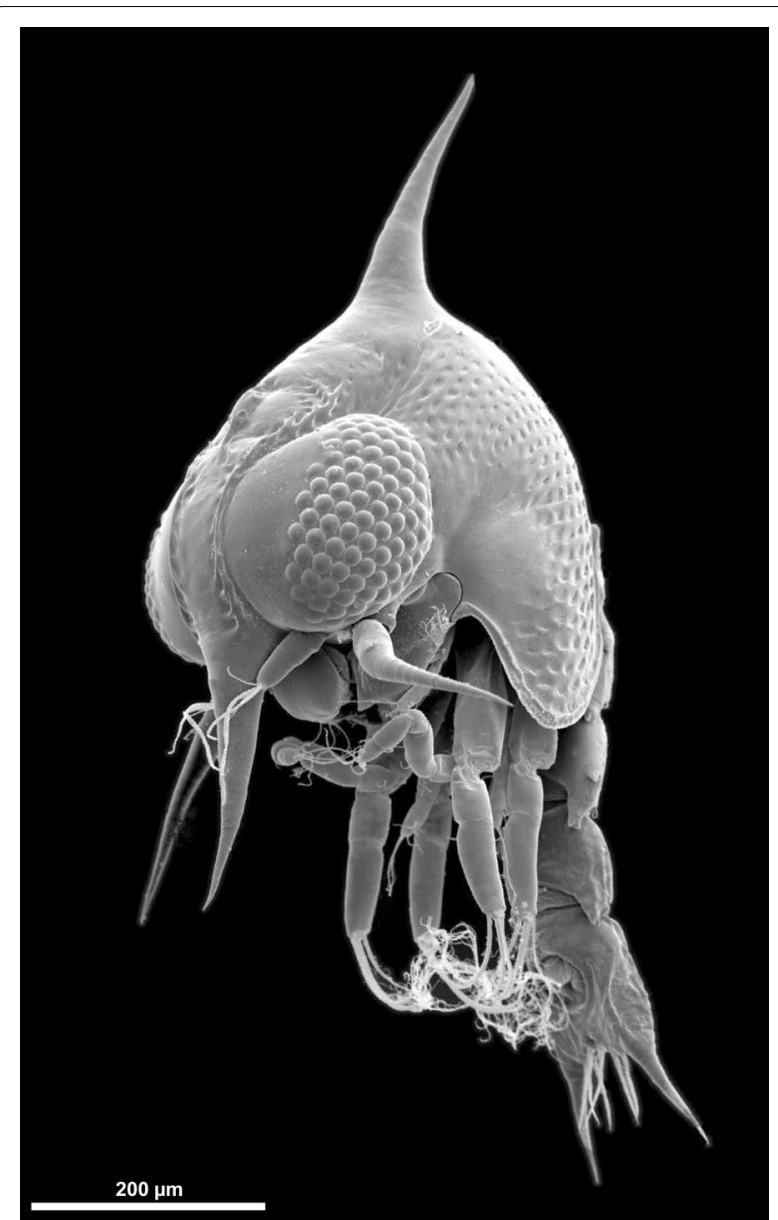

Fig. 4 Scanning electron microscopic image of Zoea 1 of Goniopsis pulchra, viewed from anterolaterally (modified from [166])

of whole or partial larvae. In most cases, good preservation requires some effort, as it will depend on the fixatives, and also on the shape and stability of the study object. For example, shrimp larvae may need different manipulation than brachyuran larvae.

\section{Description of the method}

Cleaning the larvae In our experience, a freshly hatched Zoea, or one directly after ecdysis is ideal for SEM examination. Why? Because it is clean from epibionts such as bacteria and it is undamaged. However, sometimes it will be necessary to study older larvae that have been actively feeding or have spent some time in the natural habitat. These larvae may have particles or bacteria sticking to their surface. To remove such particles there are two options the first of which is a careful mechanical cleaning (with devices such as micro brushes or eyelashes glued to toothpicks) but care needs to be taken in order not to damage the animal's delicate surface. The second option is chemical cleaning for which different methods are avail- able, for instance, using hydrogen peroxide, $\mathrm{H}_{2} \mathrm{O}_{2}$, according to Boyde [52]. Incubate the larvae in ethanol in a small dish, adding one or a few drops of $\mathrm{H}_{2} \mathrm{O}_{2}$. Small bubbles will develop on the surface of the specimen and ascend in the fluid indicating that the cleaning is on the way. After 10 or 20 min, after a refill with a little bit of $\mathrm{H}_{2} \mathrm{O}_{2}$, the larvae will be cleaner.

Drying and mounting After fixation and maybe removing surface particles, the most challenging part of producing a good SEM specimen begins, the drying and mounting. The first method suggested here is chemical drying with hexamethyldisilazane (HMDS [53-55]; mind the toxicity of this reagent), which has also been established for crustaceans [56]. The specimen is incubated in HMDS in a small dish that is covered with a glass plate, but leaving a little space to allow HMDS evaporation overnight. Sometimes, setae or other small appendages may stick together during this process. A variation of this method is drying several larvae in HMDS mounted on an SEM stub. Note however, that the orientation of the specimens on the stubs will be random. The second method is the classical critical point drying (CPD). For larvae or small arthropods in general, this approach is tricky. For CPD, the specimens need to be placed in some kind of vial or specimen holder, and after drying, they have to be retrieved again from the vials for mounting on SEM stubs. For slightly larger larvae, this problem can be solved by using microporous capsules, available in different pore sizes for every type of CPD device. They are permeable to fluids such as acetone or liquid $\mathrm{CO}_{2}$ and allow for a very good CPD. However, it is often difficult to retrieve the specimens after this process and remove them from the capsules after drying, since they might get stuck at the inner surface of the capsules. These are made of microgranules and thus have a rough instead of a smooth inner surface. An eyelash mounted on a toothpick can be used for this job. In case the samples are electrically charged, an anti-static gun (e.g., Milty Zerostat III $^{\circledR}$ ) can be used. Small Zoeae I or even parts of them need a more sophisticated setup. Usually, we use the lower part of test tubes as miniature containments for the specimens. For this, we cut off the tips of small glass test tubes (their diameter must be smaller than that of the microporous capsules) and place them into the capsules. Then, using a pipet, we put the specimens in drying acetone into the tubes, close the capsules with the lid, and insert the capsules as usual into the dryer. Because this procedure requires many and long liquid exchange steps, much practice is needed so that, with patience and a large supply of larvae, the success rate will improve. The amount of larvae available for achieving a perfect orientation is certainly an issue. A high number of specimens is important to increase the 
chances to get some of them oriented perfectly. This is relatively easy with first stage zoeae, of which hundreds can be available from a single egg-carrying female but way more difficult with megalopae when only a few are available after longer periods of larval rearing (see companion paper [8]).

Imaging It is relatively easy to obtain chemically dried larvae randomly arranged on an SEM stub. However, specific orientations of the larvae or even parts of them on the SEM stubs are required to study them in detail. Chemically or CPD-dried larvae stick well to the tips of eyelashes, glued to a toothpick, and can be transferred to the SEM stubs where they can be carefully oriented on self-adhesive carbon stickers to the desired position [41-45]. For larger specimens, a special holder that allows exposing the specimens at various angles can be used [57, 58]. Afterwards, the specimens are ready for sputter coating with gold and inspection under the SEM. For more background on preparation techniques, consult references $[59,60]$.

\section{Additional comments}

Researchers that use camera lucida or photo-based drawings [61-64] to describe larval stages, probably will not approve of larval descriptions based on SEM alone. Larval descriptions (e.g., for taxonomy purposes) must provide a standardised presentation to show the appendages always in the same orientation in a way that corresponding features are visible and can be compared between taxa. However, if the technique is well executed, corresponding views can be obtained using both drawing and SEM approaches. This double approach needs a lot of time, effort, practice, and financial resources (Table 1). Yet, it is fascinating to apply SEM on a larva, or other small crustaceans, to use the resolution of this imaging device. With this technique, differential diagnoses can be more detailed than using light or fluorescence microscopy, and minute species-specific features can be visualised that otherwise would remain undetected. In our view, the most powerful application of the SEM is the analysis of morphogenetic changes of the larval eidonomy (externally visible structures as opposed to anatomy, internal structures) during development, since changes during development can be detected at very high resolution that are not visible with other methods (see [46] for an example).

\section{Methods to study the internal anatomy Specific markers for cell nuclei Introduction}

Observing a live, moving Zoea under a fluorescence microscope with every nucleus shining blue after a vital stain is a fascinating sight. In the following, we will show that it is rather easy to obtain a larval whole mount with cell nuclei stained, dead or alive (Fig. 5). Common nuclear markers that can be used for the technique are, e.g., $\mathrm{DAPI}^{\circledR}$ (4',6-Diamidino-2-phenylindole), TO-PRO- $3^{\circledR}$, and SYTOX Green ${ }^{\circledR}$, each covering a specific section of the spectrum of light (reagents available, e.g., from Sigma, Thermo Fisher). DAPI absorbs light in the UV $(358 \mathrm{~nm})$ and emits in the blue part of the spectrum $(461 \mathrm{~nm})$, SYTOX $^{\circledR}$ Green has a maximum excitation wavelength at $488 \mathrm{~nm}$ and emits at $523 \mathrm{~nm}$, and TO-PRO- $3^{\circledR}$ is active at longer wavelengths (excitation at $642 \mathrm{~nm}$, emission at $661 \mathrm{~nm}$ ). These stains are universally used in many different fields of biosciences, but until now, they are not very common in studies of decapod development.

\section{Description of the method}

For vital stains, we recommend a protocol originally designed for Chaoborus larvae (Diptera) as described in [65], and subsequently used for Zoeae [66]. Larvae are kept in small vials in sea- or freshwater (depending on the natural habitat of the animals), and then a few drops of DAPI stain solution (ca. $1 \mathrm{mg}$ DAPI in $10 \mathrm{ml}$ distilled water) are added. After an incubation period of between $30 \mathrm{~min}$ to $1.5 \mathrm{~h}$ in darkness, the nuclei are stained and the larvae can be studied under the microscope. Where appropriate, $7 \%$ of magnesium chloride can be used to anaesthetise the larvae.

Chemical fixation of the larvae can be done either before or after staining. Unstained chemically fixed larvae should be incubated in the fluorescence marker shortly after fixation since otherwise, the staining reagent will penetrate less well as the tissues' biochemical composition becomes modified due to the action of the fixative. As fixatives, almost all formalin or ethanol-based mixtures like formalin-seawater or graded ethanol series can be used (see companion paper [8]). For imaging, the fixed specimens are mounted in an aqueous mounting medium suitable for fluorescence studies. Several companies such as Sigma or Thermo Fisher offer fluorescence media that already contain DAPI in an adequate concentration into which freshly fixed larvae can be embedded. After incubating larvae for ten to $30 \mathrm{~min}$ in these media, the nuclei of the specimen will also be stained with DAPI.

\section{Additional comments}

In cell biology and histology, fluorescent nuclear stains are commonly used as background stains for immunofluorescence labelling experiments that use specific antibodies. They help to understand the architecture of the studied organs in multichannel fluorescence analyses. Moreover, these stains can be used for nuclear and/ 

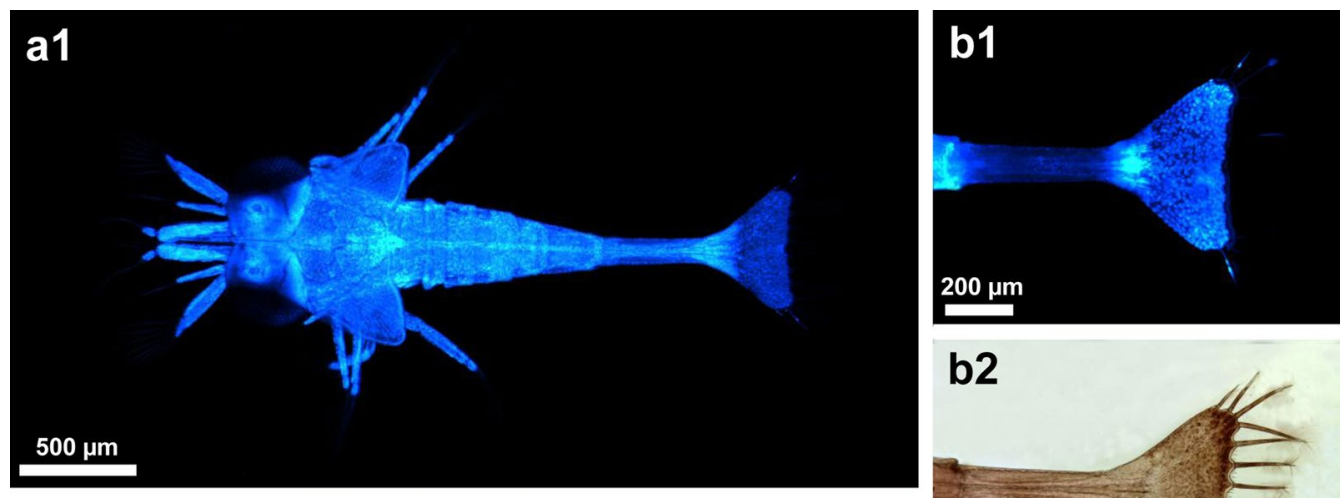

a2
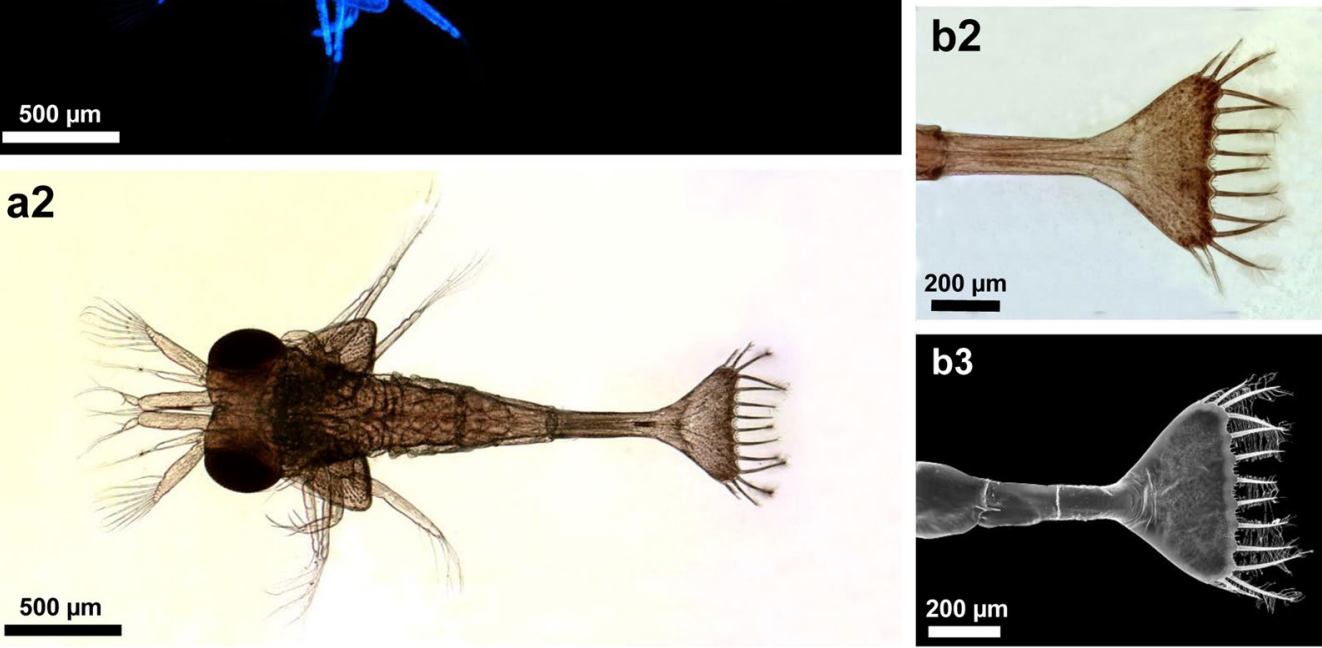

Fig. 5 Fluorescence microscopy of specific markers for cell nuclei/DNA (modified from [66]) of Zoea 1 of Palaemon elegans (see section "Specific markers for cell nuclei"). a Two-channel analysis, dorsal view. a1 DAPI-stained nuclei, a2 bright field image. b Details of pleon and telson. b1 DAPI-stained nuclei, b2 bright field image, b3 SEM. Note: all pictures, including SEM (b3), are from the same specimen

or cellular pattern analyses (e.g. [66]). We recommend employing this technique routinely as a primary tool in developmental studies of decapod larvae to study, e.g., the number and size of nuclei in certain organs or the presence or absence of clusters of densely accumulated nuclei, viz., of embryonic cell clusters. We consider it a worthwhile but scarcely used approach, e.g., to find out whether these clusters, similar to insect "imaginal discs", provide the source of morphogenetic waves of mitoses that bring about new components of the body during moults or during metamorphosis from Zoea to Megalopa. In addition to nuclear stains, there is a wealth of other fluorescent markers that allow to directly label organs that have hardly been used for larvae. For example, labelling tissue with phalloidin conjugated with a fluorescence marker was employed to study embryonic muscle development of crustaceans (review [1]) and may now be used for developmental studies on larvae.

\section{An oldie, but still a goldie—classical histology Introduction}

Classical histology is used to study biological tissues to describe an organism's microscopic anatomy and has acquired major importance since the development of the microscope in the 15th-16th century [67]. A detailed morphological and anatomical visualisation of tissues can be achieved by this technique at the micrometre scale which is essential for functional, comparative, and developmental studies of all organisms. Furthermore, using differential staining methods allows to characterise complementary histological features of the selected organs. Despite its long history and numerous competing techniques, such as micro-computed tomography or immunohistochemistry (see other sections of this paper) to analyse tissues, classical histology may still be seen as the gold standard and a frequently used approach to analyse tissues of adult crustaceans (summarised in Table 2 in [22]; see also [68-71]). Especially for small animals, this approach allows gaining insights into anatomical and morphological structures by sectioning whole specimens where other preparation or dissection is virtually impossible. To this day, serial sectioning of entire specimens is an approach regularly used to investigate morphology, anatomy, and development of decapod crustacean larvae (summarised in Table 1 of the companion paper [8]).

We summarise here the principal steps required from a living larva to a histological image. Classical histology is generally characterised by a suite of the following 
processes: (i) preparation, (ii) fixation procedure, (iii) dehydration and embedding, (iv) sectioning and staining, and (v) imaging session [72]. Steps (i) and (ii) were already described in the companion paper [8]. However, processing for transmission electron microscopy (TEM) using chemical fixation needs various additional steps, as will be detailed in the following, most of which are less important in solely light microscopic analyses. In general, microtomy (the technique of cutting an organism or single tissue into thin sections to allow for further observation under a light or electron microscope) determines the quality of tissue analysis because the thickness of the single sections and thus the resolution of the sample is defined by this step. Several modes of microtomy are classified in accordance with the section thickness, the microscopic technique, or the used microtome [72], for example: (1) histological sectioning (2-10 $\mu$; Fig. 6); (2) semi-thin sectioning $(0.25-2 \mu \mathrm{m}$; Figs. $7,8,9)$; (3) ultrathin sectioning (50-150 nm; Figs. 10, 11).

Histological sectioning based on paraffin wax (e.g., Paraplast $\mathrm{t}^{\circledR}$ ) embedded tissues presents a rapid and efficient technique to observe the overall tissue organisation. A rotary microtome is needed which is a standard piece of equipment in many histology and pathology labs. Followed by classical staining techniques, such as haematoxylin-eosin (HE) or Azan, it offers a convenient way to examine samples and differentiate larval organ systems, even with the simplest bright field light microscopes (see Table 1). Unstained histological sections can also be used in a variety of studies including in situ hybridisation or immunohistochemistry. Because classical histological sections are thicker $(2-10 \mu \mathrm{m})$ in comparison to the other techniques, this method provides an additional aspect of "depth" in the z-direction and a better insight into the coherence of tissues. Moreover, the reagents used in histological sectioning mostly are less toxic (and expensive) and therefore safer to work with. For the analysis of the internal morphology of marine crustacean larvae and juveniles, we also obtained excellent results by using histological semi-thin sectioning (section thickness of 1-1.5 $\mu \mathrm{m}$ ) of whole specimens in combination with bright field microscopy. This technique needs a higher quality microtome and profits from the use of expensive diamond knives (see Table 1). Because the specimens have to be embedded in plastic resins, it is more time consuming than the former technique. Serial section series obtained with these techniques can be used to perform 3D reconstructions.

The transmission electron microscope (TEM) is one of the mightiest (and most expensive) imaging devices in life sciences with a resolution that by far surpasses that of light microscopic methods surveyed here and, therefore, allows to analyse ultrastructure in great detail. This technique is therefore fundamental to understand the cell's ultrastructure including type, morphology, and distribution of the organelles and the cytoskeleton, and also helps to identify the cells responsible for the secretions (reviews, e.g., in [72, 73]). However, the process from the larva to the TEM image is complicated, time consuming and needs expensive chemicals and machinery (see Table 1). Hence, one should carefully consider whether the method is needed for a specific research question. For example, features such as the number of cells in an organ or analyses of the segmental organisation, or muscle fibre projections can be also unveiled with easier and faster methods than TEM. Nevertheless, whenever the development of minute structures such as sensilla, ommatidia, glands, synaptic connectivity in nervous systems and, in general, differentiation processes on the cellular level are to be studied, the TEM is the right method at hand (e.g., [74-76] for sensilla in general, and [77, 78] for crustacean larvae). Two main preparation techniques are used in TEM studies, the first is based on chemical fixation and represents the standard (see Table 1 in the companion paper [8]), whereas the second is based on cryofixation and is more sophisticated (surveys [72, 73, 79-81]). Cryotechniques circumvent some of the artefacts of chemical fixation, but are mainly useful for very smallsized specimens or objects in the $10-\mu \mathrm{m}$ range. However, if cryofixation works, more detailed analyses, a higher resolution than with the standard protocol, and therefore even analyses of macromolecules are possible [82]. As Zoea larvae are indeed very small and include numerous minute structures, this approach should maybe be used more often, especially because high-pressure freezing extends the range of suitable specimen size into the reach of Zoea larvae or parts of them. A simple method that does not need costly equipment and can be established in any laboratory is described by Steinbrecht [83]. As the authors of this paper are not experienced in cryo-TEM, we will focus on the standard technique here.

TEM analyses using chemical fixation procedures are intricately connected with their sister technique, the semithin sectioning combined with light microscopic analysis. The latter approach originally was an aid to approach the region of interest in a specimen embedded for TEM, instead of making countless ultrathin sections. However, in times of serial 3D reconstructions, it has become a powerful tool as well (e.g., [84]). Here, the principle of reciprocity is also valid, because the quality of a 3D reconstruction based on semithin sections will improve when intermittent ultrathin sections are checked in the TEM to confirm what is "really seen" with the light microscope. That way, it is possible to access where the real borders between organs are located or what the real functional specifications of a cell or a group 

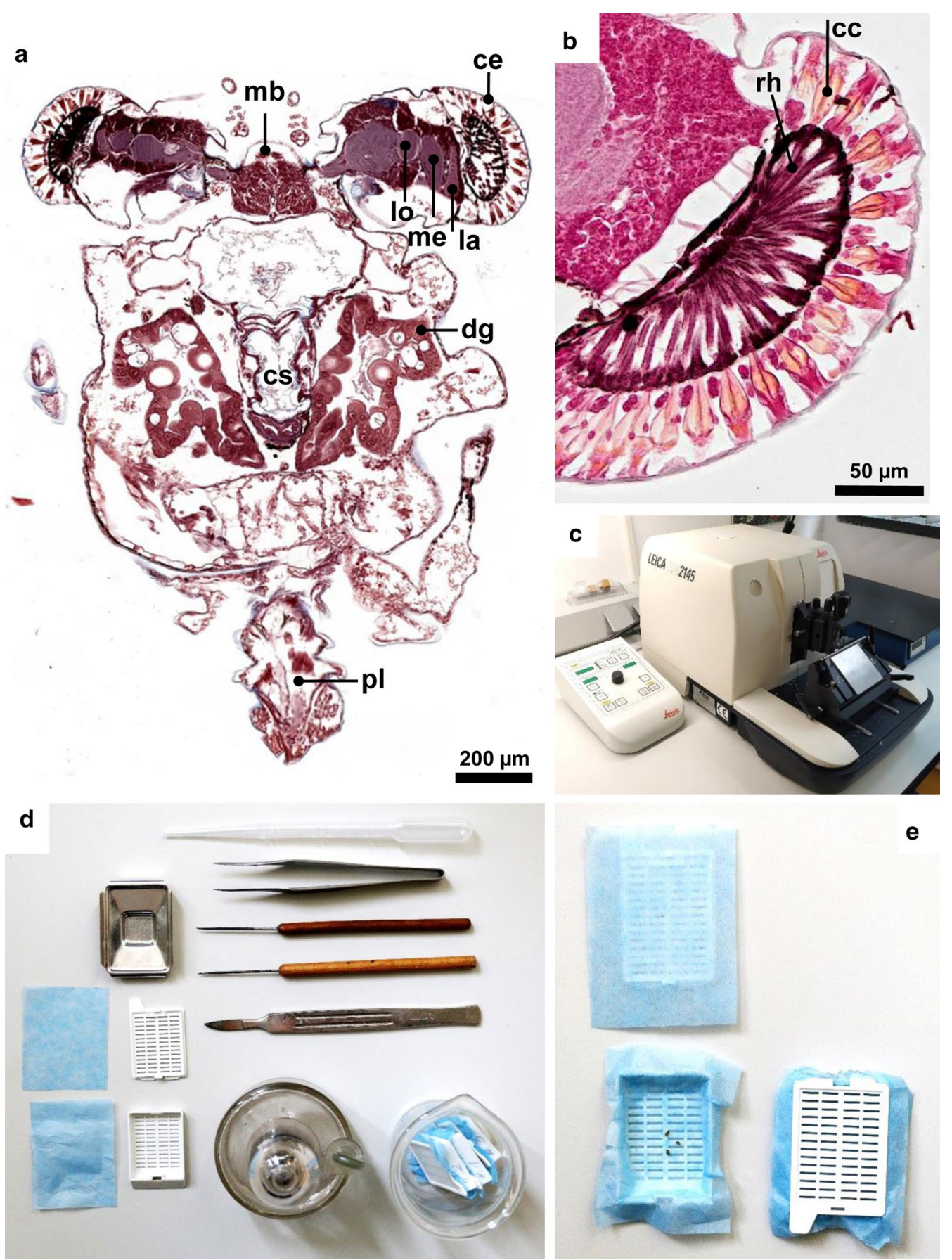

Fig. 6 Paraffin histology and Azan staining of a Carcinus maenas Megalopa (see section "Description of method: paraffin embedding, sectioning and staining (Azan)" for details). a: horizontal section of a Megalopa (Z. Šargač, unpublished). b Higher magnification of the compound eye ( $Z$. Šargač, unpublished). c Leica RM 2145 rotary microtome for paraffin sectioning (image S. Harzsch). d Various tools for paraffin embedding (image Z. Šargač). e White embedding cassettes combined with Bio-Wraps (image Z. Šargač). Cc: crystalline cone, ce: compound eyes, cs: cardiac stomach, dg: digestive gland, la: lamina, mb: median brain, me: medulla, lo: lobula, pl: pleon, rh: rhabdom

of cells might be. However, various studies are using only one line of morphological evidence (e.g., SEM), to make statements about, for example, sensillar functions. Many studies would profit from adding TEM analyses to SEM, because the crucial structure-function relationships of sensilla can be better analysed by TEM since cellular and 
dendritic ultrastructures have to be considered. There are even sensilla that are not visible at all under the SEM [77, 78].

\section{Description of the method: paraffin embedding, sectioning and staining (Azan)}

Paraffin embedding Prior to fixation, living larvae should be anaesthetised by a short exposure to low temperatures (see companion paper [8]). Before applying this method, animals must be immersed into the fixative and different appendages should be dissected (e.g., rostrum, dorsal spine, or limbs) for better penetration of the fixative into larval internal tissues (see paragraph on "fixation" in the companion paper [8]). We frequently used Bouin's fluid (i.e. $40 \%$ formaldehyde, $5 \%$ glacial acetic acid, $2 \%$ saturated aqueous picric acid) to preserve the animals. Animals completely immersed in the fixative should be stored at $4{ }^{\circ} \mathrm{C}$ for at least $24 \mathrm{~h}$. The larvae are then removed from the fixative and different methods can be applied for easier fluid exchange, depending on the size of the larvae. Larger specimens can be transferred into labelled vials and solutions can be exchanged using pipettes. However, for small larvae such as those of Carcinus maenas, we recommend using white embedding cassettes (Routine VI, Loose, Leica Biosystems; Fig. 6d, e) combined with the SurgiPath ${ }^{\circledR}$ BioWraps (Leica Biosystems). White embedding cassettes are great tools to save time and reagents because different samples can be marked separately with a pencil on the cassette itself and placed together in one beaker (Fig. 6d). To apply this technique, cut Bio-Wraps as squares to overlay the whole area of the embedding white cassette, with one centimetre extra on each border of the cassette (Fig. 6e). Place the larvae in the embedding cassette and close it with the lid merged with Bio-Wrap in a way that there is a surplus of the Bio-Wrap on each border (Fig. 6e). This technique presents an excellent substitute during solution exchange and washing on the orbital shaker since Bio-Wraps are permeable for solutions. Moreover, larvae do not stick to the wrap which thus prevents the larvae to wash through the pores of the cassette during the washing steps with agitation. Once the larvae are placed in the cassette, start the dehydration process of the samples by submerging them in ascending ethanol concentrations: $70 \%, 80 \%, 90 \%, 99 \%$ for $1-2 \mathrm{~h}$ in each step (we recommend four different concentrations, but additional steps with other concentrations could be added) at room temperature with moderate agitation (e.g., using a laboratory orbital shaker). Note that the first ethanol step (70\%) can be repeated several times until all fixative is removed. For instance, in the case of Bouin's fluid, washing in 70\% ethanol should be repeated until there is no trace of yellow colour leaching out. Especially for larger samples, washing steps can be extended to ensure successful dehydra- tion. During all the washing steps cover the beaker with, e.g., aluminium foil or Parafilm ${ }^{\circledR}$ to minimise the evaporation of the washing solutions. After the last ethanol step, submerge samples in a mixture of Tetrahydrofuran (THF) and 99\% ethanol (ratio 50:50) and leave samples at room temperature overnight with medium agitation. Tetrahydrofuran is an intermediary medium that is completely miscible with paraffin and potential residues of water in all proportions. Its use ensures the complete removal of water which results in a successful embedding in paraffin. An advantage of THF is that its vapours are much less toxic than other compounds that were widely used in the past such as dioxane (diethylene dioxide). After $24 \mathrm{~h}$, exchange 50:50 THF $+99 \%$ ethanol solution and wash larvae for another $6 \mathrm{~h}$ on a shaker. Afterwards, place the samples in clean THF and leave overnight at room temperature with medium agitation. During this step, heat paraffin granules to $60{ }^{\circ} \mathrm{C}$ using an oven or other heated chamber to ensure good fluidity of the paraffin for the next day. We use Roti ${ }^{\circledR}$-Plast with DMSO (Carl Roth Gmbh + Co. KG) granules but any other commercially available paraplast brands for histology can be used (note that melting temperatures might vary). Incubate samples in the mixture of THF and paraffin (ratio 50:50) in a $60{ }^{\circ} \mathrm{C}$ oven and let this mixture infiltrate overnight. After infiltration of the mixture, remove the larvae from the embedding cassettes and transfer them into a fresh change of melted paraffin. Considering the viscosity of paraffin, during this step, it is recommended to remove the larvae from the cassettes and put them directly in marked glassware filled with pure paraffin (for better mixing with paraffin and easier embedding later). In order to do so, pick up the cassettes with the larvae from 50:50 THF + paraffin (wearing gloves), open the cassettes, and pick the larvae with a plastic pipette (if paraffin is still liquid) or scrape them with forceps as the paraffin solidifies. Optimise this technique based on the size of the larvae and fluidity of the 50:50 THF + paraffin solution. It is important not to touch the specimens directly and minimise the surplus solution uptake during this step. Pure paraffin series can be exchanged several times until the smell of THF is gone to be certain that the samples are in clean paraffin. Because paraffin hardens quickly at room temperature, these exchanges need to be done in the heated chamber promptly. When the samples are in pure paraffin, they are ready for embedding, and we recommend not to keep for longer than a day in this medium. Prior to embedding, metal base moulds (Carl Roth + Co. KG, $52 \times 35 \times 11 \mathrm{~mm}$ outer part, $23 \times 36 \mathrm{~mm}$ inner part; Fig. 6d) need to be warmed up to $40{ }^{\circ} \mathrm{C}$. Prepare an alcohol burner and heat a dissection needle. Due to the fast hardening of paraffin, these next steps should be completed quickly and preferably by two persons (one person heating the needle and smoothening the surface 
of the paraffin and the other pouring melted paraffin and positioning samples). The first step is to pour melted paraffin into the warmed-up embedding mould so that it covers most of its area. Using a pipette, place the sample into paraffin while positioning it centrally at the bottom of the mould. We recommend arranging two to three larvae close to each other without direct contact for easier sectioning afterwards. Larvae can be positioned as desired using hot metal tools such as needles with gentle movements as the paraffin is hardening. Smoothen the surface area with a hot dissection needle to ensure that the paraffin remains liquid. Remove the lid from the embedding cassette and press the cassette into the metal mould onto the melted paraffin with the bulging part facing the liquid paraffin so that it flows through the pores of the cassette. Add more melted paraffin to the top brackets of the embedding cassette. To ensure a good attachment of the paraffin to the cassette as it solidifies, it is important to remove any residual bubbles at the top area close to the pores by using a hot needle and pushing these bubbles to the surface of the paraffin. Residual bubbles in the solid paraffin can cause the sections to rupture or can result in breaking the sample during sectioning. Leave the prepared moulds to solidify at room temperature (for faster solidification, place moulds in the fridge). When the paraffin is completely solid, cut it between the metal and plastic border (e.g., with a razor blade) and carefully separate the metal embedding mould from the embedding cassette. If a bad attachment of the samples occurs, place the whole mould again in the $60{ }^{\circ} \mathrm{C}$ chamber until the wax is liquid again. Remove the old embedding cassette carefully without disturbing samples at the bottom of the mould and repeat the steps using a new plastic embedding cassette. A hot dissection needle can be used again to rearrange the samples as desired.

Histological sectioning Prior to sectioning using a rotary microtome (e.g., a Leica Microtome RM2145), a heating plate needs to be warmed up to $40{ }^{\circ} \mathrm{C}$. Mark the cleaned microscope slides and warm them up on the heating plate. To decrease the water surface tension for an even distribution of water on the slide, place a droplet of glycine on the slide and spread it equally over the whole slide (e.g., using a paintbrush). Before sectioning, trim the solidified paraffin block with a razor blade in a trapezoid shape to reduce the surplus paraffin medium around your specimen. The blocks may be cooled for better trimming [85]. Make sure that the steel blade on the microtome is new and without any damage or physical scars to avoid section ruptures during sectioning. Position the block onto the microtome holder as desired and start trimming the block until the sample becomes more visible. Preservation in Bouin's fluid stains the samples with yellow col- our, and since the yellow Bouin's fluid penetrates deep into the tissue (especially if some appendages are cut), the yellow stain will not disappear completely. This gives the advantage during sectioning on the microtome that the samples can be easily identified in the paraffin block. Traces of the fixative will be dissolved in the later steps after sectioning, during the washing steps on the slides. However, extremely tiny or transparent samples such as those fixed in PFA (which do not leave a colour trace of the fixative) can be stained prior to the embedding in paraffin. The most widely used solutions for this are toluidine blue, cresyl violet, or a low graded iodine solution $(0.5-1 \%$ in ethanol after the final dehydration step; see previous chapter), where a drop of one of these solutions for a few seconds on the sample enhances the contrast between the sample and paraffin block. Once the sample is getting closer to the blade, switch to fine sectioning for the desired section thickness (good results can be achieved in a range of $2-10 \mu \mathrm{m}$ ). Pay special attention when the first tissue appears. Additionally, since the larvae of some species are extremely small, you can ensure the proper starting point of the sampled tissue by checking the sections under a microscope. When the first sampled tissues appear within the block, put water drops on the previously prepared heated slides and place sections onto the water surface. Since paraffin sections stick to each other, usually 3-5 sections (depending on the dimensions of the trimmed block) can be sectioned in a row, allowing for a better orientation of consecutive sections. A paintbrush, previously soaked in water, can be used to detach the sections and place them on the slides covered with water. As the heated water evaporates, the paraffin sections stretch and eventually stick to the slide. Note that transferring the sections from the blade to the slides is a tedious process and different techniques can be applied to achieve this goal es exemplified in reference [85]. Before the histological staining, the slides must be dried and can be stored at room temperature in a dark and dry place covered with aluminium foil for several months, or placed in the same way at $4{ }^{\circ} \mathrm{C}$ for even longer storage.

Staining and mounting Once the sections are dried on the slides, any desired histological stain can be used. Numerous dyes (acidic or basic) are available and can be used for staining, depending on the tissues or cells of interest. For an overview of the sample and revealing the general tissue anatomy, complex mixtures such as Periodic acid-Schiff reaction (PAS), Masson's trichrome, van Grieson, Azan, Mallory, etc. (reviewed in [67, 72, $85,86]$ ) can be used for larvae as exemplified in [23]. In principle, all stains used in analyses of adult crustacean histology such as, e.g., the popular trichromatic Masson-Goldner stain [87-90] can also be applied to sec- 
tions of larval tissues (reagents are available, e.g., from MORPHISTO ${ }^{\circledR}$ ). These methods complement each other and allow to analyse different cellular features of a given organ. A highly recommended compilation of staining techniques specific for crustaceans is available [85]. A differential analysis of an organ's histology using different stains can be complemented by immunohistochemistry (see below) to specifically localise cellular components such as elements of the cytoskeleton or transport proteins. In the following, we will elaborate on the Azan staining technique after Geidies $[72,86]$ as an example to describe steps that typically characterise a histological stain, and which we have successfully used to reveal different tissues on the paraffin sections (Fig. 6a, b). This formalin-free trichrome stain offers a great overview of the tissue, where extended washing of the samples with water does not affect the staining itself [91]. Azan staining differentiates connective and reticular tissues (blue), cell nuclei and erythrocytes (red), and muscle tissues (red-orange).

To save time and volume of exchange solutions, the slides can be placed into slide holders and stained together in one tray. The staining process starts by washing the slides with the attached sections in xylene for $10 \mathrm{~min}$ to remove the paraffin from the sections and to clear (i.e. make transparent) the tissues (any other clearing agent, such as the less toxic Roti-Histol ${ }^{\circledR}$, can be used to remove the paraffin from the sections). Transfer slides into 99\% iso-propanol and submerge them for $5 \mathrm{~min}$ to remove the xylene. Incubate slides in a descending alcohol series (we recommend a minimum of three steps: $96 \%, 80 \%, 60 \%$ ) for $5 \mathrm{~min}$ in each concentration to rehydrate the samples. For thicker sections or bigger samples, more intermediate steps could be added, such as $90 \%$ and $70 \%$ (both for $5 \mathrm{~min}$ ). Submerge the slides in distilled water for $5 \mathrm{~min}$ and afterwards incubate in a nuclear fast red solution for $30 \mathrm{~min}$ for selective staining. Dip the samples into distilled water and transfer them to $5 \%$ phosphotungstic acid to incubate for $5 \mathrm{~min}$. Phosphotungstic acid in the dye mixture provides sufficient mordanting and also ensures the right acidity ( $\mathrm{pH}: \sim 2-3)$ necessary for good results with the aniline blue-orange $\mathrm{G}$ mixture. Immerse shortly in distilled water and place slides into aniline blue-orange G solution for 10 min to stain collagen, reticular fibres and muscles. Clean the slides by shortly dipping them into distilled water, then incubate in $60 \%$ ethanol, next in $80 \%$ ethanol, and finally leave them in $96 \%$ ethanol for 5 min each step. Submerge slides in 99\% isopropanol for five min and then incubate slides in xylene for at least $5 \mathrm{~min}$ to remove potential paraffin residues. Remove the slides from the xylene, dry them out on paper tissue and by using a piece of tissue carefully remove any residual xylene drops on the slide without touching the sample. After the xylene has entirely evaporated (after a few min), mount the slides immediately with $150 \mu \mathrm{l}$ of Roti-Histokitt II (xylene-free polymeric mounting medium) per slide. Carefully cover samples with coverslips avoiding any bubbles and let the slides dry for at least $24 \mathrm{~h}$ at room temperature. Prepared dry slides can then be stored at room temperature in a dark and dry place and can last for years or even decades.

\section{Description of the method: plastic embedding, semithin sectioning, and staining with Toluidine Blue and according to Holländer and Vaaland}

Embedding in plastic resins We obtained good results (Fig. 7) with larvae fixed in FAE fixative or Bouin's solution (see section on fixation in the companion paper [8]). Because of the high toxicity of the fixatives and embedding media, all embedding steps need to be carried out under a fume hood. After washing the specimens in three changes (20 min each) of phosphate-buffered saline (PBS; $0.1 \mathrm{M}, \mathrm{pH} 7.2,1.8 \%$ sucrose), the larvae are post-fixed for $1 \mathrm{~h}$ by immersion in $2 \%$ osmium tetroxide at room temperature. The specimens are then washed in three changes (20 min each) of PBS. Afterwards, the samples are transferred to $30 \%$ acetone and dehydrated through an ascending series of acetone (in concentration steps of $10 \%)$ to $100 \%$. The dehydrated samples are transferred using micro dissecting needles in Eppendorf ${ }^{\circledR}$ plastic tubes filled with a 1:1 mixture of acetone and Araldite (Araldite ${ }^{\circledR}$ epoxy resin kit, Agar Scientific). Initially, the plastic tubes are left closed for $3 \mathrm{~h}$ and opened for $20 \mathrm{~h}$ afterwards. The completely soaked samples are then transferred into small glass dishes (embryo dishes) filled with $100 \%$ Araldite using micro dissecting needles. Also, the embedding blocks are filled with $100 \%$ Araldite. When preparing the embedding medium, the surplus of the liquid and unpolymerised $100 \%$ Araldite can be drawn into plastic syringes and stored frozen $\left(-20^{\circ} \mathrm{C}\right)$ until further use. To ensure the best quality of Araldite, the syringes need to be defrosted at least $1 \mathrm{~h}$ before use and should be defrosted not more than three times. To avoid and remove air inclusion within the specimen and in the corners of the embedding blocks, heat the glass dishes with samples and embedding blocks to $60{ }^{\circ} \mathrm{C}$ for $15 \mathrm{~min}$ and subsequently expose them to a vacuum (200 mbar) for $20 \mathrm{~min}$. In a final step, the samples are transferred into the pre-filled embedding blocks. Samples should be positioned at the tip of the embedding blocks using micro dissecting needles (Fig. 8a). The embedding blocks, including the samples, are then incubated for $48 \mathrm{~h}$ at $60^{\circ} \mathrm{C}$ for final polymerisation. The viscosity of the embedding medium decreases right before polymerisation, so we recommend checking the position of the samples after $15 \mathrm{~min}$ and eventually 


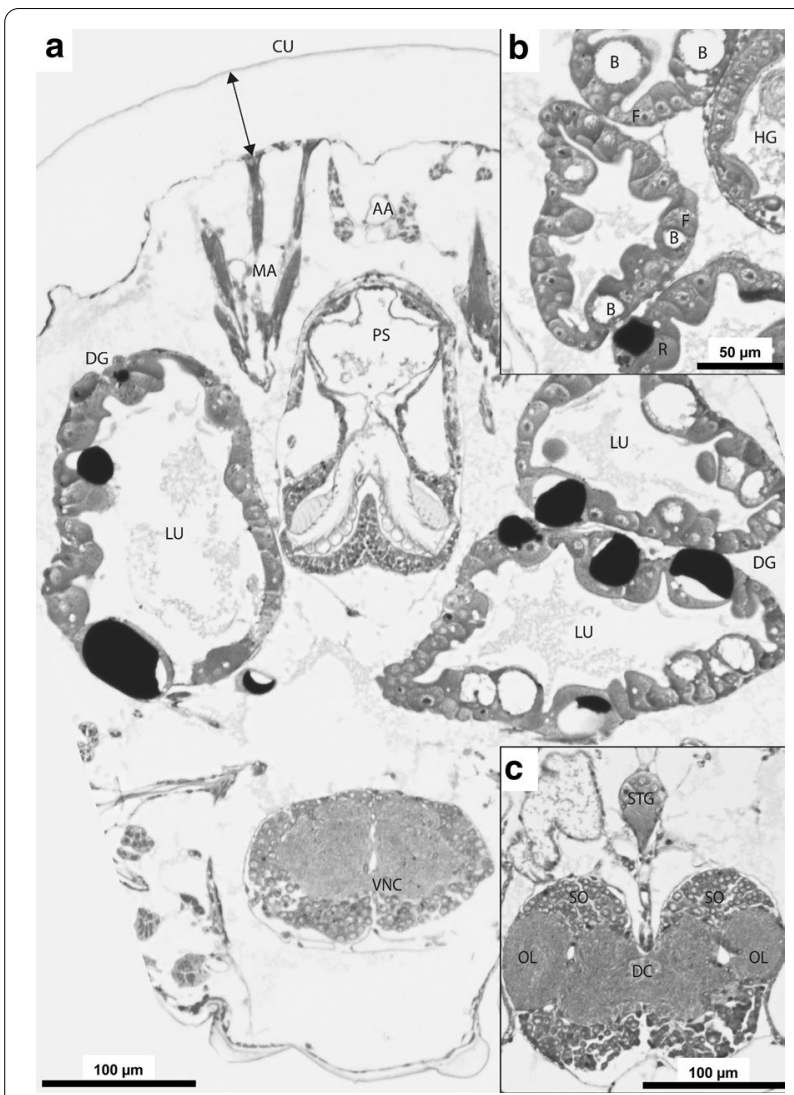

Fig. 7 Frontal semithin sections of a Megalopa of Carcinus maenas (stained according to Holländer and Vaaland 1968, [22, 92]) illustrating the resolution of semi-thin sectioning (F. Spitzner, unpublished; see section "Description of method: plastic embedding, semithin sectioning, and staining with Toluidine Blue and staining according to Holländer and Vaaland" for details). Note that the cuticle (CU) and epidermis are detached (double arrow) due to poor fixation. To avoid such artefacts, adding up to $9 \%$ non-electrolytes helps to adjust the osmolarity of the fixative and therefore avoid volume changes of tissue. a Overview section showing the median portion of the pyloric stomach (PS) laterally flanked by the lobes of the digestive gland (DG). $\mathbf{b}$ Section through lobes of the digestive gland showing epithelium with the $\mathrm{F}-$, $\mathrm{R}$-, and $\mathrm{B}$-cells surrounding the lumen ( $L U)$. c Section showing the median brain with the deutocerebrum (DC) and the dorsally positioned stomatogastric ganglion (STG). The deutocerebrum is characterised by the spherical olfactory lobes (OLs), and the neuropil of which is surrounded by neuronal somata (SO). AA: anterior aorta, B: blister-like cell, F: fibrillary cell, HG: hindgut, MA: mandibular adductor musculature, PS: pyloric stomach, R: resorptive cell, VNC: ventral nerve cord

correct the position using micro dissecting needles. The hardened embedding blocks should cool down for at least $30 \mathrm{~min}$ before further use.

Sectioning Before sectioning, thoroughly clean the microscope slides so that the sections will adhere without any folds. To that end, the adhesion microscope slides should be immersed in 96\% ethanol (or a 9:1 mixture with
$25 \%$ ammonia [84]) in cuvettes for 3 days, briefly rinsed with distilled water, and immersed in distilled water for at least 1 day until further use. Transfer the microscope slide onto a heating plate at $60^{\circ} \mathrm{C}$ until all water has evaporated (about $30 \mathrm{~min}$ ). Alternatively, the glass slides may also be used in a wet state directly for section uptake. The following descriptions are based on experiences with a rotary microtome (e.g., HM355 by Thermo Fisher Scientific). The hardened embedding block should be trimmed with a razor blade into a trapezoid, creating a truncated $45^{\circ}$ pyramid with the specimen close to front face (Fig. 8b, c). For linking sections into serial bands, the upper side of the trimmed block needs to be sealed with a 1:1 mixture of superglue and xylene (for discussion of different glues see [84]). After preparation of the embedding blocks, the sectioning knife (e.g., Histo Jumbo diamond knives by DIATOME) should be carefully cleaned with ethanol (96\%) and the collecting basin needs to be filled with distilled water. In a first sectioning step, the samples are trimmed roughly ("quick \& thick", e.g., sectioning thickness: $15 \mu \mathrm{m}$; sectioning speed: 10 units in our device-specific setting), and trimming needs to be stopped before the embedded sample is reached by the knife. For semi-thin sectioning, the process needs to be slowed down (sectioning speed: 5 units), and sectioning thickness should be reduced (sectioning thickness: $1-1.5 \mu \mathrm{m}$ ). The single sections should stick together in a band due to the one-sided sealing with superglue [84]. The length of the section bands depends on the operator's experience and sample size, but should not be longer than 15 attached sections as the sections unfold (and thus expand a little) when drying on the heating plate. The section band needs to be lifted carefully from the knife and left floating within the water bath. Afterwards, the microscope slide needs to get immersed carefully into the water bath. The microscope slides should be moved carefully underneath the section bands and carefully lifted out of the water. When immersing the microscope slides underneath the section chain, it is useful to reduce the surface tension of the water bath by placing a pipette crosswise in front of the microscope slide (Fig. 6d).

Staining and mounting The staining after Holländer and Vaaland [92] (1\% phenylenediamine in methanolisopropanol) strongly stains nervous tissues. Moreover, it has been shown that phenylenediamine also stains nucleoli and cytoplasm of other cell types as well, and therefore allows visualisation of several types of tissues within a crustacean larva (Fig. 7). In this method, it is likely that the formation of highly stained oxidation products by phenylenediamine in combination with the reduction of tissue-bound osmium plays an important role [92]. As osmium tetroxide (from the post-fixation 

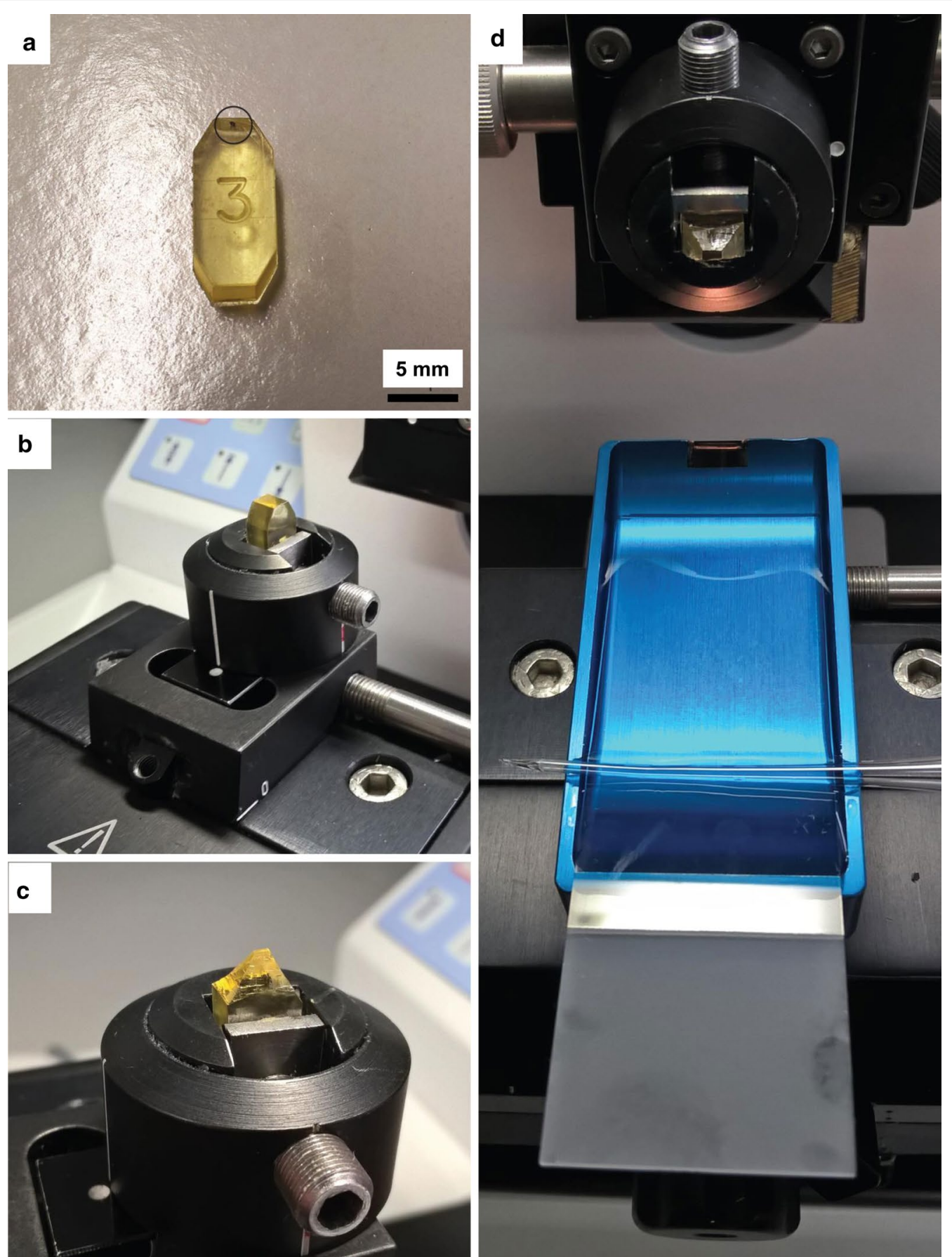

\section{C}

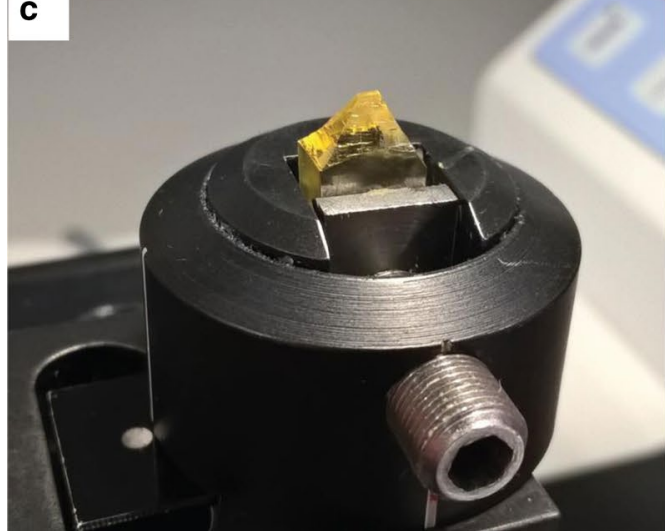

Fig. 8 Semithin sectioning using the rotary microtome (images F. Spitzner; see section "Description of method: plastic embedding, semithin sectioning, and staining with Toluidine Blue and staining according to Holländer and Vaaland" for details). a Sample of a Zoea I of Carcinus maenas embedded in Araldite ${ }^{\circledR}$ (black circle). b Clamped embedding block before trimming. c Clamped embedding block after trimming into trapezoid shape to reduce cutting surface and duration of sectioning. $\mathbf{d}$ Lifting the section chain out of the water bath. It is useful to reduce the surface tension of the water bath by placing a pipette crosswise in front of the microscope slide when carefully immersing the microscope slide into the water bath (see section "Description of method: plastic embedding, semithin sectioning, and staining with Toluidine Blue and staining according to Holländer and Vaaland" for details) 

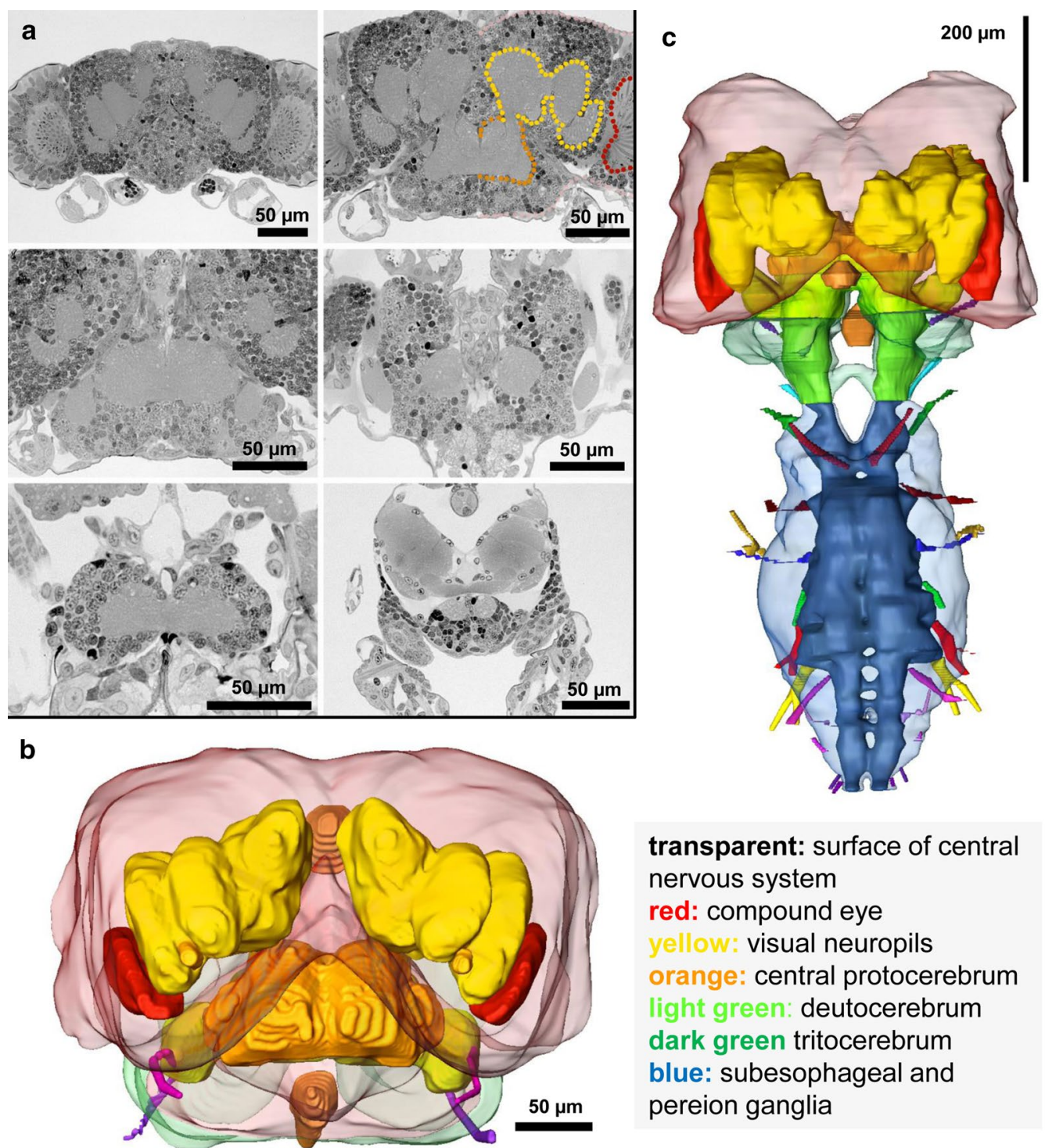

\author{
transparent: surface of central \\ nervous system \\ red: compound eye \\ yellow: visual neuropils \\ orange: central protocerebrum \\ light green: deutocerebrum \\ dark green tritocerebrum \\ blue: subesophageal and \\ pereion ganglia
}

Fig. 9 3D-reconstruction from serial semithin sections; Zoea 1 of Hippolyte inermis (modified from [167]). a Six sections as examples from a series of transversal semithin section series. The dotted lines indicate the neuropil borders used for the 3D-reconstruction in $\mathbf{b}$, and $\mathbf{c}$ ). $\mathbf{b}$ 3D-reconstruction of median protocerebrum, visual neuropils and brain surface (transparent) in frontal view. c Central nervous system with neuromeres, surface (transparent), and segmental nerves. Surface renderings were made in Amira 5.2.0. Colours: transparent red—surface of protocerebrum, transparent green — surface of deutocerebrum and tritocerebrum, transparent blue — surface of suboesophageal and pereion ganglia, red — retina of compound eye, yellow—visual neuropils, orange—-median protocerebral neuropils, light green—neuropils of deutocerebrum, dark greenneuropils of tritocerebrum, blue-neuropils of suboesophageal and pereion ganglia

process) only stains unsaturated lipids, while phenyldiamine does not stain lipids, it could be shown that the combination of both reagents results in enhanced staining of unsaturated lipids and some staining of saturated lipids [93]. The following steps should be carried out under the fume hood due to the high toxicity of the reagents. The microscope slides with the semi-thin sections should be incubated in the staining solution for ca. $14 \mathrm{~h}$.
Afterwards, the microscope slides need to be taken out of the staining solution and washed three times for $20 \mathrm{~min}$ each with iso-propanol. The microscope slides need to be rinsed for another $3 \times 5 \mathrm{~min}$ with distilled water and dried afterwards on a hot plate. As an alternative to the stain presented above, good results were also achieved using Toluidine blue ( $1 \%$ Toluidine blue, $0.25 \%$ Pyronin G in a solution of $1 \%$ sodium tetraborate, [94]). Note however, 

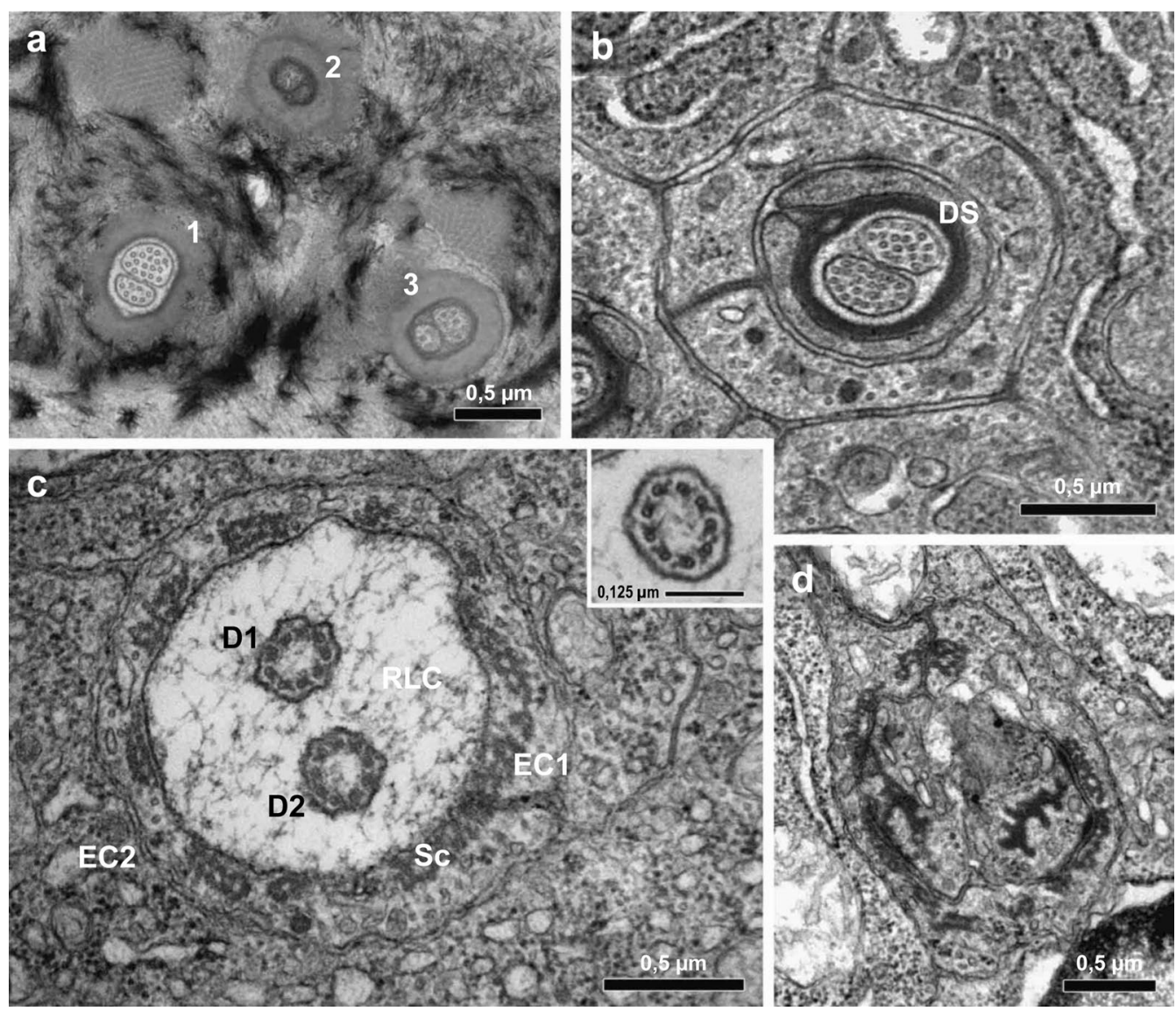

Fig. 10 Transmission electron microscopic images of serial ultrathin transversal sections (see section "Description of method: ultrathin sectioning and transmission electron microscopy" for details) through a triplet of sensilla (1-3), each comprising two sensory cells and their dendrites (D1, D2), on the molar process of the mandible of larval Palaemon elegans revealing cellular ultrastructures indicative of the architecture and function of the sensilla (modified from [77]), a: distal dendrites near sockets of setae. b-d Transversal sections at different levels from distal (b) to proximal (d). c Dendrite with $9 \times 2$ \& 0 pattern of microtubules and A-tubulus with arms. Abbreviations: DS: dendrite sheath, EC 1, EC2: enveloping cells 1, 2, RLC: receptor lymph cavity, Sc: scolopale

that in all available stains for plastic embedded tissues are suitable for larvae also (reviewed in $[67,72,85]$ ) and we describe only those stains with which we have hands-on experience. Both staining solutions described here should be prepared at least $24 \mathrm{~h}$ before use to ensure that all crystals are dissolved. Toluidine blue, also known as tolonium chloride, is a dye with a high affinity for acidic tissue components and organelles rich in DNA and RNA such as, e.g., the cell nucleus [95]. This stain is a fast technique since the sections need to be covered with the staining solution only for a few seconds (ca. $30 \mathrm{~s}$ at room temperature). For both staining techniques, it is advisable to check the results in between as these stainings are nearly irreversible and overstaining makes it difficult to analyse sections due to dark sepia or blue tones. After staining, the microscope slides should be rinsed several times with distilled water until no more dye bleeds into the washing medium, and finally dried on a heating plate $\left(60{ }^{\circ} \mathrm{C}\right)$ for approximately $30 \mathrm{~min}$. The final step before microscopy is mounting the coverslips on the microscope slides to protect the sections. We recommend using Roti $^{\circledR}$-Histokitt II to cover-slip the specimens. Three small droplets should be put on each microscope slide, covered slowly with coverslips (avoiding bubbles), and left to dry for $24 \mathrm{~h}$ at room temperature.

\section{Description of the method: ultrathin sectioning and transmission electron microscopy}

Plastic embedding The dissection and fixation of the specimens for TEM while cooling the samples on ice are described in the companion paper [8] and briefly summarised in the introduction to Sect. 2 of this contribution. The next step, in traditional protocols, is dehydrating the specimens in a graded series of $30 \%, 50 \%, 70 \%, 90 \%$, and $96 \%$ ethanol followed by $100 \%$ isopropanol, $100 \%$ 


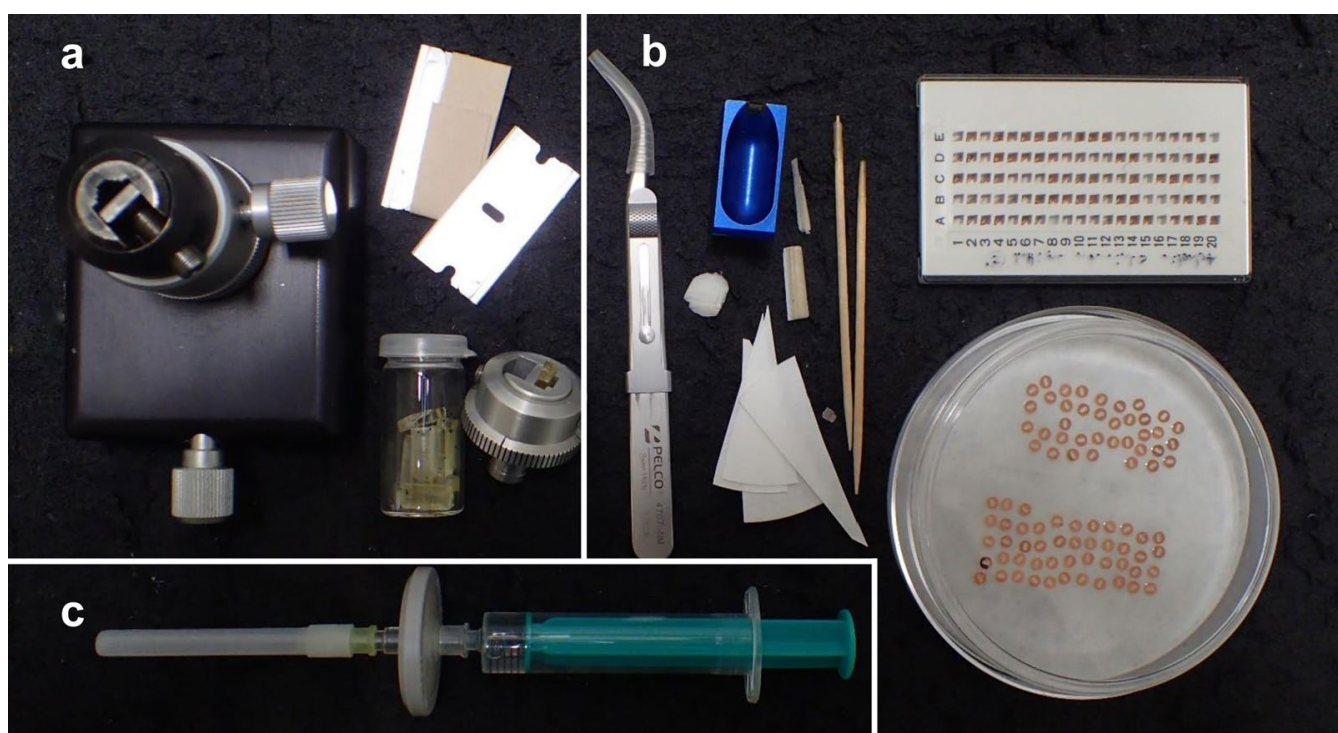

Fig. 11 Equipment for ultrathin sectioning (image R. Melzer; see section "Description of method: ultrathin sectioning and transmission electron microscopy" for details). a Accessories for trimming: specimen holder socket, razor blades, vial with plastic-embedded specimens, specimen holder with trimmed specimen. $\mathbf{b}$ Tools for sectioning and fishing sections from the knife's water bath. Forceps with bent tips for grid handling, diamond knife for ultrathin sections, filter paper tips for drying grids, tooth sticks with eyelashes glued onto tips, sharpened pieces of elder marrow for cleaning of diamond knife, grid holder, Petri dish with formvar-coated copper slit grids for serial ultrathin sections. c Syringe with microfilter for the various steps during sectioning that require absolutely clean double-distilled water

propylene oxide, and then a mix of propylene oxide and resin $[73,96]$. In many labs, however, this procedure was replaced by a graded acetone series, because acetone is a good solvent for epoxy resin. In addition, $100 \%$ acetone needed for the last dehydration steps can be kept free of water quite easily on granular calcium chloride. A feasible dehydration protocol would thus be $30 \%, 50 \%, 70 \%, 90 \%$, $100 \%$ acetone for $10 \mathrm{~min}$ each, and then 3 times $20 \mathrm{~min}$ dried pure acetone. At the $70 \%$ acetone step, the specimen vial should be taken from the ice bath and exposed to room temperature, avoiding surrounding water, because otherwise there is a risk of accidental rehydration of the specimens. Afterwards, the specimens are incubated in a 1:1 mix of acetone and resin for $30 \mathrm{~min}$ to $2 \mathrm{~h}$ maximum, and then in the pure resin. This step can last overnight, but after a few hours, the lids of the vials should be removed to allow the acetone to evaporate. After that, the specimens are placed in fresh unused resin into embedding forms and oriented according to the desired sectioning angles. Some typical accessories for carrying out these and the following steps are shown in Fig. 11.

Osmium tetroxide, and cacodylate buffer, as well as aldehydes and the resin components, are very poisonous and have to be handled according to strict safety rules. Hence, the use of gloves and eye cover when preparing the solutions is essential. Always leave the solutions under a fume hood, and if possible, use a special "preparation hood" where also the dissecting microscope is placed.

Preparing the epoxy resin needs some preliminary considerations, since resins of different hardness can be obtained depending on the proportion of the resin's components. In most epoxy resins (Epon, Araldite ${ }^{\circledR}$, Glycidether 100, etc.), there is a soft component and a harder one. Hence, it is possible to prepare resins whose hardness matches the needs of the specimens to study. After the two resin components are mixed together, the accelerator/activator is stirred in according to the manufacturer's instructions. For soft specimens, medium to soft resin is suggested, for hard ones (e.g., those containing hard arthropod cuticle), a harder resin is recommended.

Once the specimens are placed in resin into the embedding form, they are heated in an incubator or heating cabinet at $40{ }^{\circ} \mathrm{C}$. Initially, the resin becomes more fluid due to the rise in temperature which can improve its infiltration. After about an hour, before the resin becomes waxy, there is still opportunity to improve the specimens' orientation within the embedding form, if necessary. After that, the specimens are put back into the oven and the temperature is increased to $60^{\circ} \mathrm{C}$ overnight so that the resin polymerises completely. After removing the specimens from the incubator, they should be left 
in the embedding form until they have cooled down to room temperature. Fresh from the incubator, they are still quite soft and flexible but at room temperature, they will become hard. An alternative to this basic technique is vacuum embedding [97], a technique which is often successful in specimens that prove difficult to be embedded using the standard protocol.

Sectioning Trimming, sectioning, and staining are in principle quite similar to semithin sectioning as described above (Fig. 8). Nevertheless, some specific tricks and techniques to obtain good ultrathin sections will be described in the following (see also Fig. 11). An ultramicrotome (e.g., Leica Microsystems) instead of a simple rotation microtome is needed and a diamond knife for ultrathin sections (e.g., DIATOME ${ }^{\circledR}$ ) is strongly suggested instead of glass knives. With a diamond ultrathin knife, good sections can be made as a standard.

In the following, we refer here to some aspects which in our experience are crucial for a successful ultrathin sectioning and subsequent TEM analysis. As suggested in the previous section about semithin sections, the trimming (with a razor blade) should create a truncated $45^{\circ}$ pyramid with the specimen close to the front face having the shape of a trapezoid. In small arthropods, such as larvae, it is usually better not to trim up to the specimen but leave a little resin edge around. It is important that the two parallel edges of the trapezoid, which will become the upper and the lower edge of the sections, are perfectly parallel to get straight ribbons of ultrathin sections instead of spiral-shaped ribbons. It is important to practice adjusting the block in reference to the diamond knife. It needs much experience to operate the micrometre screws for adjusting the block and the knife in combination with the upper and lower illumination devices of the microtome. This allows to precisely adjust all three angles using the "shadow line" between block and knife to allow for effective sectioning. With a well-trimmed block and a good knife, sectioning of very good ultrathin sections is not as difficult as beginners often think. For high resolution, the sections should be really thin, of grey or silver interference colour $(40-70 \mathrm{~nm})$. For relatively low resolution, sections of golden interference $(70-90 \mathrm{~nm})$ are sufficient. In ultrathin sectioning, silver sections are, therefore, more of a gold standard than gold sections. As in semithin sectioning, another essential issue is "fishing" the sections from the water bath attached to the knife and transferring them to the grids in perfect orientation. This can be done from above or below-resulting in mirrorsymmetrical pictures in the TEM-with the help of formvar coated copper "slit" grids and a clean eyelash glued to a tooth stick, a basic tool of the ultramicrotomist. It can be used to split ribbons of sections into handy pieces and to orientate the sections along the grid edge. Another important trick is to stretch the sections with a little bit of chloroform vapour emitted by a piece of moist filter paper held close to the grid but not touching it. One more parameter is essential for the entire procedure, the sectioning angle, usually indicated for each diamond knife in the manufacturer's instructions. However, on some occasions, this angle should be slightly adjusted. If the section thickness is not homogenous within a ribbon of sections, increase the angle; if there are fissures or cracks within the sections, decrease the angle.

\section{Additional comments}

Despite its long history and numerous competing technical approaches, classical histology is still a commonly used method to investigate organisms' cells and tissues. While histological (paraffin) sectioning is a fast and fairly simple approach to analyse organs of interest, higher-resolution methods, such as semi-thin and eventually ultrathin sectioning provide additional insights into cells and tissues of organisms only a few millimetres in size such as crustacean larvae (Figs. 6, 7, 10). Another advantage of classical histology is the long-term stability of the finished samples. When stored correctly (dark, cool, dry), the sections (as well as the embedded blocks) can last for decades. Nevertheless, classical histology is technically challenging, and a highly invasive method as it leads to alteration and destruction of the specimen. For instance, paraffin embedding is not suitable for extremely hard samples, such as strongly calcifying organisms, where due to softer paraffin, samples usually break during the sectioning process. Furthermore, this procedure is prone to artefacts such as section loss, distortion, and staining artefacts. In addition, classical histology is time and labour consuming, as well as costly because expensive microtomes and microscopes are needed (see Table 1). Compared to light microscopy-based histology, a TEM analysis requires more work and time, but the higher resolution power from the TEM pays off when results about minute structures and cellular ultrastructures are needed.

Overall, to date (and not only limited to samples of invertebrates), the combination of histological sectioning, staining, and microscopic assessment of 2D sections, is still the gold standard for structural and compositional analysis of biological tissues [98]. Serial sections are excellent starting material for $3 \mathrm{D}$ reconstructions of selected organ systems such as the nervous system (Fig. 9). In a broader perspective, it has been shown that the combination of multiple analytical methods with classical histology might be the most effective approach to gain a holistic insight into the anatomy of invertebrate 
organisms (e.g., [22, 23, 98, 99]) because single methods sometimes cannot fulfill all requirements. Nevertheless, the selection and combination of methods depends on the objective and certain species characteristics and therefore, should be chosen with care.

\section{X-ray microscopy $(\mu \mathrm{CT})$ Introduction}

The application of X-ray microscopy $(\mu \mathrm{CT})$ to soft tissues has now become a standard in imaging techniques (Figs. 12, 13). In the field of anatomy, $\mu \mathrm{CT}$ has been demonstrated to be a useful analytical tool applied in a rapidly growing variety of metazoan taxa, such as cnidarians [100], plathelminths [101], nematomorphs [99], nematodes [102], polychaetes [103, 104], molluscs [98, 105], echinoderms [106, 107] as well as arthropods [108-113]. Modern, lab-based $\mu \mathrm{CT}$-scanners deliver high resolution allowing the investigation of tiny specimens with body sizes of free-living crustacean larvae ranging from 75-195 $\mu \mathrm{m}$ in Tantulocarida [114], the smallest arthropods in the world, up to $5 \mathrm{~cm}$ in length, e.g., in Stomatopoda [115]. Since it is a non-destructive method allowing to investigate intact specimens as a whole (reviews [109, 116]) as well as in the anatomical context (Figs.12, 13), $\mathrm{X}$-ray microscopy opens up numerous opportunities for correlative microscopic workflows such as the combination with serial block-face imaging [117], scanning- or transmission electron microscopy [118], including histological sectioning for light microscopy $[98,118]$, or the combination with magnetic resonance tomography [119] of one and the same specimen.

\section{Description of the method}

In general, imaging of biological samples based on $\mu \mathrm{CT}$ results from different absorption coefficients of tissues and of how X-ray radiation is absorbed by these samples. Thus, X-ray absorption is dependent on the atomic number based on the tissues' biochemical composition [120]. The best results in terms of resolution, reduced scanning time, and contrast can be achieved by scanning samples that were previously fixed, contrast-enhanced and critical point dried. For a better resolution of integumentary structures, dried specimens can be additionally coated analogous to the sample preparation (sputter coating) for scanning electron microscopy [121].

Here, we briefly summarise methodological steps of the sample preparation for a workflow enabling a consecutive immunohistochemical and/or histological investigation (for further details of other possible tissue preparations see our extensive manual on $\mu \mathrm{CT}$ in decapod larvae [122]). First of all, the larvae need to be properly fixed. Note that the choice of fixative has an effect on tissue preservation as well as the choice of agents for contrast-enhancement. Bouin's solution or $4 \%$ paraformaldehyde (PFA) in phosphate-buffered saline (PBS) or seawater have turned out to be useful fixatives that allow immunohistochemical labelling after $\mu \mathrm{CT}$-scanning (see companion paper [8]). After a proper fixation for at least two hours at room temperature, the tissues need to be post-fixed in Dent's fixative (20\% DMSO in methanol) and further dehydrated in two graded steps up to $100 \%$ methanol. At this stage, larvae can be stored at $-20{ }^{\circ} \mathrm{C}$. Note that fixation with methanol disrupts or denatures binding sites of some epitopes in tissues to some extent (e.g., of F-actin resulting in a limited detectability by, e.g., phalloidin conjugated with fluorescent dyes). Ethanol or isopropanol represent an alternative to methanol. However, depending on the scientific question, any of the subsequently used staining agents need to be carefully checked in advance for their compatibility with methanol-fixed tissues. After dehydration and before scanning, the tissues should be incubated in $2 \%$ iodine in methanol for contrast-enhancement for at least $24 \mathrm{~h}$ at room temperature. After two to three washing steps using pure methanol, the larvae can be directly scanned ideally in sealable plastic chambers filled with fresh methanol (for further details on mounting of samples, see [122]). After successful scanning, the sample needs to be rehydrated in a decreasing series of methanol in TRIS-buffer and finally be washed in several changes of PBS-TX $(0.3 \%$ Triton $\mathrm{X}-100$ in $0.1 \mathrm{M} \mathrm{PBS}$ ) to remove any reminiscent iodine from the tissue. After washing, the tissues are ready for any further processing for histological sectioning followed by immunohistochemical or histochemical treatments.

\section{Additional comments}

The main advantage of X-ray microscopy is that it is a virtually non-destructive method allowing to investigate intact specimens as a whole. Furthermore, this technique provides a high resolution even to the cellular level in well-fixed and contrasted specimens. Finally, $\mathrm{X}$-ray microscopy opens up numerous opportunities for correlative microscopic workflows of one and the same specimen. For example, we established workflows allowing for combining $\mu \mathrm{CT}$ with consecutive immunohistochemical (see next chapter) or histochemical labelling of the same specimen, and also combining $\mu \mathrm{CT}$ of Cobalt-filled axonal projections with subsequent sectioning [123]. This way, general information on the spatial relationships of the whole internal anatomy can be assembled before using other destructive techniques such as histological sectioning. In other words, the collection of $\mu \mathrm{CT}$-based datasets can be considered as an anatomical backup and is therefore also used for taxonomy in zoological museums or collections for digital 
preservation of type material being referred to as cybertypes $[110,124,125]$. For the study of type material, the undesirable brown staining of specimens due to the use of agents for contrast enhancement such as $2 \%$ iodine in ethanol is fortunately reversible. These agents can be completely removed after enduring (several days) of washing in several changes of fresh volumes of pure ethanol (Fig. 14) before transferring the samples back to $70 \%$ ethanol as often used in collections. Since $\mu \mathrm{CT}$ imaging is a less-destructive method, it is one of the few techniques enabling scientists to have a look into the inside of, for instance, rare fossil specimens such as amber inclusions of, e.g., insect larvae (e.g., $[126,127])$ or Cambrian arthropods [128]. A re-investigation by applying $\mu \mathrm{CT}$ of already described fossil crustacean larvae [129-131] would thus be feasible. Here, nature already embedded the samples millions of years ago.

Another advantage is that $\mu \mathrm{CT}$ produces isometric data which are favourably useful for morphometric approaches in contrast to anisometric imaging such as, e.g., microscopy of serial sections or confocal laser scanning microscopy where z-axially refractive mismatches can occur $[111,132]$. Some possibilities to visualise these data sets are exemplified in Fig. 12. One of the most commonly used analytical processing is to generate $3 \mathrm{D}$ reconstructions of selected organs or tissues (Figs. 12, 13); and see Table 2). In principle, 3D reconstructions can also be generated from microscopic images based on section series (Fig. 9). However, image stacks based on $\mu \mathrm{CT}$ provide isometric datasets meaning that the pixel-size equals in all three dimensions (depending on the scanning-settings and magnification). Furthermore, artefacts only occur due to chemical fixation, drying of samples, or scanning. In contrast, image series generated from sections often suffer from more artefacts and need to be aligned before they can be used for 3D reconstruction. In addition, these datasets are anisometric which means that the pixel-size especially of the z-axis is ultimately dependent on the physical section thickness, or if generated using a confocal laser scanning microscope, the pixel sizes have to be manually corrected due to possible refractive mismatches caused by the immersion media.

Because $\mu \mathrm{CT}$-imaging is, to put it simply, based on differences in tissue densities, it is less specific than, e.g., techniques using histological staining or antisera raised against a variety of antigens. The major disadvantage is of course that the acquisition, as well as the service and maintenance of a lab-based $\mu \mathrm{CT}$-device are quite expensive (see Table 1). What is more, sample preparation and $\mu \mathrm{CT}$, like most other techniques, also have specific disadvantages. For delivering good contrast, especially soft tissues need to be fixed and incubated in contrastenhancing agents in advance which often cause tissue modifications such as shrinking (see [111, 122]). However, by using methanol as outlined above, we obtained excellent results for later immunohistochemical labelling against anti-synapsin (SYNORF1) in combination with histochemical detection of the cell proliferation marker EdU previously in vivo injected (Krieger, unpublished). The possibility of combining these techniques makes the protocol especially interesting for studying developmental stages such as larvae. If specimens are not intended to be used for additional staining experiments or immunohistochemistry, $\mu \mathrm{CT}$ of critical point dried samples delivers better contrasts as resulting from wet-scanning in, e.g., high-grade alcohols $[22,122]$. Furthermore, by using dried samples, a specimen container becomes redundant so that the working distance can be decreased resulting in higher resolutions and shorter exposure and thus scanning times. However, dried material can be still later used for scanning electron microscopy (SEM).

\section{Methods for cellular analyses Immunohistochemistry Introduction}

Classical histological techniques provide insights into the general organisation of tissues and organs. By using primary antibodies that bind to specific cellular antigens, immunohistochemistry allows to selectively label a broad range of cellular components ranging from signalling molecules across elements of the cytoskeleton, across macromolecules such as channels or transport proteins to organelles such as mitochondria [133, 134]. One major strength of this technique is that in double or triple labelling experiments, primary antisera from different host species (e.g., rat, mouse, and rabbit) can be combined to label multiple antigens (Fig. 15).

Choosing suitable primary antibodies, according to the question, is an essential first step when designing an experiment. In general, the antibody toolbox of developmental biologists working with non-genetic model organisms is much smaller than that available, e.g., for the study of $D$. melanogaster, C. elegans or vertebrate model species. Considering aspects of both cross reactivity and specificity is an important issue when using antisera that were not raised against crustacean antigens so that identifying antisera that specifically bind in crustacean tissues may be a challenge. Therefore, suitable control experiments to test the specificity of the antisera is an essential measure to evaluate the results and often is a prerequisite for publishing the results $[133,134]$. For those new to the trade, we recommend to start using antisera against antigens which are highly conserved in an evolutionary sense such as elements of the cytoskeleton. The Developmental Hybridoma Bank Iowa (DSHB) is a valuable source for such reagents. For any new antiserum you 
work with, you should conduct a pilot experiment to find a suitable dilution (1:10, 1:100, 1:1,000, 1:10,000 etc.). If your signal-background ratio is unsatisfactory, decrease the concentration of the primary antisera to reduce unspecific background. Considering decapod crustacean larvae, one focus of research are ontogenetic aspects of osmoregulation (references in Table 1 of the companion paper [8]). Immunolocalisation of ion pumps such as the sodium-potassium ATPase in tissues specialised for ion transport is an essential method in these studies. Immunohistochemistry against neurotransmitters, neuromodulators and neurohormones is another research focus in decapod larvae involving immunohistochemistry. This technique has been widely used to analyse the maturation of neurons in the emerging brain in several crustacean species especially with respect to the maturation of the X-organ/sinus gland complex (references in Table 1 of the companion paper [8]). More than in larvae, immunohistochemistry has been instrumental in analyses of early embryogenesis in decapod crustaceans as summarised in a number of recent reviews [1, 2, 4-7]. The contribution by Harzsch and Viertel [3] provides a number of additional tips and tricks for immunohistochemistry in crayfish embryos which are relevant for working with other decapod larvae, too.

In immunohistochemical experiments, the penetration of large molecules such as antibodies into the tissue to reach their target antigens is an essential process. Dissected tissues from adult specimens can be cut to histological sections to achieve a good penetration by reagents, by for example using a vibratome. For decapod larvae, the manual dissection to remove individual organs such as the central nervous system or midgut gland with fine forceps may be a superior method for tissue preparation, a challenging process that needs much patience, practice, and manual skills. The dissected tissues can then be processed as whole mounts which is advantageous because the 3D organisation of the organs is preserved thus facilitating the microscopic analysis of the specimens. Nevertheless, immunohistochemistry has also successfully been used on histological sections of larvae [135, 136]. To summarise, apart from choosing suitable primary antisera, a solid strategy for tissue preparation is essential when envisaging immunohistochemical experiments.

The primary antisera are visualised by secondary antisera conjugated to certain detection systems [133, 134]. In the 1980s and 1990s, secondary antisera conjugated to enzymes were most popular. These enzymes were the key to a chromogenic reaction which catalysed the polymerisation of a dye as a marker for the primary reagents within the tissue. Such preparations could be viewed by bright field microscopy and were then open to analyses by an acute observer and drawing with a camera lucida apparatus $[137,138]$. As an example, Fig. 16 shows drawings of neuronal elements in the central nervous system of Zoea 1 and 2 of Hyas araneus that were immunolabelled with an antiserum against the neuropeptide FMRFamide. This study revealed a rapid maturation of the neurons expressing this peptide from one stage to the next, especially in the ventral nerve cord [138]. For the last 15 years or so, fluorescent dyes have been the primary choice as conjugates for secondary detection reagents. Secondary antisera conjugated to fluorochromes are essential for double or triple labelling experiments (Fig. 15). However, such preparations cannot be imaged by bright field microscopy but need more expensive fluorescent microscopes for analysis. Confocal laser scanning microscopy today is the state-of-the-art method to analyse immunohistochemical preparations, an imaging technique which allows to explore the third dimension of whole mount tissues by recording Z-stacks of optical sections. The potential of light sheet microscopy to visualize the larval morphology still needs to be explored.

\section{Description of the method}

The first step in any immunohistochemical protocol is a chemical fixation of the larvae (see companion paper [8]). A typical protocol involves immersion in $4 \%$ paraformaldehyde (PFA) in PBS (0.1 M phosphate- buffered saline $\mathrm{pH}$ 7.4) for $4 \mathrm{~h}$ at room temperature. To allow a rapid penetration of the fixative, it is recommended to cut off some of the larval appendages such as spines or limbs as long as these are not the target organs (see companion paper [8]). The choice of the fixative and fixation time depends on the antiserum you plan to use and fixatives other than PFA may be more appropriate [3]. The chemical nature of the antigen that was used to immunise the host animals for generating the antiserum is an essential parameter to know to adjust the fixation protocol, a piece of information that is included in the manufacturer's product sheets that come with the antisera. Because the most essential step in immunohistochemistry is a strong and selective binding of the primary antibody to its antigen, we recommend reading some background information on the biochemical principles of fixation using available text-books to gain background knowledge of the biochemistry of this process (e.g., [133, 134]).

Wash dissected organs in a fresh dish (i.e. it has not been previously in contact with fixative) in six steps of PBS for at least 2-4 $\mathrm{h}$ at room temperature under gentle agitation on an orbital shaker to remove the chemical fixative. You may pause your experiment at the end of the day by leaving the specimens in PBS overnight. In this case, store the dish at $4{ }^{\circ} \mathrm{C}$, and in the last washing step, add $0.02 \%$ sodium-azide. Sodium azide is extremely 

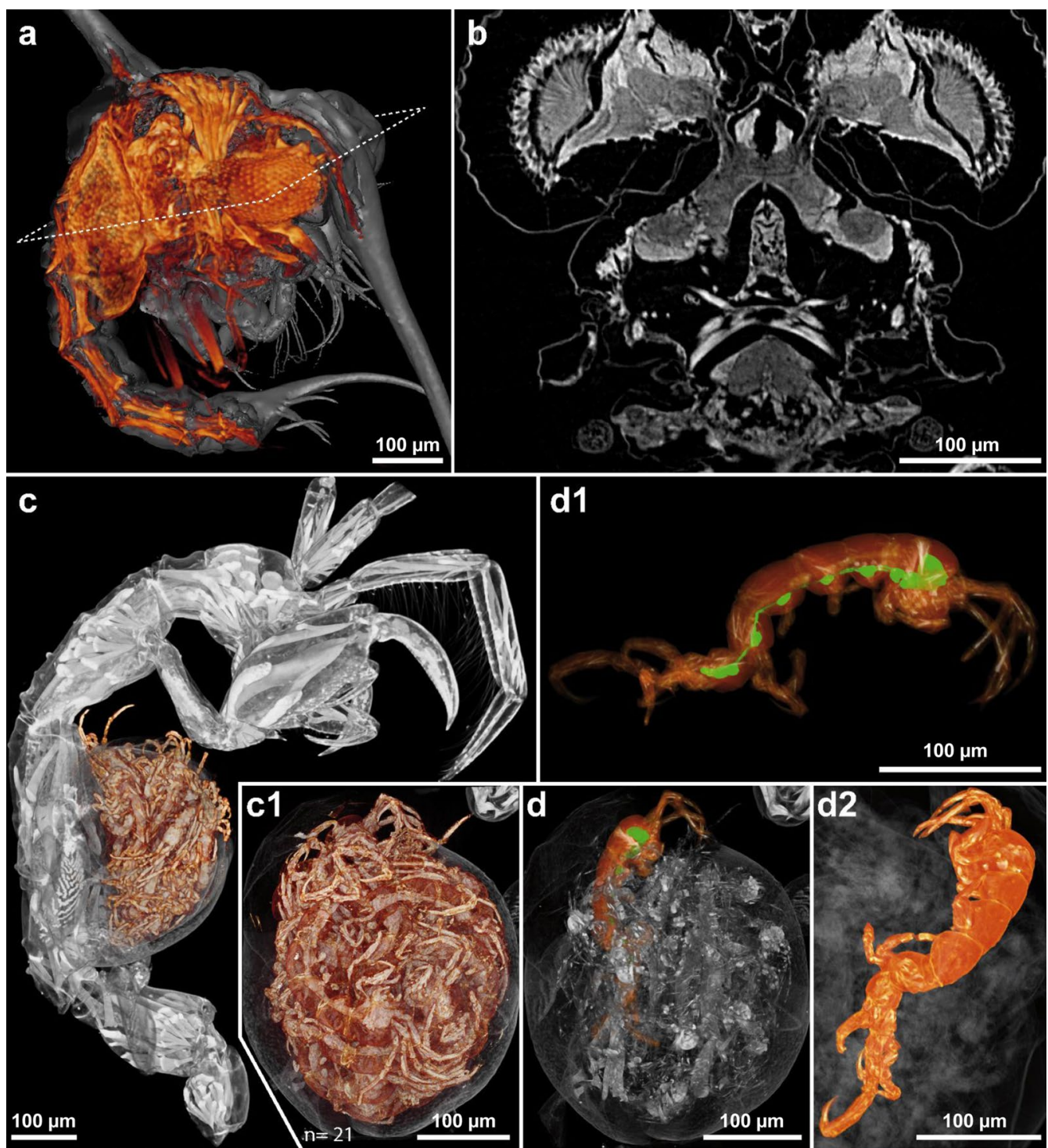

Fig. 12 Visualisations of malacostracan developmental stages based on $\mu C T$ (all images J. Krieger, unpublished; see section "X-ray microscopy $(\mu \mathrm{CT}) "$ for details). a Based on a $\mu \mathrm{CT}$-dataset of a Zoea II of Carcinus maenas, the outer surface (here, virtually sliced along the midline in grey) can be visualised ("Isosurface"-module in Amira ${ }^{\circledR}$; compare Table 2) in addition to a volume rendering ("Volren-module" in Amira ${ }^{\circledR}$ ) showing internal elements of the musculature, stomach as well as the compound eye (orange colourmap). b Two-dimensional virtual slice through that larva along the dorso-ventral body-axis as indicated by the dashed line in a). Note that the space between cuticle and internal elements is an artefact resulting from tissue shrinking due to chemical fixation. Differences in the tissue densities, however, allow the discrimination for manual segmentation of desired organs based on the resulting different greyscales. c $\mu C$ C -scanned female of the amphipod Caprella mutica ("Volume-rendering" in grey) and 21 post-larvae (Manca-stage) located inside the marsupium ("Volume-rendering" in orange based on the manual segmentation of the interior marsupial cavity (in higher magnification in $\mathbf{c 1}$ ). d, d1, d2: one post-larva (orange) was randomly selected and manually segmentated and also its nervous system reconstructed (green in $\mathbf{d}$ and $\mathbf{d} \mathbf{1}$ ). Using manual segmentation, a single juvenile can be either highlighted among its 20 siblings (d), or visualised as isolated individual for detailed anatomical analysis (d1 $\mathbf{1}$ and $\mathbf{d} \mathbf{2}$ )

toxic and serves as an antibiotic to avoid contaminating bacteria growing as you process your samples. Use a dissecting microscope when changing buffers. Figure $17 \mathrm{~d}$ shows some additional tools such as pipettes with drawn out tips and containers recommended for the following steps (see also [3]). Place the transparent dishes on black cardboard to improve visibility of the transparent tissues and use illumination from above. When exchanging the PBS, carefully replace the fluid (but not the target tissues) with a fresh volume of buffer. Cover the well plates with Parafilm ${ }^{\circledR}$ during the washing steps. 
Next, preincubate tissues in PBS-TX (0.1 M PBS, 0.3\% Triton X-100, $1 \%$ bovine serum albumin, $0.02 \%$ sodium azide) for one hour at room temperature with gentle agitation. Triton X-100 serves as a detergent and increases the permeability of the tissue. The bovine serum albumin in this reagent is supposed to cover the tissues with a coat of loosely bound protein. This cover is supposed to reduce the binding of unspecific antibodies in the next step (when using polyclonal antisera). After the preincubation step, immerse tissues overnight in primary antibody in PBS-TX at room temperature. For double labelling experiments, incubate in a cocktail of both primary antisera. The concentration of the primary antibody is an essential parameter and must be determined in pilot experiments. We recommend incubation overnight but longer periods are possible depending on the samples. Cover the dish with Parafilm to avoid contamination and evaporation of the liquid. Refer to [3] for more instructions on this step. Subsequently, wash the samples for at least $2 \mathrm{~h}$ in several changes of PBS at room temperature and gentle agitation.

Next, incubate in secondary antisera (diluted in PBS at the manufacturer's recommendations) for $2-4 \mathrm{~h}$ at room temperature. Too high concentrations of the secondary antisera may increase background labelling. In double labelling experiments, incubation must be carried out in a cocktail of the secondary antisera, for example, antimouse Alexa Fluor $488^{\circledR}$ plus anti-rabbit Cy3 secondary antisera. As a third label, a DNA-marker to label cell nuclei may be added at this stage to visualise the general architecture of the tissues (see section "Specific markers for cell nuclei").

Wash the tissues thoroughly for at least $2 \mathrm{~h}$ in several changes of PBS at room temperature. Longer washing steps help to reduce background labelling. For mounting the specimens on slides and cover-slip them, commercial mounting media such as Gelmount ${ }^{\circledR}$, Vectashield ${ }^{\circledR}$ and others are available which frequently contain "antifade" reagents that are supposed to reduce photo bleaching when viewing the preps. For short time preservation, plain glycerol can be used, a medium that does not polymerise. Mowiol ${ }^{\circledR}$-Glycerol is another convenient alternative for mounting specimens.

\section{Additional comments}

Because of the availability of hundreds if not thousands of commercially available antisera, immunohistochemistry potentially is a powerful technique to study developmental processes in crustaceans. However, working with non-model systems means that specificity of the reagents is an important issue and that complex control experiments are essential to show that the chosen antisera do label what they should label. What is more, the necessary reagents are relatively expensive (see Table 1), at least in the line-up of techniques that we described here. Although the samples can be visualised with conventional fluorescence microscopy, exploring the full potential of this approach requires confocal laser scanning microscopy and hence an expensive optical instrument.

\section{Using an in vivo marker for cell proliferation Introduction}

Organogenesis during larval growth is characterised by a high level of mitotic cell proliferation. Markers for certain phases of the cell cycle are suited to locate mitotic cells in the tissues and to analyse the temporal dynamics of cell proliferation (Fig. 17). The thymidine analogon 5-bromo-2-deoxyuridine (BrdU) is a wellknown $\mathrm{S}$ phase-specific marker to analyse neurogenesis in adult crustacean brains [139, 140, 140-143]. This marker was also applied to study aspects of neurogenesis in late crustacean embryos and larvae such as the development of the ventral nerve cord [144-146], the brain [147-149] and the compound eyes [150-152]. Larvae are incubated in vivo in the labelling reagent diluted in sea water for $2-10 \mathrm{~h}$, during which the marker is taken up into the organisms where it gets incorporated into single-stranded DNA during S-phase in all cell nuclei which are cycling during the incubation period. Therefore, strictly speaking, this is not a mitosis marker but a replication marker. After fixation of the animals, the marker is visualised by immunohistochemistry. Because the marker gets distributed across the entire organism, it allows the localisation of cycling cell nuclei in all organs of the larvae. Depending on how long you incubate the larvae, you may study cell cycle dynamics in the target organs. In so-called "pulsechase" experiments, larvae are transferred back to normal seawater after incubation in the marker where they continue to develop. By sacrificing cohorts of larvae at different time points after the BrdU pulse, additional aspects of cell cycle dynamics can be studied.

\section{Description of the method}

In vivo labelling For labelling, decapod larvae are incubated in vivo in the liquid BrdU-labelling reagent RPN 201 (Sigma-Aldrich, GE Healthcare) diluted with filtered seawater (32 PSU) to a final concentration of $0.2 \mathrm{mg} / \mathrm{ml}$ (after [149]). We determined an incubation period of $6 \mathrm{~h}$ at a typical rearing temperature of $12-15{ }^{\circ} \mathrm{C}$ as ideal for labelling cycling cells in the developing brain and ventral nerve cord of Carcinus maenas (a brachyuran crab) larvae (Fig. 17). However, studies of other target organs may 

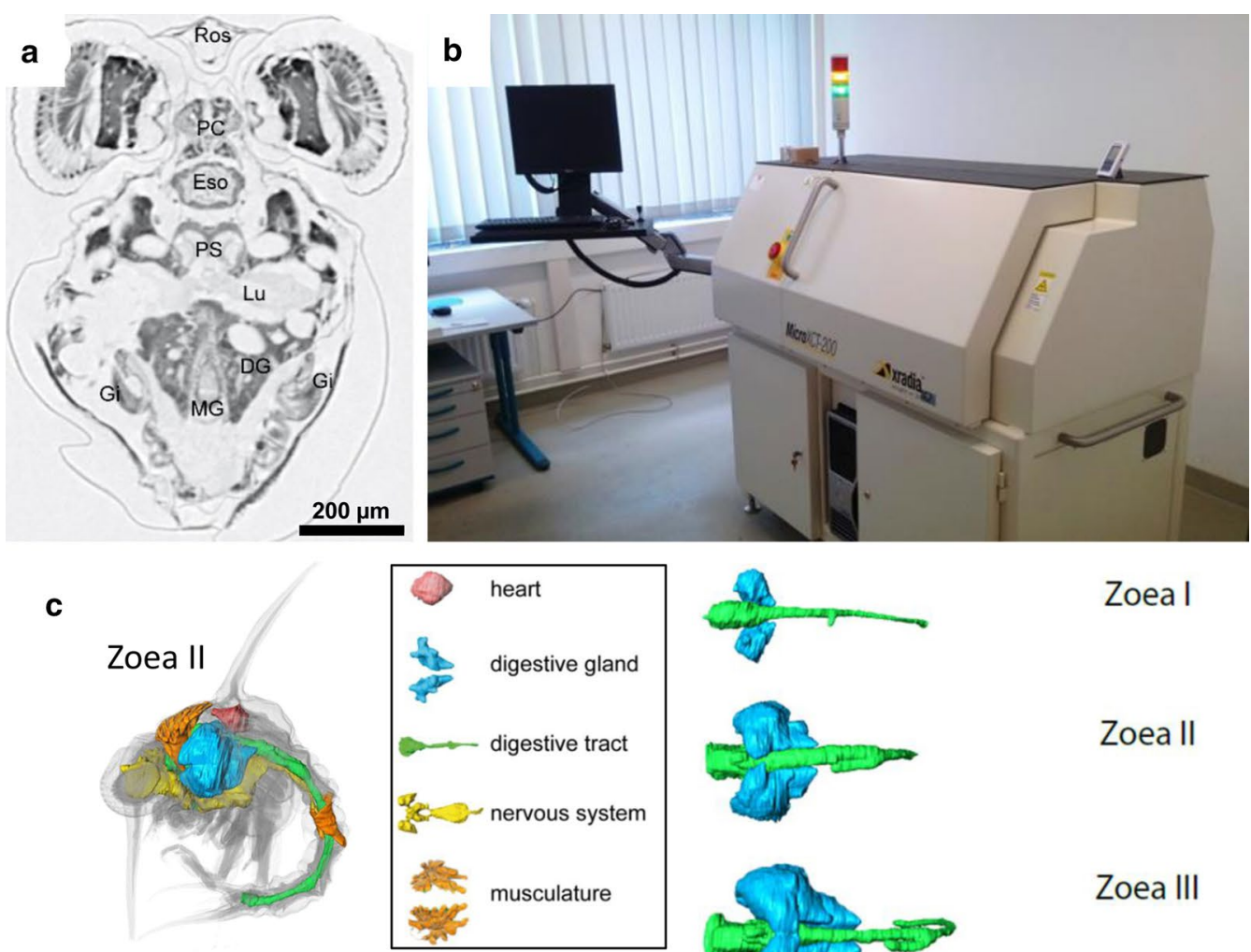

Zoea I

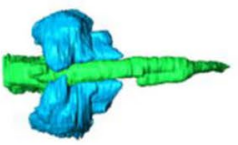

Zoea II
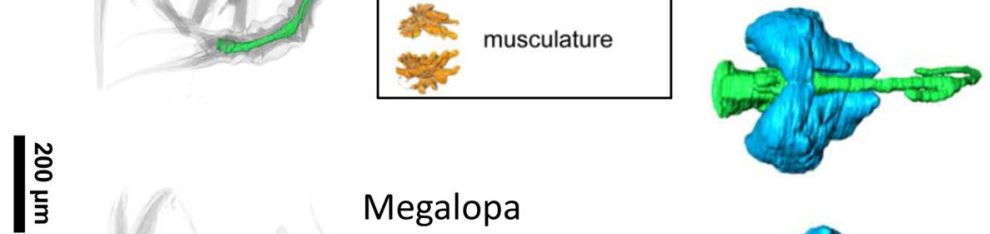

Zoea III
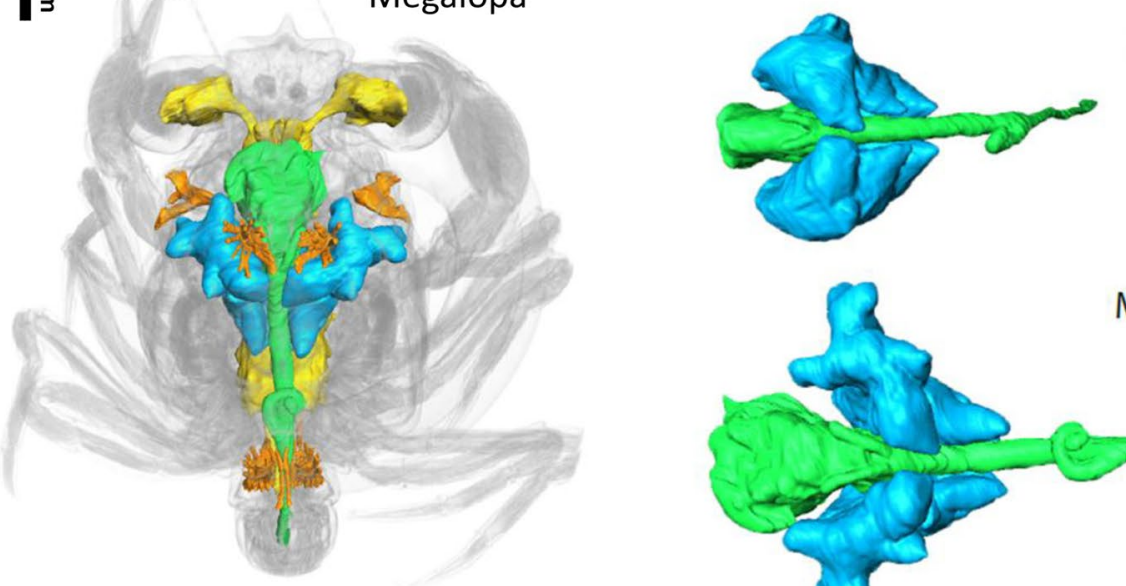

Zoea IV

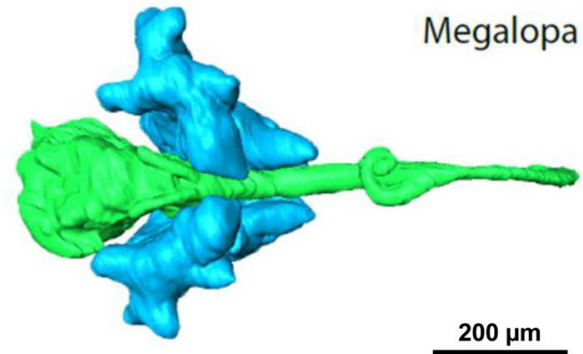

Fig. 13 X-ray microscopy $(\mu \mathrm{CT}$ ) of Carcinus maenas larvae (see $[22,122]$, and section "X-ray microscopy $(\mu \mathrm{CT})$ " for details). a Zoea IV, single horizontal section from $\mu$ CT analysis, black-white inverted (F. Spitzner, unpublished). b Micro-Computertomograph XRadia MicroXCT-200. c 3D reconstructions (Amira) of selected organ systems of C. maenas larvae based on X-ray data (modified from [22]). DG: digestive gland; Eso: (0) esophagus; Gi: gill, Lu: lumen of digestive gland; MG: midgut; PC: protocerebrum; PS: pyloric stomach; Ros: rostrum

demand a different pulse length. In order to control for circadian effect of cell cycle length in quantitative studies, the incubation should always be carried out at the same time of the day. Furthermore, we made sure that all indi- viduals were incubated two days after moulting to avoid interference of the moult cycle. After the BrdU pulse, the larvae are fixed in 4\% PFA (paraformaldehyde) in $0.1 \mathrm{M}$ $\mathrm{PBS}$ and stored at $4{ }^{\circ} \mathrm{C}$ until further processing. 

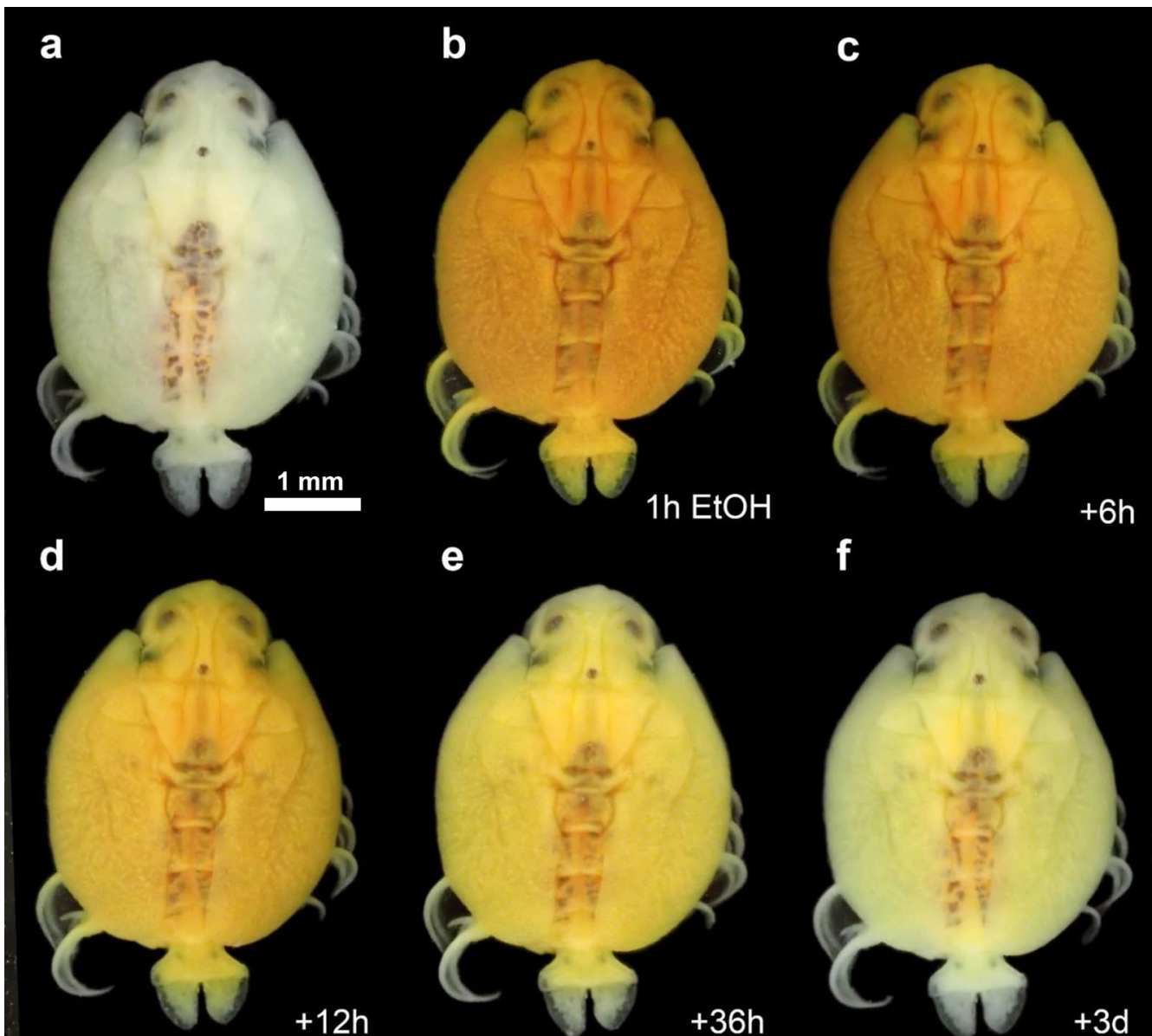

$\theta$

f

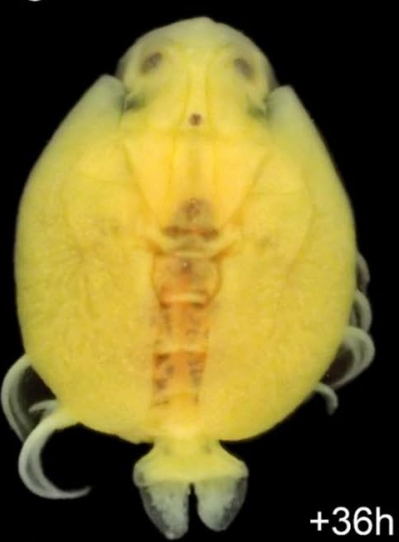

$+6 h$

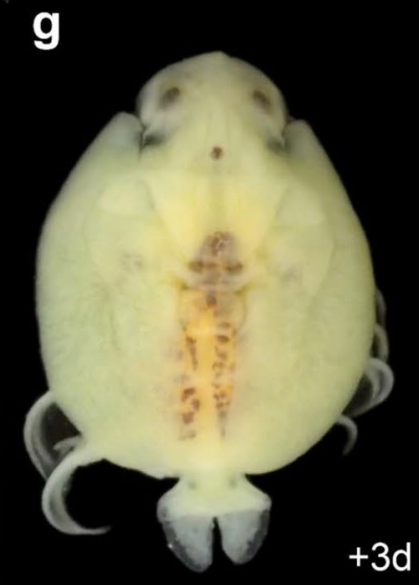

h

$+36 \mathrm{~h}$
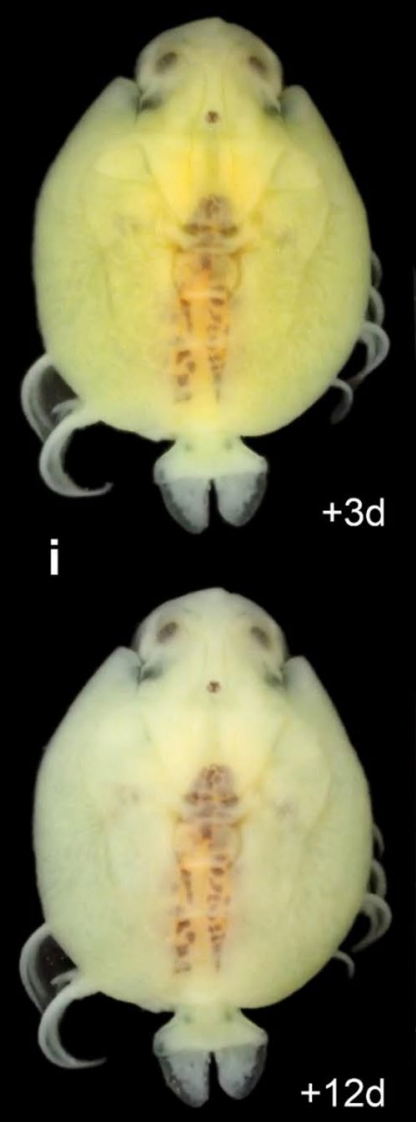

Fig. 14 Argulus foliacaeus stained using a 2\% iodine solution for $\mu \mathrm{CT}$ sample preparation to show the gradient of decolouration after several washing steps (Hörnig, unpublished; see section "X-ray microscopy ( $\mu \mathrm{CT}$ )" for details). a Unstained, b-i Eight washing steps in $99.8 \%$ ethanol during ca. 23 days in total (cumulative timescale), b after 1 h, c after 6 h, $\mathbf{d}$ after 12 h, e after 36 h, $\mathbf{f}$ after 3 days, $\mathbf{g}$ after 3 days, $\mathbf{h}$ after 3 days, i after 12 days. All images show the same individual

Immunohistochemical processing Brains and ventral nerve cords of all stages are dissected using the tools and techniques laid out in the companion paper [8], and processed as whole mounts to better visualise the nuclei in s-phase in a three-dimensional context. To wash out the fixative, the tissues are incubated for at least 

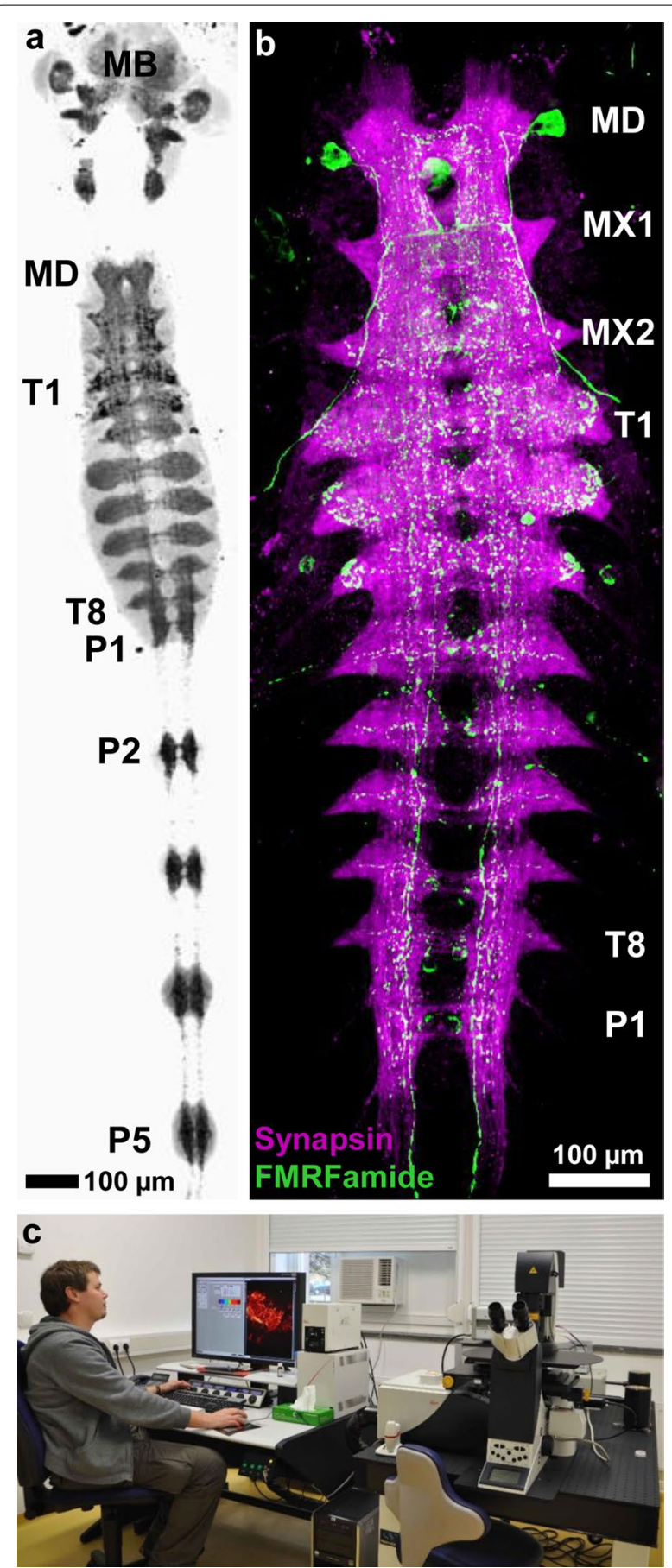

Fig. 15 Immunohistochemical analysis of dissected whole mounts of the central nervous system from larvae of Birgus latro (see section "Immunohistochemistry" for details). a Dissected central nervous system of a Zoea 4 labelled with an antiserum against synaptic proteins to visualise the neuropil areas (T. Kirchhoff, unpublished), black-white inverted confocal laser-scan image. b Double labelling of a ventral nerve cord of a Zoea 2 to show areas of synaptic neuropil (magenta) and FMRFamide-like neuropeptides (green; T. Kirchhoff, unpublished). c Confocal laser-scan microscope Leica SP5 (image S. Harzsch). MD: neuromere of mandible, MX 1, 2: neuromeres of maxilla 1 and 2,T1, 8: thoracic neuromeres 1, 8, P1, P5: pleomeres 1, 5
$3 \times 5$ min in phosphate- buffered saline (PBS). Afterwards, the samples are incubated in $2 \mathrm{M} \mathrm{HCl}$ for $30 \mathrm{~min}$ to denature the DNA and improve the access of the primary antibody against BrdU. This is a harsh but essential step because commercially available nucleases will not accomplish the job. Next, the tissues are incubated for $1.5 \mathrm{~h}$ in PBS-TX (PBS $+0.3 \%$ Triton $\mathrm{X}-100+1 \%$ bovine serum albumine) to further improve antibody penetration. This step was followed by incubation in the primary antisera for $4 \mathrm{~h}$. In order to visualise morphological landmarks in addition to the s-phase nuclei, double labelling experiments can be carried out. For the nervous system, immunohistochemistry against general cytoskeletal elements such as tubulin can provide a good overview of the structure of the developing brain (see [3] for more background on this topic). Therefore, we combined the incubation in the primary anti-BrdU antiserum (rat, 1:1,000) with either an anti-acetylated tubulin antibody (mouse, 1:1,000) or anti-tyrosinated tubulin antibody (mouse, 1:1,000). After the incubation, all tissues are washed in several changes of PBT for $1 \mathrm{~h}$ and incubated in a cocktail of secondary antibodies conjugated to Alexa Fluor $488^{\circledR}$ (goat, anti-rat, 1:500) and Cy $3{ }^{\circledR}$ (goat, anti-mouse, 1:500). As an option, the DNA marker HOECHST ${ }^{\circledR}$ 33,342 (Thermo Fisher Scientific; H3570) can be used as a nuclear marker as a third label to visualise the neuronal cell clusters during this step (see section Immunohistochemistry). Finally, the tissues are washed in several changes of PBT for $3 \mathrm{~h}$. After the last wash in PBT, the brains are transferred to a 1:1 glycerol:PBS mixture for 20 min followed by a further 60 min incubation in a 4:1 glycerol-PBS mixture. Finally, the tissues are mounted in a 4:1 DABCO-glycerol:PBS mixture. For tips and tricks concerning mounting small items such as dissected brains, see[3]. Whole mount brains are scanned using a Leica TCS SP5II confocal laser scanning microscope using DPSS (561 nm), diode $(405 \mathrm{~nm})$ and argon $(488 \mathrm{~nm})$ lasers. The software Leica Application Suite Advanced Fluorescence (LASAF) is used to operate the microscope. The initial evaluation of the data can be done using the LASAF Lite software. Furthermore, the open source software FIJI [153], is recomended to generate maximum projections of substacks of some Z-stacks (see Table 2). The depth encodings shown in Fig. 17a were created using the " $Z$ code stack" plugin for ImageJ.

\section{Additional comments}

BrdU-labelling has turned out a robust technique which can be used as a tool to analyse cell proliferation from crustacean embryos (review $[1,2]$ ) across the larvae to adult animals [139, 140, 140-143]. However, when interpreting the results of the method presented here it has 
to be borne in mind that this technique labels DNA synthesis which is not necessarily equivalent to mitosis considering that cells may replicate their DNA without cell division to become polyploid. Other markers for different phases of the cell cycle need to be established as tools to study organogenesis in crustacean larvae. Another disadvantage is that it is difficult to determine which cell type the labelled nucleus belongs to. In the nervous system for example, we know that in addition to neuronal stem cells, also the nuclei of glial cells may replicate as well as those of blood vessels in the nervous tissue. This set-back can be overcome by long-term pulse-chase experiments in which the animals are reared for days or weeks after the BrdU pulse until the labelled cells have differentiated. Double labelling experiments with cell type-specific markers may then reveal the identity of the cells which originated from the cell division while they were pulsed with BrdU.

\section{Conclusions and outlook}

In this paper, we have provided a survey of methods useful to study morphogenesis and organogenesis in decapod larvae but so far have omitted molecular approaches such as in situ hybridisation to localise mRNA. In other words, we have given a survey of classical morphological approaches resulting in images of structures and stagespecific shapes both on cellular or organ level. Such methods must always serve a scientific question, no matter how tempting it might be just to generate attractive images. Therefore, we recommend to first develop a scientific question (some of which were summarised in the introduction) and then to select suitable methods that help to answer the questions and not the other way around. Having said that, the availability of the larvae is essential. When studying larvae reared in the lab (see companion paper [8]) more sophisticated fixation and preparation techniques can be used than in a study of rare material in the field or from museums.

Along these lines, newly established methods are not an end unto themselves. New methods should not be seen as just a tool to repeat something that has already been analysed before with a new method to eventually generate better images than the older techniques. Rather, new techniques should open new research avenues. New techniques may provide new insights into processes of morphogenesis and-by the comparative approach the evolution of developmental processes, an approach which has been called EvoDevo or MorphoEvoDevo for many years now. As shown in Table 1, there are methods for various budgets, some of which need no more than a microscope or stereomicroscope, a staining solution and of course larvae, some will need quite an investment before you can apply them, like SEM, TEM, and $\mu$ CT.

We wish to communicate one handy hint here: watch the living animals carefully before you start your experiment. Laboratory cultures as described in the companion paper [8] are an ideal starting point for such observations as are larvae caught with a plankton net. Larvae, alive, in a little aquarium or a rearing bowl, feeding on Artemia nauplii, are fascinating creatures. They are tiny-but they are able to do so much and so complex is their behaviour. Morphologists recommend that a study should always begin with observing the living animal, its movement back and forth, up and down, its way of feeding, its resting posture, etc. The more you know about your study object, the better the preparation and fixation will be, and only after patient observation, you will find the best way of placing the larvae under your imaging device. Use a little pocket lamp and light a corner of your aquarium. If phototactic, the larvae will immediately swim to the bright spot as they would also do in the ocean. Put some of them in a little vial and study them under the microscope before using any complicated techniques.

We have another important suggestion: play and try uncommon things. There is always a way around mediocre fixation, poor embedding, shrunken tissues-also for non-model organisms. Some of the fixations shown in the companion paper [8] have been carried out under the simplest field conditions. If necessary, a sugar bag from a coffee house at some Mediterranean harbour can be the beginning of a series of fixations of different osmolarities that produce the high-quality results you are looking for.

A substantial progress in the last two decades has been the development of 3D reconstruction methods (Figs. 9, 13), either based on images taken from serial sections or on imaging devices that produce perfectly aligned images per se, such as micro-computed X-ray tomography, confocal

(See figure on next page.)

Fig. 16 Immunohistochemical localisation of FMRFamide-like neuropeptides in whole mounts of the central nervous system of zoeal stages of Hyas araneus (modified from [138]; see section "Immunohistochemistry" for details). The primary antiserum was visualised with an enzyme-based detection system and the preparations analysed with a bright field microscope (Polyvar, Reichert Jung) and drawn using an attached camera-lucida apparatus to thoroughly analyse the maturation of single neuronal elements AMC: anterior median cell cluster; APN: anterior protocerebral neuropil; CG: commissural ganglion; MD: neuromere of mandible; MX 1, 2: neuromeres of maxilla 1 and 2; T1, 8: thoracic neuromeres 1, 8; P1, P5: pleomeres 1, 5; PPN: posterior protocerebral neuropil; O: oesophageal foramen; OC: oesophageal connective; OL: olfactory lobe; POC: postoesophageal commissure; TCN: tritocerebral neuropil 


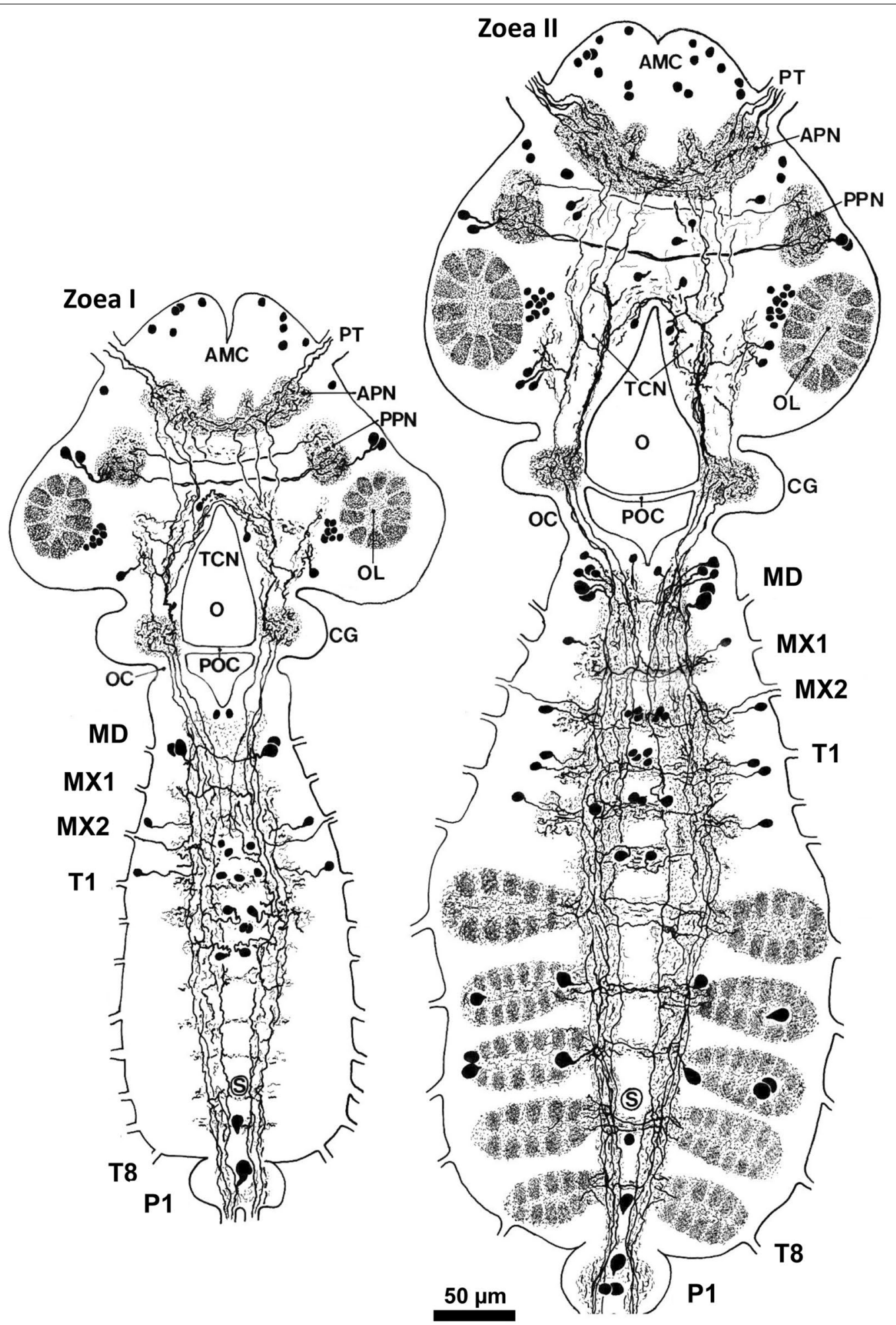




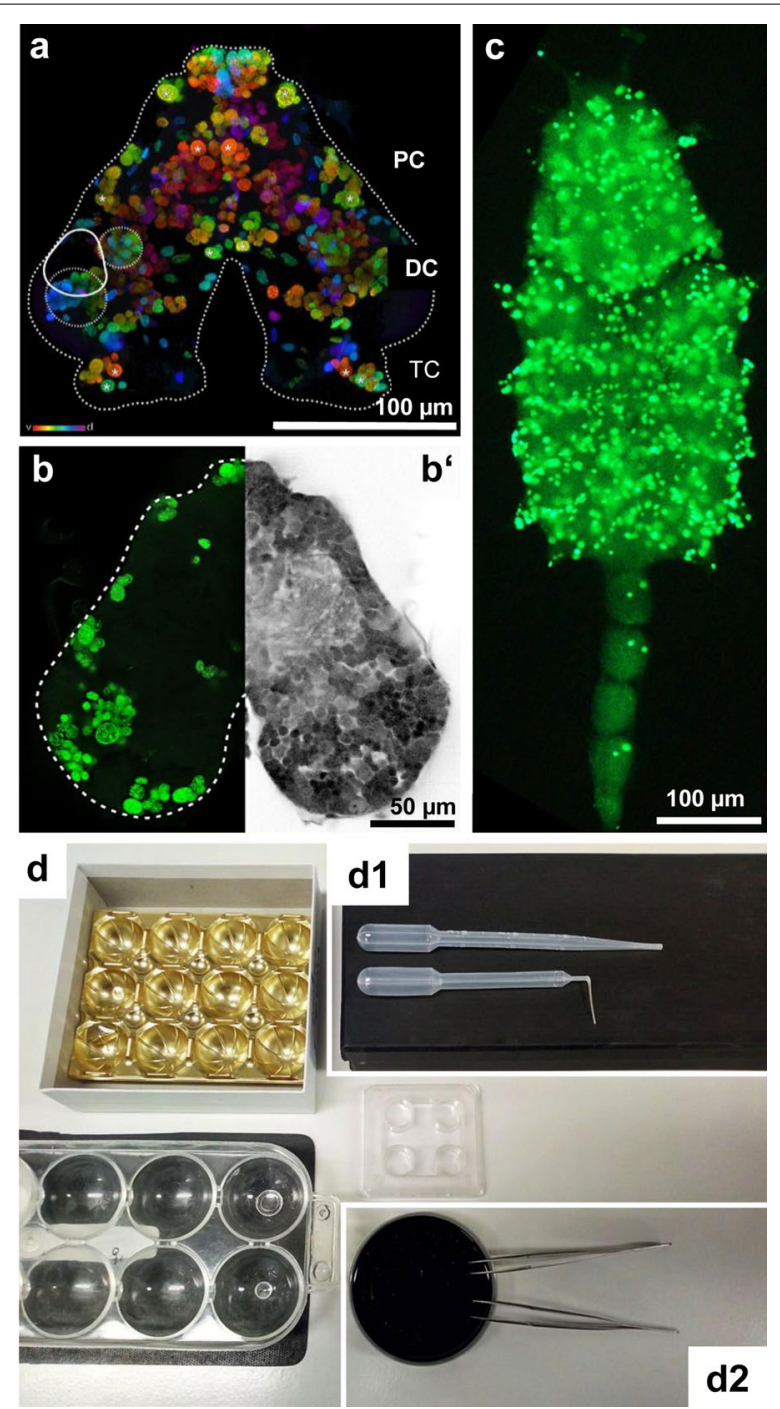

Fig. 17 BrdU labelling of cell nuclei in s-phase in dissected whole mounts of the median brain and ventral nerve cord of $C$. maenas larvae scanned using a Leica TCS SP5II confocal laser scanning microscope (see section "Using an in vivo marker for cell proliferation" for details). a Median brain, coloured depth coding using the "Z code stack" plugin for ImageJ (R. Meth, unpublished). b Dorsal surface view of a Zoea III median brain (R. Meth, unpublished). b' Autofluorescence of the right brain hemisphere visualised by UV excitation (R. Meth, unpublished). c Ventral nerve cord of a Megalopa imaged with a Nikon Eclipse 90i upright fluorescence microscope (S. Harzsch, unpublished). $\mathbf{d}$ Various containers for immunohistochemistry (image S. Harzsch). d1 Plastic pipettes with drawn-out tips (image S. Harzsch). d2 Petri dish with black Elastosil ${ }^{\circledR}$ coating and micro-forceps. PC: protocerebrum; DC: deutocerebrum; TC: tritocerebrum are, and sometimes also the changes of morphology with time, with developmental stages, allowing for 4D analyses. Moreover, we are sure that we are not at the end of staining tricks for all kinds of tissues and cells. We have shown some, but there are many more, and also some we don't know yet. Hence, try something new, but also use old tricks that otherwise would be forgotten. Try, for example, methysalicylate, oil of wintergreen, to clear larvae under the microscope, and maybe see things you have never been able to see before $[72,154]$. Then, switch on the epifluorescence-wintergreen oil increases the autofluorescence of the cuticle in a quite interesting way [155]. Furthermore, the latest aqueous tissue clearing methods will surely open new avenues to the study of larval morphology [156]. And there is much more to try, much more to dig out of almost forgotten publications about classical preservation and histological methods, and of publications about other taxa that have never been tried with decapod larvae. Good luck!

\section{Acknowledgements}

We dedicate this review to Mireille and Guy Charmantier, two of the pioneers in analysing organogenesis in decapod crustacean larvae. SH gratefully acknowledges the hospitality of the Biologische Anstalt Helgoland, AlfredWegener-Institut, Helmholtz-Zentrum für Polar- und Meeresforschung, during the final phase of writing this manuscript. We wish to thank Gabriele Uhl and Shou-Wang Lin (Greifswald) for the collaboration in the experiments shown in Fig. 14. Grant numbers: DFG INST 292/119-1 FUGG, DFG INST 292/120-1 FUGG. FS, ZS, and SH were supported by DFG Research training Group 2010 RESPONSE JTH is currently funded by the Volkswagen Foundation with a Lichtenberg professorship.

\section{Authors' contributions}

RM and SH conceived and wrote this paper. SH compiled the plates. FS, ZS, $M K H, J K, C H, J H, T K, R M$ and GT all contributed major parts of the text, assisted in writing the paper and contributed images. All authors read and approved the final manuscript.

\section{Funding}

Open Access funding enabled and organised by Projekt DEAL. FS, ZS, and SH were supported by the DFG Research training Group 2010 RESPONSE. Additional funding: DFG INST 292/119-1 FUGG, DFG INST 292/120-1 FUGG.

\section{Availability of data and materials}

Not applicable because we do not report any original data here.

\section{Declarations}

Ethics approval and consent to participate

Not applicable because this technical review does not report any original experiments.

\section{Consent for publication}

Not applicable.

\section{Competing interests}

none.

\section{Author details}

${ }^{1}$ Bavarian State Collection of Zoology - SNSB, Münchhausenstraße 21, 81247 Munich, Germany. ${ }^{2}$ Department of Biology II, LMU Munich, Biocenter, Großhaderner Str. 2, Martinsried, 82152 Planegg, Germany. ${ }^{3}$ GeoBio-Center der LMU München, Richard-Wagner-Str. 10, 80333 Munich, Germany. ${ }^{4}$ Department of Cytology and Evolutionary Biology, Zoological Institute and Museum, University of Greifswald, Greifswald, Germany. ${ }^{5}$ Biologische Anstalt Helgoland, also a "Heidelberger Hobel" (serial block-face SEM) or "focused ion beam" (FIB-SEM) device in combination with a field emission SEM. This has allowed us to study morphologies in their full three-dimensionality as they really 
Alfred-Wegener-Institut, Helmholtz-Zentrum für Polar- und Meeresforschung Helgoland, Germany.

\section{Received: 4 October 2020 Accepted: 10 May 2021}

Published online: 02 June 2021

\section{References}

1. Loose G, Vogt G, Charmantier-Daures M, Charmantier G, Harzsch S. Organogenesis. In: Anger K, Harzsch S, Thiel M, editors. The Natural History of the Crustacea, Volume 7: Developmental Biology and Larval Ecology. Oxford University Press; 2020. p. 79-111.

2. Harzsch S, Krieger J, Faulkes Z. "Crustacea": Decapoda - Astacida. In: Wanninger A, editor. Evolutionary Developmental Biology of Invertebrates 4: Ecdysozoa II: Crustacea. Wien: Springer Verlag; 2015. p. 101-52.

3. Harzsch S, Viertel C. Immunolocalization of neurotransmitters and neuromodulators in the developing crayfish brain. In: Sprecher SG, editor. Brain development: methods and protocols. New York: Springer; 2020. p. 271-91.

4. Scholtz G. From a single cell to segmental structures: crustacean embryology. In: Anger K, Harzsch S, Thiel M, editors. The Natural History of the Crustacea, Volume 7: Developmental Biology and Larval Ecology. Oxford University Press; 2020. (in press).

5. Scholtz G, Wolff C. Arthropod embryology: cleavage and germ band development. In: Minelli A, Boxshall G, Fusco G, editors. Arthropod biology and evolution. Berlin, Heidelberg: Springer; 2013. p. 63-89.

6. Hertzler PL. "Crustacea": Decapoda Dendrobranchiata. In: Wanninger A, editor. Evolutionary developmental biology of invertebrates 4: Ecdysozoa II: Crustacea. Wien: Springer Verlag; 2015. p. 63-100.

7. Wolff C, Gerberding M. Crustacea: comparative aspects of early development. In: Wanninger A, editor. Evolutionary developmental biology of invertebrates 4: Ecdysozoa II: Crustacea. Wien: Springer Verlag; 2015. p. 39-62.

8. Torres G, Melzer R, Spitzner F, Sargac Z, Harzsch S, Gimenez L. Methods to study organogenesis in decapod crustacean larvae I: larval rearing, preparation and fixation. Helgoland Mar Res. 2021. (in press).

9. Torres G, Spitzner F, Harzsch S, Giménez L. Ecological Developmental Biology and global ocean change: brachyuran crustacean larvae as models. In: Perspectives on evolutionary (and) developmental biology. Padova University Press; 2018. p. 283-306.

10. Anger K. Contributions of larval biology to crustacean research: a review. Inv Reproduct Dev. 2006;49:175-205.

11. Anger K. The biology of Decapod Crustacean Larvae. 1st ed. Lisse: A.A. Balkema Publishers; 2001.

12. Möller OS, Anger K, Guerao G. Patterns of larval development. In: Anger K, Harzsch S, Thiel M editors: The Natural History of the Crustacea Volume 7: Developmental Biology and Larval Ecology. Oxford University Press; 2020. p. 165-94

13. Olesen J. Crustacean life cycles-developmental strategies and environmental adaptations. In: Anger K, Harzsch S, Thiel M editors. The Natural History of The Crustacea Volume 5: Life Histories. Oxford University Press; 2018. p. 1-34

14. Haug JT, Haug C. "Crustacea": comparative aspects of larval development. In: Wanninger A, editor. Evolutionary developmental biology of invertebrates 4. Vienna: Springer; 2015. p. 63-100.

15. Anger K, Queiroga H, Calado R. Larval development and behaviour strategies in Brachyura. In: Castro P, Davie PJF, Guinot D, Schram FR, von Vaupel Klein JC, editors: Treatise on Zoology-Anatomy, Taxonomy, Biology. The Crustacea, Vol. 9, part C-I, Decapoda: Brachyura. Leiden, Boston: Brill; 2015. p. 317-74.

16. Cohen JH, Epifanio CE. Response to Visual, Chemical, and Tactile Stimuli. In: Anger K, Harzsch S, Thiel M editors: The Natural History of the Crustacea Volume 7: Developmental Biology and Larval Ecology. Oxford University Press; 2020. p. 332-59.

17. Epifanio $\mathrm{CE}$, Cohen JH. Behavioural adaptations in larvae of brachyuran crabs: A review. J Exp Mar Bio Ecol. 2016;482:85-105.

18. Gebauer P, Giménez L, Hinojosy I, Paschke K. Settlement and Metamorphosis in Barnacles and Decapods. In: Anger K, Harzsch S, Thiel M editors: The Natural History of the Crustacea Volume 7: Developmental Biology and Larval Ecology. Oxford University Press; 2020. p. 223-53.
19. Forward RB. Larval Biology of the Crab Rhithropanopeus harrisii (Gould): A Synthesis. Biol Bull. 2009;216:243-56.

20. Forward RB, Tankersley RA, Rittschof D. Cues for Metamorphosis of Brachyuran Crabs: An Overview. Integr Comp Biol. 2001;41:1108-22.

21. Haug JT. Metamorphosis in Crustaceans. In: Anger K, Harzsch S, Thiel M editors: The Natural History of the Crustacea Volume 7: Developmental Biology and Larval Ecology. Oxford University Press; 2020. p. 254-83.

22. Spitzner F, Meth R, Krüger C, Nischik E, Eiler S, Sombke A, et al. An atlas of larval organogenesis in the European shore crab Carcinus maenas L. (Decapoda, Brachyura, Portunidae). Frontiers in Zoology. 2018;15:27.

23. Castejón D, Rotllant G, Alba-Tercedor J, Font-i-Furnols M, Ribes E, Durfort $\mathrm{M}$, et al. Morphology and ultrastructure of the midgut gland ("hepatopancreas") during ontogeny in the common spider crab Maja brachydactyla Balss, 1922 (Brachyura, Majidae). Arthropod Struct Dev. 2019;49:137-51

24. Castejón D, Ribes E, Durfort M, Rotllant G, Guerao G. Foregut morphology and ontogeny of the mud crab Dyspanopeus sayi (Smith, 1869) (Decapoda, Brachyura, Panopeidae). Arthropod Struct Dev. 2015;44:33-41.

25. Castejón D, Alba-Tercedor J, Rotllant G, Ribes E, Durfort M, Guerao G. Micro-computed tomography and histology to explore internal morphology in decapod larvae. Sci Rep. 2018;8:14399.

26. Haug JT, Martin JW, Haug C. A 150-million-year-old crab larva and its implications for the early rise of brachyuran crabs. Nature Comm. 2015:6:6417.

27. Haug C, Mayer G, Kutschera V, Waloszek D, Maas A, Haug JT. Imaging and Documenting Gammarideans. Int J Zool. 2011.

28. Haug JT, Haug C, Ehrlich M. First fossil stomatopod larva ( Arthropoda Crustacea ) and a new way of documenting Solnhofen fossils ( Upper Jurassic, Southern Germany ). Palaeodiversity. 2008;:103-9.

29. Haug JT, Labandeira CC, Santiago-Blay JA, Haug C, Brown S. Life habits, hox genes, and affinities of a 311 million-year-old holometabolan larva. BMC Evol Biol. 2015:15:208.

30. Eiler SM, Haug C, Haug JT. Detailed description of a giant polychelidan eryoneicus-type larva with modern imaging techniques. Spixiana. 39:39-60.

31. Nagler C, Høeg JT, Haug C, Haug JT. A possible 150 million years old cirripede crustacean nauplius and the phenomenon of giant larvae. Contribut Zool. 2017;86:213-27.

32. Haug JT, Haug C, Kutschera V, Mayer G, Maas A, Liebau S, et al. Autofluorescence imaging, an excellent tool for comparative morphology. J Microsc. 2011:244:259-72.

33. Tanković MS, Baričević A, Perusco VS, Melzer RR, Lopez Al, Dömel JS, et al. Experimental evidence for shaping and bloom inducing effects of decapod larvae of Xantho poressa (Olivi, 1792) on marine phytoplankton. J Mar Biol Ass. 2018;98:1881-7.

34. Zupo V, Buttino I. Larval development of decapod crustaceans investigated by confocal microscopy: an application to Hippolyte inermis (Natantia). Mar Biol. 2001;138:965-73.

35. Haug JT, Haug C. Fossilien unter langwelligem Licht: Grün-Orange-Fluoreszenz an makroskopischen Objekten. Archaeopteryx. 2011;29:20-3.

36. Haug C, Shannon KR. Isolated mantis shrimp dactyli from the Pliocene of North Carolina and their bearing on the history of Stomatopoda. Boletín de la Sociedad Geológica Mexicana. 2013;65:273-84.

37. Haug JT, Haug C, Maas A, Fayers SR, Trewin NH, Waloszek D. Simple 3D images from fossil and Recent micromaterial using light microscopy. J Microsc. 2009;233:93-101.

38. Reimer L, Pfefferkorn G. Raster-Elektronenmikroskopie. 2nd edition. Berlin Heidelberg: Springer-Verlag; 1977.https://doi.org/10.1007/ 978-3-642-81112-8.

39. Melzer RR, Panzinger A, Reckel F, Smola U. Central nervous system of brachyceran larvae (Insecta, Diptera). Zool Anz. 1995:234:113-24.

40. Tanaka K. Scanning Electron Microscopy of Intracellular Structures. In: Bourne GH, Danielli JF, Jeon KW, editors. International Review of Cytology. Academic Press; 1980. p. 97-125. https://doi.org/10.1016/S00747696(08)62308-6.

41. Meyer R, Melzer RR. Scanning EM diagnosis of marine Decapoda larvae: a comparison of preparation techniques. Crustaceana. 2004;77:883-6.

42. Meyer R, Wehrtmann IS, Melzer RR. Morphology of the first zoeal stage of Portunus acuminatus, Stimpson, 1871 (Decapoda: Portunidae: Portuninae) reared in the laboratory. Sci Mar. 2006:70:261-70. 
43. Meyer R, Lehmann T, Melzer RR, Geiselbrecht H. Morphology of the first zoeal stage of the Mediterranean bumblebee shrimp Gnathophyllum elegans studied with both light microscopy and scanning EM. J Mar Biol Assoc UK. 2014;94:151-8.

44. Geiselbrecht $H$, Melzer RR. Mandibles of zoea I larvae of nine decapod species: a scanning EM analysis. Spixiana. 2010;33:27-47.

45. Meyer R, Friedrich S, Melzer RR. Xantho poressa (Olivi, 1792) and Xantho pilipes A. Milne-Edwards, 1867 Larvae Larvae (Brachyura, Xanthidae): Scanning Em Diagnosis of Zoea I from the Adriatic Sea. Crustaceana. 2004;77:997-1005.

46. Batel A, Melzer RR, Anger K, Geiselbrecht H. Heterochrony in mandible development - A comparative SEM study on zoea larvae of two carideans (Decapoda). J Morphol. 2014;275:1258-72.

47. Krapp T, Lang C, Libertini A, Melzer RR. Caprella scaura Templeton, 1836 sensu lato (Amphipoda: Caprellidae) in the Mediterranean. Org Divers Evol. 2006;6:77-81.

48. Lang C, Krapp T, Melzer RR. Postembryonal development in Caprellidae: SEM description and comparison of ready-to-hatch juveniles and adults of two Mediterranean skeleton shrimps (Crustacea: Amphipoda). Zootaxa. 2007:1605:1-32.

49. Becker C, Dick JTA, Cunningham EM, Schmitt C, Sigwart JD. The crustacean cuticle does not record chronological age: New evidence from the gastric mill ossicles. Arthropod Struct Dev. 2018;47:498-512.

50. Tanaka K, Mitsushima A. A preparation method for observing intracellular structures by scanning electron microscopy. J Microsc. 1984;133:213-22.

51. MacDonald JA, Fowle WH, Woods PhD DC. New insights on mitochondrial heterogeneity observed in prepared mitochondrial samples following a method for freeze-fracture and scanning electron microscopy. Micron. 2017;101:25-31.

52. Boyde A. Review of basic preparation techniques for biological scanning electron microscopy. Electron Microsc Electron Microsc. 1980;2:768-77.

53. Nation JL. A new method using hexamethyldisilazane for preparation of soft insect tissues for scanning electron microscopy. Stain Technol. 1983;58:347-51.

54. Braet F, Zanger RD, Wisse E. Drying cells for SEM, AFM and TEM by hexamethyldisilazane: a study on hepatic endothelial cells. J Microsc. 1997;186:84-7.

55. Heraty J, Hawks D. Hexamethyldisilazane - A chemical alternative for drying insects. Entomol News. 1998;109:369-74.

56. Laforsch C, Tollrian R. A new preparation technique of daphnids for Scanning Electron Microscopy using hexamethyldisilazane. Arch Hydrobiol. 2000;149:587-96.

57. Wichard W, Arens W, Eisenbeis G. Atlas zur Biologie der Wasserinsekten. Springer Spektrum; 1999. https://doi.org/10.1007/978-3-642-39452-2.

58. Pohl H. A scanning electron microscopy specimen holder for viewing different angles of a single specimen. Microsc Res Tech. 2010;73:1073-6.

59. Maugel T, Bonar D, Creegan W, Small E. Specimen preparation techniques for aquatic organisms. Scan Electron Microsc. 1980;2:51-71.

60. Suzuki T, Shibata M, Tanaka K, Tuchida K, Toda T. A new drying method, low-vacuum SEM freeze drying and its application to plankton observation. Bull Plankton Soc Japan. 1995::53-62.

61. Martin J, Olesen J, Hoeg J. Atlas of Crustacean Larvae. Maryland: JHU Press; 2014.

62. Rice A. Crab zoeal morphology and its bearing on the classification of the Brachyura. Trans Zool Soc Lond. 1980;35:271-424.

63. Ingle R. Larval stages of north-eastern Atlantic crabs: an illustrated key. London: Chapmann and Hall; 1992.

64. Williamson D. Larval morphology and diversity. In: Abele LG, editor. The Biology of Crustacea: 2 Embryology, morphology and genetics. New York: Academic press; 1982. p. 43-110.

65. Wohlfrom H, Melzer RR. Development of the sensory system in larvae and pupae of Chaoborus crystallinus (DeGeer, 1776; Diptera, Chaoboridae): sensory cells, nerves and ganglia of the tail region. Dev Genes Evol. 2001;211:124-37.

66. Meyer R, Martin J, Melzer RR. Nucleus patterns of zoea I larvae (Crustacea: Decapoda) in the context of taxonomy. Zootaxa. 2010;2422:31-42.

67. Veuthey T, Herrera G, Dodero VI. Dyes and stains: from molecular structure to histological application. Front Biosci. 2014;19:91-112.
68. Davie PJF, Guinot D, Ng PKL. Anatomy and functional morphology of Brachyura. In: Castro P, Davie P, Guinot D, Schram F, von Vaupel Klein C, editors. Treatise on Zoology - Anatomy, Taxonomy, Biology. The Crustacea, Volume 9 Part C (2 vols). BRILL; 2015. https://doi.org/10. 1163/9789004190832_004.

69. Felgenhauer BE. Internal anatomy of the Decapoda: an overview. In: Harrison FW, Humus AG, editors. Microscopic anatomy of invertebrates Vol :10. New York: Wiley-Liss; 1992. p. 45-75.

70. Holdich D. Biology of Freshwater Crayfish. Amsterdam: Wiley; 2001.

71. Bell TA, Lightner DV. A handbook of normal penaeid shrimp histology. Baton Rouge, LA (USA) World Aquaculture Society; 1988. https:// agris.fao.org/agris-search/search.do?recordID=XF2016018755.

72. Mulisch M, Welsch U. Romeis - Mikroskopische Technik. Springer. https://www.springer.com/de/book/9783642551895.

73. Hayat MA. Principles and techniques of electron microscopy: biological applications. 4th ed. Cambridge: Cambridge University Press; 2000

74. Keil TA. Morphology and development of the peripheral olfactory organs. In: Hansson BS, editor. Insect olfaction. Springer Berlin Heidelberg; 1999. p. 5-47. https://doi.org/10.1007/978-3-662-07911-9_2.

75. Keil TA. Sensory cilia in arthropods. Arthropod Struct Dev. 2012:41:515-34.

76. Keil TA, Steinbrecht RA. Mechanosensitive and olfactory sensilla of insects. In: King RC, Akai H, editors. Insect ultrastructure. Springer US; 1984. p. 477-516. https://doi.org/10.1007/978-1-4613-2715-8_13.

77. Geiselbrecht H, Melzer RR. How do mandibles sense? - The sensory apparatus of larval mandibles in Palaemon elegans Rathke, 1837 (Decapoda, Palaemonidae). Arthropod Struct Dev. 2013;42:1-16.

78. Geiselbrecht $H$, Melzer RR. Fine structure and ecdysis of mandibular sensilla associated with the lacinia mobilis in Neomysis integer (Leach, 1814) (Crustacea, Malacostraca, Peracarida). Arthropod Struct Dev. 2014:43:221-30.

79. Mclntosh R, Nicastro D, Mastronarde D. New views of cells in 3D: an introduction to electron tomography. Trends Cell Biol. 2005;15:43-51.

80. Höhn K, Sailer M, Wang L, Lorenz M, Schneider M, Walther P. Preparation of cryofixed cells for improved 3D ultrastructure with scanning transmission electron tomography. Histochem Cell Biol. 2011;135:1-9.

81. Steinbrecht RA, Zierold K, editors. Cryotechniques in biological electron microscopy. Berlin Heidelberg: Springer-Verlag; 1987. https://doi.org/10 1007/978-3-642-72815-0.

82. Lin J, Nicastro D. Asymmetric distribution and spatial switching of dynein activity generates ciliary motility. Science. 2018;360:eaar1968.

83. Steinbrecht RA. Freeze-substitution for morphological and immunocytochemical studies in insects. Microsc Res Tech. 1993;24:488-504.

84. Ruthensteiner B. Soft Part 3D visualization by serial sectioning and computer reconstruction. Zoosymposia. 2008;1:63-100.

85. Howard DW, Lewis EJ, Keller BJ, Smith CS. Histological techniques for marine bivalve mollusks and crustaceans. Oxford, MD: NOAA Technical Centers for Coastal Ocean Science; 2004. http://docs.lib.noaa.gov/ noaa_documents/NOS/NCCOS/TM_NOS_NCCOS/nos_nccos_5.pdf. Accessed 5 Feb 2021.

86. Lang G. Histotechnik - Praxislehrbuch für die Biomedizinische Analytik. Springer; 2013.

87. Vehof J, van der Meij S, Türkay M, Becker C. Female reproductive morphology of coral-inhabiting gall crabs (Crustacea: Decapoda: Brachyura: Cryptochiridae). Acta Zool. 2016;97:117-26.

88. Vehof J, Scholtz G, Becker C. Paradorippe granulata - A crab with external fertilization and a novel type of sperm storage organ challenges prevalent ideas on the evolution of reproduction in Eubrachyura (Crustacea: Brachyura: Dorippidae). Arthropod Struct Dev. 2018;47:82-90.

89. Kienbaum K, Scholtz G, Becker C. The morphology of the male and female reproductive system in two species of spider crabs (Decapoda: Brachyura: Majoidea) and the issue of the velum in majoid reproduction. Arthropod Systemat Phylo. 2017;75:245-60.

90. Kienbaum K, Vehof J, Becker C, Scholtz G. The reproductive system of Limnopilos naiyanetri indicates a thoracotreme affiliation of Hymenosomatidae (Decapoda, Eubrachyura). Arthropod Struct Dev. 2018:47:513-20. 
91. Suzuki Y, Imada T, Yamaguchi I, Yoshitake H, Sanada H, Kashiwagi T, et al. Effects of prolonged water washing of tissue samples fixed in formalin on histological staining. Biotech Histochem. 2012;87:241-8.

92. Holländer $\mathrm{H}$, Vaaland JL. A reliable staining method for semi-thin sections in experimental neuroanatomy. Brain Res. 1968;10:120-6.

93. Boshier D, Holloway H, Kitchin L. A comparison of standard lipid staining techniques used in electron microscopic studies of mammalian tissues. Stain Technol. 1984;59:83-9.

94. Ito S, Winchester RJ. The fine structure of the gastric mucosa in the bat. J Cell Biol. 1963;16:541-77.

95. Sridharan G, Shankar AA. Toluidine blue: a review of its chemistry and clinical utility. J Oral Maxillofac Pathol. 2012;16:251-5.

96. Glauert AM. Fixation. Dehydration and Embedding of Biological Specimens. Reprint. Amsterdam: Elsevier Science; 1984.

97. Pernstich A, Krenn HW, Pass G. Preparation of serial sections of arthropods using 2,2-dimethoxypropane dehydration and epoxy resin embedding under vacuum. Biotech Histochem. 2003;78:5-9.

98. Handschuh S, Baeumler N, Schwaha T, Ruthensteiner B. A correlative approach for combining microCT, light and transmission electron microscopy in a single 3D scenario. Front Zool. 2013;10:44.

99. Henne S, Friedrich F, Hammel JU, Sombke A, Schmidt-Rhaesa A. Reconstructing the anterior part of the nervous system of Gordius aquaticus (Nematomorpha, cycloneuralia) by a multimethodological approach. J Morphol. 2016;278:106-18.

100. Holst S, Michalik P, Noske M, Krieger J, Sötje I. Potential of X-ray micro-computed tomography for soft-bodied and gelatinous cnidarians with special emphasis on scyphozoan and cubozoan statoliths. $J$ Plankton Res. 2016;38:1225-42.

101. Silva MS, Carbayo F. X-ray microcomputed tomography applied to the taxonomic study of rare material: redescriptions of seven of Schirch's Brazilian species of land planarians (Geoplanidae, Platyhelminthes). Zookeys. 2020;910:1-42.

102. O'Sullivan JDB, Behnsen J, Starborg T, MacDonald AS, PhythianAdams AT, Else KJ, et al. X-ray micro-computed tomography $(\mu \mathrm{CT})$ : an emerging opportunity in parasite imaging. Parasitology. 2018;145:848-54

103. Dinley J, Hawkins L, Paterson G, Ball A, Sinclair I, Sinnett-Jones P, et al. Micro-computed $X$-ray tomography: a new non-destructive method of assessing sectional, fly-through and 3D imaging of a soft-bodied marine worm. J Microsc. 2010;238:123-33.

104. Faulwetter S, Vasileiadou A, Kouratoras M, Dailianis T, Arvanitidis C. Micro-computed tomography: Introducing new dimensions to taxonomy. ZK. 2013;263:1-45.

105. Sumner-Rooney L, Kenny NJ, Ahmed F, Williams ST. The utility of micro-computed tomography for the non-destructive study of eye microstructure in snails. Sci Rep. 2019;9:1-9.

106. Ziegler A. Combined visualization of echinoderm hard and soft parts using contrast-enhanced micro-computed tomography. Zoosymposia. 2019;15:172-91.

107. Ferstl S, Schwaha T, Ruthensteiner B, Hehn L, Allner S, Müller M, et al. Nanoscopic X-ray tomography for correlative microscopy of a small meiofaunal sea-cucumber. Sci Rep. 2020;10:1-12.

108. Michalik P, Piacentini L, Lipke E, Ramirez M. The enigmatic Otway odd-clawed spider (Progradungula otwayensis Milledge, 1997, Gradungulidae, Araneae): Natural history, first description of the female and micro-computed tomography of the male palpal organ. ZooKeys. 2013;335:101-12.

109. Sombke A, Lipke E, Michalik P, Uhl G, Harzsch S. Potential and limitations of X-Ray micro-computed tomography in arthropod neuroanatomy: A methodological and comparative survey. J Comp Neurol. 2015;523:1281-95.

110. Akkari N, Enghoff $\mathrm{H}$, Metscher BD. A New Dimension in Documenting New Species: High-Detail Imaging for Myriapod Taxonomy and First 3D Cybertype of a New Millipede Species (Diplopoda, Julida, Julidae). PLOS ONE. 2015;10:e0135243.

111. Nischik ES, Krieger J. Evaluation of standard imaging techniques and volumetric preservation of nervous tissue in genetically identical offspring of the crayfish Procambarus fallax cf. virginalis (Marmorkrebs). PeerJ. 2018;6:e5181.
112. Küpper SC, Klass K-D, Uhl G, Eberhard MJB. Comparative morphology of the internal female genitalia in two species of Mantophasmatodea. Zoomorphology. 2019;138:73-83.

113. Steinhoff POM, Uhl G, Harzsch S, Sombke A. Visual pathways in the brain of the jumping spider Marpissa muscosa. J Comp Neurol. 2020;528(11):1883.

114. Huys R, Olesen JT, Petrunina A, Martin JW. Tantulocarida. In: Martin JW, Olesen JT, Høeg JT, editors. Atlas of Crustacean Larvae. Baltimore: Johns Hopkins University Press; 2014. p. 122-7.

115. Ahyong ST, Haug JT, Haug C. Stomatopoda. In: Martin JW, Olesen JT, Høeg JT, editors. Atlas of Crustacean Larvae. Baltimore: Johns Hopkins University Press; 2014. p. 185-7.

116. Metscher BD. MicroCT for comparative morphology: simple staining methods allow high-contrast 3D imaging of diverse non-mineralized animal tissues. BMC Physiol. 2009;9:11.

117. Bushong EA, Johnson DD, Kim K-Y, Terada M, Hatori M, Peltier ST, et al. $X$-Ray microscopy as an approach to increasing accuracy and efficiency of serial block-face imaging for correlated light and electron microscopy of biological specimens. Microsc Microanal. 2015;21:231-8.

118. Dederichs TM, Müller CHG, Sentenská L, Lipke E, Uhl G, Michalik P. The innervation of the male copulatory organ of spiders (Araneae) - a comparative analysis. Front Zool. 2019;16:39.

119. Köhnk S, Baudewig J, Brandis D, Boretius S. What's in this crab? MRI providing high-resolution three-dimensional insights into recent finds and historical collections of Brachyura. Zoology. 2017;121:1-9.

120. Hounsfield GN. Computerized transverse axial scanning (tomography): Part 1. Description of system BJR. 1973:46:1016-22.

121. Rühr PT, Lambertz M. Surface contrast enhancement of integumentary structures in X-ray tomography. J Anat. 2019;235:379-85.

122. Krieger J, Spitzner F. X-Ray Microscopy of the Larval Crustacean Brain. In: Sprecher SG, editor. Brain Development: Methods and Protocols. New York, NY: Springer; 2020. p. 253-70. https://doi.org/10.1007/978-1-49399732-9_14.

123. Lehmann T, Melzer RR. Also looking like Limulus? - retinula axons and visual neuropils of Amblypygi (whip spiders). Front Zool. 2018;15:52.

124. Dömel JS, Macher T-H, Dietz L, Duncan S, Mayer C, Rozenberg A, et al. Combining morphological and genomic evidence to resolve species diversity and study speciation processes of the Pallenopsis patagonica (Pycnogonida) species complex. Front Zool. 2019;16:36.

125. Hita-Garcia F, Lieberman Z, Audisio TL, Liu C, Economo EP. Revision of the highly specialized ant genus Discothyrea (Hymenoptera: Formicidae) in the Afrotropics with X-ray microtomography and 3D cybertaxonomy. Insect Syst Divers. 2019. https://doi.org/10.1093/isd/ixz015.

126. Hörnig MK, Sombke A, Haug C, Harzsch S, Haug JT. What nymphal morphology can tell us about parental investment - a group of cockroach hatchlings in Baltic Amber documented by a multi-method approach. Palaeontol Electron. 2016:19:1-20.

127. Pohl H, Hammel JU, Richter A, Beutel RG. The first-fossil free living late instar larva of Strepsiptera Insecta. Arthropod Syst Hylogeny. 2019;77:125-40.

128. Liu Y, Melzer RR, Haug JT, Haug C, Briggs DEG, Hörnig MK, et al. Threedimensionally preserved minute larva of a great-appendage arthropod from the early Cambrian Chengjiang biota. Proc Natl Acad Sci U S A. 2016;113:5542-6.

129. Serrano-Sánchez M de L, Nagler C, Haug C, Haug JT, Centeno-García E, Vega FJ. The first fossil record of larval stages of parasitic isopods: cryptoniscus larvae preserved in Miocene amber. Neues Jahrbuch für Geologie und Paläontologie - Abhandlungen. 2016;279:97-106.

130. Broly P, Serrano-Sánchez M de L, Rodríguez-García S, Vega FJ. Fossil evidence of extended brood care in new Miocene Peracarida (Crustacea) from Mexico. Journal of Systematic Palaeontology. 2017;15:1037-49.

131. Schädel M, Perrichot V, Haug JT. Exceptionally preserved cryptoniscium larvae - morphological details of rare isopod crustaceans from French Cretaceous Vendean amber. Palaeontol Electron. 2019;22:1-46.

132. Bucher D, Scholz M, Stetter M, Obermayer K, Pflüger H-J. Correction methods for three-dimensional reconstructions from confocal images: I. tissue shrinking and axial scaling. J Neurosci Methods. 2000;100:135-43.

133. Beltz BS, Burd GD. Immunocytochemical techniques: Principles and practice. Cambridge, MA: Blackwell Scientific Publishing; 1989. 
134. Kalyuzhny AA. Immunohistochemistry - Essential Elements and Beyond. Springer Berlin Heidelberg. https://www.springer.com/de/book/97833 19308913. Accessed 5 Sep 2020

135. Cieluch U, Anger K, Aujoulat F, Buchholz F, Charmantier-Daures M, Charmantier $\mathrm{G}$. Ontogeny of osmoregulatory structures and functions in the green crab Carcinus maenas (Crustacea, Decapoda). J Exp Biol. 2004; 207:325-36

136. Cieluch U, Anger K, Charmantier-Daures M, Charmantier G. Osmoregulation and immunolocalization of $\mathrm{Na}+/ \mathrm{K}+$-ATPase during the ontogeny of the mitten crab Eriocheir sinensis (Decapoda, Grapsoidea). Mar Ecol Prog Ser. 2007;329:169-78.

137. Harzsch S, Dawirs RR. A developmental study of serotonin-immunoreactive neurons in the larval central nervous system of the spider crab Hyas araneus (Decapoda, Brachyura). Invertebrate Neurosci. 1995;1:53-65.

138. Harzsch S, Dawirs RR. Development of neurons exhibiting Fmrfamiderelated immunoreactivity in the central nervous system of larvae of the spider crab Hyas araneus L. (Decapoda: Majidae). J Crustacean Biol. 1996;16:10.

139. Beltz BS, Zhang Y, Benton JL, Sandeman DC. Adult neurogenesis in the decapod crustacean brain: a hematopoietic connection? Adult neurogenesis in the decapod crustacean brain. Eur J Neurosci. 2011;34:870-83.

140. Beltz BS, Brenneis G, Benton JL. Adult neurogenesis: lessons from Crayfish and the Elephant in the Room. Brain Behav Evol. 2016;87:146-55.

141. Beltz BS, Cockey EL, Li J, Platto JF, Ramos KA, Benton JL. Adult neural stem cells: long-term self-renewal, replenishment by the immune system, or both? BioEssays. 2015;37:495-501.

142. Brenneis G, Beltz BS. Adult neurogenesis in crayfish: Origin, expansion, and migration of neural progenitor lineages in a pseudostratified neuroepithelium. J Comp Neurol. 2020:528:1459-85.

143. Wittfoth C, Harzsch S. Adult neurogenesis in the central olfactory pathway of dendrobranchiate and caridean shrimps: new insights into the evolution of the deutocerebral proliferative system in reptant decapods. Dev Neurobiol. 2018;78:757-74.

144. Harzsch S, Miller J, Benton JL, Dawirs RR, Beltz BS. Neurogenesis in the thoracic neuromeres of two crustaceans with different types of metamorphic development. J Exp Biol. 1998;201:2465-79.

145. Harzsch S, Dawirs RR. Neurogenesis in larval stages of the spider crab Hyas araneus (Decapoda, Brachyura): proliferation of neuroblasts in the ventral nerve cord. Roux's Arch Dev Biol. 1994;204:93-100.

146. Sullivan JM, Macmillan DL. Embryonic and postembryonic neurogenesis in the ventral nerve cord of the freshwater crayfish Cherax destructor. J Exp Zool A Ecol Genet Physiol. 2001;290:49-60.

147. Harzsch S, Dawirs RR. Neurogenesis in the developing crab brain: postembryonic generation of neurons persists beyond metamorphosis. J Neurobiol. 1996:29:384-98.

148. Harzsch S, Miller J, Benton JL, Beltz BS. From embryo to adult: persistent neurogenesis and apoptotic cell death shape the lobster deutocerebrum. J Neurosci. 1999;19:3472-85.

149. Sintoni S, Benton JL, Beltz BS, Hansson BS, Harzsch S. Neurogenesis in the central olfactory pathway of adult decapod crustaceans: development of the neurogenic niche in the brains of procambarid crayfish. Neural Dev. 2012;7:1.

150. Harzsch S, Dawirs RR. Maturation of the compound eyes and eyestalk ganglia during larval development of the brachyuran crustaceans Hyas araneus L. (Decapoda, Majidae) and Carcinus maenas L. (Decapoda, Portunidae). Zoology. 1995:99:189-204.

151. Wildt M, Harzsch S. A new look at an old visual system: structure and development of the compound eyes and optic ganglia of the brine shrimp Artemia salina Linnaeus, 1758 (Branchiopoda, Anostraca). J Neurobiol. 2002;52:117-32.

152. Harzsch S, Benton J, Dawirs RR, Beltz B. A new look at embryonic development of the visual system in decapod crustaceans: Neuropil formation, neurogenesis, and apoptotic cell death. J Neurobiol. 1999;39:294-306

153. Schindelin J, Arganda-Carreras I, Frise E, Kaynig V, Longair M, Pietzsch T, et al. Fiji: an open-source platform for biological-image analysis. Nat Methods. 2012:9:676-82.

154. Altman JS, Tyrer NM. Filling selected neurons with cobalt through cut axons. In: Neuroanatomical Techniques. (Strausfeld NJ, Miller TA eds.). Springer Berlin Heidelberg; 1980. p. 373-402.

155. Friedrich M, Rambold I, Melzer RR. The early stages of ommatidial development in the flour beetle Tribolium castaneum (Coleoptera; Tenebrionidae). Dev Genes Evol. 1996;206:136-46.

156. Konno A, Okazaki S. Aqueous-based tissue clearing in crustaceans. Zool Lett. 2018:4:13.

157. GIMP. GIMP. https://www.gimp.org/. Accessed 5 Feb 2021.

158. MeeSoft. https://meesoft.com/. Accessed 5 Feb 2021.

159. Limaye A. Drishti: a volume exploration and presentation tool. In: Stock SR, editor. San Diego, California, USA; 2012. p. 85060X. https://doi.org/ $10.1117 / 12.935640$

160. Sutton MD, Garwood RJ, Siveter DJ, Siveter DJ. SPIERS and VAXML: A software toolkit for tomographic visualisation and a format for virtual specimen interchange. Palaeontol Electron. 2012;15:1-14.

161. Belevich I, Joensuu M, Kumar D, Vihinen H, Jokitalo E. Microscopy image browser: a platform for segmentation and analysis of multidimensional datasets. PLoS Biol. 2016;14:e1002340.

162. Foundation B. blender.org - Home of the Blender project - Free and Open 3D Creation Software. blender.org. https://www.blender.org/. Accessed 5 Feb 2021

163. Rötzer MAIN, Haug JT. Larval development of the European lobster and how small heterochronic shifts lead to a more pronounced metamorphosis. Int J Zool. 2015. https://doi.org/10.1155/2015/345172.

164. Rudolf NR, Haug C, Haug JT. Functional morphology of giant mole crab larvae: a possible case of defensive enrollment. Zool Lett. 2016:2:17.

165. Gundi P, Cecchin C, Fetzer L-L, Haug C, Melzer RR, Haug JT. Giant planktic larvae of anomalan crustaceans and their unusual compound eyes. Helgoland Mar Res. 2020;74:8.

166. Meyer R, Melzer R. Schwebende Ritter im Meerwasseraquarium. DATZ. 2006;59:12-4

167. Geiselbrecht H, Melzer RR. Nervous systems in 3D: A comparison of Caridean, Anomuran, and Brachyuran zoea-I (Decapoda). J Exp Zool B Mol Dev Evol. 2013;320:511-24.

\section{Publisher's Note}

Springer Nature remains neutral with regard to jurisdictional claims in published maps and institutional affiliations. 